\title{
A Review on the Synthesis, Characterization, and Modeling of Polymer Grafting
}

\author{
Miguel Ángel Vega-Hernández ${ }^{1}$, Gema Susana Cano-Díaz ${ }^{1}$, Eduardo Vivaldo-Lima 1,2,*(D), \\ Alberto Rosas-Aburto ${ }^{1}$, Martín G. Hernández-Luna ${ }^{1}$, Alfredo Martinez ${ }^{3}$, Joaquín Palacios-Alquisira ${ }^{4}$, \\ Yousef Mohammadi ${ }^{5}$ and Alexander Penlidis ${ }^{2, *(\mathbb{D})}$
}

check for updates

Citation: Vega-Hernández, M.Á.; Cano-Díaz, G.S.; Vivaldo-Lima, E.; Rosas-Aburto, A.; Hernández-Luna, M.G.; Martinez, A.;

Palacios-Alquisira, J.; Mohammadi, Y.; Penlidis, A. A Review on the Synthesis, Characterization, and Modeling of Polymer Grafting.

Processes 2021, 9, 375.

https://doi.org/10.3390/pr9020375

Academic Editor: Selestina Gorgieva

Received: 4 December 2020

Accepted: 26 January 2021

Published: 18 February 2021

Publisher's Note: MDPI stays neutral with regard to jurisdictional claims in published maps and institutional affiliations.

Copyright: (C) 2021 by the authors. Licensee MDPI, Basel, Switzerland. This article is an open access article distributed under the terms and conditions of the Creative Commons Attribution (CC BY) license (https:/ / creativecommons.org/licenses/by/ $4.0 /)$.
1 Departamento de Ingeniería Química, Facultad de Química, Universidad Nacional Autónoma de México, Ciudad de México 04510, Mexico; angeluz_alchemist@hotmail.com (M.Á.V.-H.); suscad@unam.mx (G.S.C.-D.); alberto_rosas_aburto@comunidad.unam.mx (A.R.-A.); martinhl@unam.mx (M.G.H.-L.)

2 Department of Chemical Engineering, Institute for Polymer Research, University of Waterloo, Waterloo, ON N2L 3G1, Canada

3 Instituto de Biotecnología, Universidad Nacional Autónoma de México, Cuernavaca, Morelos 62210, Mexico; alfredo.martinez@mail.ibt.unam.mx

4 Departamento de Fisicoquímica, Facultad de Química, Universidad Nacional Autónoma de México, Ciudad de México 04510, Mexico; polylab1@unam.mx

5 Petrochemical Research and Technology Company (NPC-rt), National Petrochemical Company (NPC), Tehran P.O. Box 14358-84711, Iran; mohammadi@npc-rt.ir

* Correspondence: vivaldo@unam.mx (E.V.-L.); penlidis@uwaterloo.ca (A.P.); Tel.: +519-888-4567 (ext. 36634) (A.P.)

Abstract: A critical review on the synthesis, characterization, and modeling of polymer grafting is presented. Although the motivation stemmed from grafting synthetic polymers onto lignocellulosic biopolymers, a comprehensive overview is also provided on the chemical grafting, characterization, and processing of grafted materials of different types, including synthetic backbones. Although polymer grafting has been studied for many decades - and so has the modeling of polymer branching and crosslinking for that matter, thereby reaching a good level of understanding in order to describe existing branching/crosslinking systems-polymer grafting has remained behind in modeling efforts. Areas of opportunity for further study are suggested within this review.

Keywords: polymer grafting; polymer synthesis; polymer characterization; mathematical modeling; polymer reaction engineering; reversible deactivation radical polymerization

\section{Introduction}

Graft copolymers consist of branches of polymer segments covalently bonded to primary polymer chains. Graft copolymers containing a single branch are known as miktoarm star copolymers. The backbone and branches can be homo- or copolymers with different chemical structures or compositions [1]. However, if the polymer molecule is a homopolymer, the reaction route to produce the branches is known as polymer branching; polymer grafting is usually considered as a chemical route to produce materials whose branches are chemically different from the backbone or primary polymer chain. The branches typically have the same chain size and are randomly distributed throughout the backbone's length as a consequence of the synthetic route used synthesize them. However, more efficient methods that allow the synthesis of graft copolymers with equidistant and same-length branches, with which the microstructure and composition can be controlled to a remarkable level, have been developed [1]. From a surface-chemistry perspective, this definition of polymer grafting is extended to composites in which the main chain constitutes a diverse array of materials, ranging from brick and fiberglass to paper and wood [2]. Materials with improved or simply different polymer properties from mechanical, thermal, melt flow or dilute solution perspectives can be synthesized by polymer 
grafting [1,3-7]. The structure-properties relationship has been an important issue in the analysis of polymer grafting [1].

Some of the first reports on polymer grafting available in the open literature (e.g., the oldest records available through Web of Science) include the grafting of polystyrene (PSty) [8] and poly(methyl methacrylate) (PMMA) [9] onto "government rubber styrene" (GRS) [8], which is a synthetic copolymer of butadiene and styrene, or onto natural rubber [9]; grafting of PSty, poly(butyl methacrylate) (PBMA), poly(lauryl methacrylate) (PLMA), poly(methyl acrylate) (PMA), and poly(ethyl acrylate) (PEA) onto PMMA with pendant mercaptan groups [10]; grafting of polyacrylamide (PAM) onto polyacrylonitrile (PAN), or the other way around (PAN onto PAM) [11]; grafting of PMMA onto PAN [12]; grafting of PSty onto polyethylene (PE) [13]; and grafting of several polymers, such as PAN, PMMA, PSty, poly(acrylic acid) (PAA), and poly(vinylidene chloride) (PVDC), onto cellulose [14-16], to name a few. A more complete literature review on the chemistry of polymer grafting is summarized in Table 1 . 
Table 1. Overview of the synthesis and characterization of grafted copolymers with an emphasis on the period 1950 to 1970 , plus some additional, more recent ones.

\begin{tabular}{|c|c|c|c|c|c|c|}
\hline Backbone & $\begin{array}{l}\text { Functionalization } \\
\text { Method }\end{array}$ & Graft Chains & $\begin{array}{l}\text { Grafting } \\
\text { Technique }\end{array}$ & Grafting Conditions & $\begin{array}{l}\text { Measured Properties and Characterization } \\
\text { Methods }\end{array}$ & Ref. \\
\hline $\begin{array}{l}\text { Rubber (GRS or } \\
\text { natural) }\end{array}$ & $\begin{array}{l}\text { Generation of internal } \\
\text { free radicals by CTP. }\end{array}$ & PSty or PMMA & Grafting from & $\begin{array}{l}95-180{ }^{\circ} \mathrm{C} \text {; Mass FRP (rubber dissolved in } \\
\text { monomer, in presence of initiator); } \\
\text { solvent-non-solvent fractionation. }\end{array}$ & $\begin{array}{l}\text { Determination of vinyl unsaturation (peracid } \\
\text { and infrared methods); molecular weight by } \\
\text { intrinsic viscosity; DMA; mechanical } \\
\text { properties: tensile strength, } \\
\text { elongation-at-break, hardness, modulus at } \\
100 \text { and } 300 \% \text { elongation, as well as tear at } 20 \text {, } \\
80 \text {, and } 120^{\circ} \mathrm{C} \text {. }\end{array}$ & {$[8,9]$} \\
\hline $\begin{array}{l}\text { PMMA (a } \\
\text { copolymer of } \\
\text { MMA and small } \\
\text { content of GMA) }\end{array}$ & $\begin{array}{l}\text { Incorporation of } \\
\text { mercaptan groups by } \\
\text { reaction of GMA and } \\
\text { hydrogen sulfide in } \\
\text { presence of sodium } \\
\text { ethoxide catalyst. }\end{array}$ & $\begin{array}{l}\text { PSty, PBMA, } \\
\text { PLMA, or PMA }\end{array}$ & Grafting from & $\begin{array}{l}\text { Mass or solution polymerization of } \\
\text { monomer in presence of PMMA with } \\
\text { pendant mercaptan groups (grafting } \\
\text { occurs by chain transfer to mercaptan } \\
\text { groups). }\end{array}$ & $\begin{array}{l}\text { Solvent extraction of ungrafted polymer, } \\
\text { measured by UV analysis; grafting efficiency } \\
\text { calculated with the aid of a kinetic model. }\end{array}$ & [10] \\
\hline Either PAN or PAM & $\begin{array}{l}\text { Two methods used: (a) } \\
\text { CTP; (b) photolysis of a } \\
\text { copolymer containing } \\
\text { a few per cent of ACN. }\end{array}$ & $\begin{array}{l}\text { PAM, PAN; } \\
\text { PMMA (onto } \\
\text { PAN) [12] }\end{array}$ & Grafting from & $\begin{array}{l}\text { (a) SP in sodium perchlorate at } 55^{\circ} \mathrm{C} \text {, } \\
\text { using persulphate-bisulphite; (b) SP in } \\
\text { sodium perchlorate at } 25 \text { to } 35^{\circ} \mathrm{C} \text { in a } \\
\text { quartz tube under a G.E. Sun Lamp. }\end{array}$ & $\begin{array}{l}\text { Composition by IR; molecular weight by } \\
\text { intrinsic viscosity calibrated from light } \\
\text { scattering data; phase contrast microscopy; } \\
\text { measurement of softening points; analysis of } \\
\text { X-ray scattering curves [12]. }\end{array}$ & {$[11,12]$} \\
\hline PE & $\begin{array}{l}\text { UV irradiation of } \\
\text { surface of sensitized } \\
\text { PE. }\end{array}$ & PSty & Grafting from & $\begin{array}{l}\text { (a) Sensitized PE irradiated one minute, } \\
\text { stand a week, and then proceed to mass } \\
\text { polymerization in Sty at } 70{ }^{\circ} \mathrm{C} \text {; (b) } \\
\text { irradiation of plastic in presence of Sty. }\end{array}$ & $\begin{array}{l}\text { Mass difference or ability of the surface to } \\
\text { adhere to pressure-sensitive tape under load. }\end{array}$ & [13] \\
\hline Cellulose & $\begin{array}{l}\gamma \text {-ray pre-irradiation } \\
\text { technique. }\end{array}$ & PSty & Grafting from & $\begin{array}{l}\text { Pre-irradiation by } \mathrm{Co}^{60} \gamma \text {-rays in water or } \\
\text { in a } \mathrm{H}_{2} \mathrm{O}_{2} \text { solution; grafting in a } 20 \text { vol. } \\
\text { Sty solution (methanol/water) at } 50^{\circ} \mathrm{C} \text {. }\end{array}$ & $\begin{array}{l}\text { Degree of grafting by weight gain; estimation } \\
\text { of active sites by the ferrous ion method; } \\
\text { solubilization of material by acetylation and } \\
\text { acetolysis, followed by IR spectroscopy. }\end{array}$ & [14] \\
\hline Cellulose & $\begin{array}{l}\text { Binding initiators or } \\
\text { components of } \\
\text { initiation systems by } \\
\text { means of ion exchange } \\
\text { with such materials. }\end{array}$ & $\begin{array}{l}\text { Various polymers } \\
\text { (PAN, PMMA, } \\
\text { PSty, PAA, } \\
\text { PVDC) }\end{array}$ & Grafting from & $\begin{array}{l}\text { Starting material contacted with a dilute } \\
\text { solution of catalyst cation salt; the } \\
\text { exchanged cellulose was then placed in } \\
\text { the monomer or monomer solution; the } \\
\text { mixture was heated or irradiated for the } \\
\text { required time. }\end{array}$ & $\begin{array}{l}\text { Swelling by centrifuging; grafting efficiency } \\
\text { by solvent extraction; mechanical properties } \\
\text { (elongation, moduli; toughness); chemical } \\
\text { properties (basic/acid dying and hydrolysis, } \\
\text { bromination, oxidation, complex formation, } \\
\text { etc.); wetting; rotproofing. }\end{array}$ & [15] \\
\hline
\end{tabular}


Table 1. Cont.

\begin{tabular}{|c|c|c|c|c|c|c|}
\hline Backbone & $\begin{array}{l}\text { Functionalization } \\
\text { Method }\end{array}$ & Graft Chains & $\begin{array}{l}\text { Grafting } \\
\text { Technique }\end{array}$ & Grafting Conditions & $\begin{array}{l}\text { Measured Properties and Characterization } \\
\text { Methods }\end{array}$ & Ref. \\
\hline Cellulose & $\begin{array}{l}\text { Formation of free } \\
\text { radicals in cellulose by } \\
\text { exposure to high } \\
\text { energy electrons or to } \\
\gamma \text {-rays from } \mathrm{Co}^{60} \text {. }\end{array}$ & PSty & Grafting from & $\begin{array}{l}\text { Sty brought into intimate contact with } \\
\text { cellulose by the inclusion technique; then, } \\
\text { (a) perform irradiation with high energy } \\
\text { electrons using a 2-M.e.v. Van de Graaff } \\
\text { accelerator; or (b) induce grafting by } \\
\gamma \text {-ray irradiation. }\end{array}$ & $\begin{array}{l}\text { Degree of grating and grafting efficiency } \\
\text { from extraction curves (mass determination); } \\
\text { drastic hydrolysis of cellulose backbone and } \\
\text { molecular weight determination of isolated } \\
\text { PSty chains by intrinsic viscosity; calculation } \\
\text { of grated chains per cellulose chain. }\end{array}$ & [16] \\
\hline $\begin{array}{l}\text { Terpolymer of } \\
\text { ethylene- ethyl } \\
\text { acrylate-maleic } \\
\text { anhydride } \\
\text { (EEAMA) }\end{array}$ & $\begin{array}{l}\text { Step-growth } \\
\text { polymerization (SGP) } \\
\text { between maleic } \\
\text { anhydride (MAnh) } \\
\text { from EEAMA and } \\
\text { amine groups from } \\
\text { PDMS. }\end{array}$ & $\begin{array}{l}\text { Polydi-methyl- } \\
\text { siloxane } \\
\text { (PDMS) }\end{array}$ & Grafting to & $\begin{array}{l}\text { Melt reactive mixing proceeded in a } \\
\text { Haake Rheocord } 3000 \text { batch mixer; } \\
\mathrm{T}=140{ }^{\circ} \mathrm{C} \text {. }\end{array}$ & $\begin{array}{l}\text { Composition and evidence of grafting by }{ }^{1} \mathrm{H} \\
\text { and }{ }^{13} \mathrm{C} \text { nuclear magnetic resonance. } \\
\text { Molecular weight distributions (MWDs) } \\
\text { were determined using a GPC or SEC } \\
\text { chromatograph (Alliance GPCV 2000, Waters) } \\
\text { with refractive index viscometer detectors. } \\
\text { Linear viscoelastic properties were } \\
\text { determined using a rheometer (AR 2000, TA } \\
\text { Instrument) with a cone-and-plate } \\
\text { configuration. }\end{array}$ & [17] \\
\hline
\end{tabular}

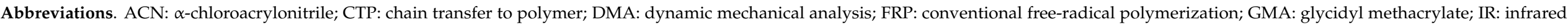

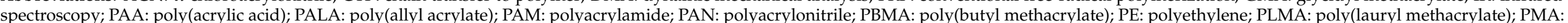
poly(methyl acrylate); PMMA: poly(methyl methacrylate); PSty: polystyrene; PVDC: poly(vinylidene chloride); REX: reactive extrusion; SP: solution polymerization. 
The renewed emphasis on the use of biobased monomers and biopolymers as a viable route to decrease (synthetic) polymer waste and disposal issues has invigorated the research efforts on the development of improved materials with important contents of biopolymers (frequently as backbones); grating is part of the synthetic procedure of such materials. These trends are in the scope of some recent review papers focused on polymer grafting, which include the grafting of polymers onto cellulose $[27,28]$, chitin/chitosan $[29,30]$, or polysaccharides in general [2,31].

The use of lignocellulosic waste as raw material for biorefining processes aimed at producing value-added chemicals or materials (e.g., bioethanol, cellulose, xylose, or hybrid materials, to name a few) has increased significantly since the start of the present century. Biorefineries from lignocellulosic waste require multistep processes, starting with pretreatment of the biomass. In this way, the constituent biopolymers are available for subsequent reactive processes [32-35]. The synthesis of value-added materials from lignocellulosic waste biomasses by using polymer grafting onto lignocellulose itself [36] or onto its individual components (cellulose [27], hemicellulose [37], or lignin [38]) represents an important route in the concept of biorefineries.

Although a few early studies focused on the mathematical descriptions of polymer grafting under specific circumstances-such as the calculation of grafting efficiency and molecular weight development of the grafted branches onto a pre-formed polymer containing pendant mercaptan groups capable of acting as effective chain transfer agents, based on a comprehensive kinetic model including chain transfer to polymer and bimolecular polymer radical termination [39], or the theoretical calculation of molecular weight distributions of vinyl polymers grafted onto solid polymeric substrates by irradiation, also based on a kinetic description of the growing of the grafted branches [40], and a few comprehensive recent models for other specific situations (e.g., the detailed description of free-radical polymerization (FRP)-induced branching in reactive extrusion of PE [41]) —are indeed available, the fact is that the cases addressed by mathematical models are by far less common than the available experimental systems. The purpose of the present review is to first offer a rather detailed summary of what is known from a polymer chemistry angle about polymer grafting, with an emphasis on what backbones and grafts are used, how active sites on backbones are generated, and how polymer branches are grown or grafted, among other process details. The second objective is to review what polymer grafting situations have been modeled, which tools have been used, and what limitations persist. By doing that, we can show areas of opportunity. Do keep in mind that the system that motivated this study was the grafting of synthetic polymers onto lignocellulosic biopolymers.

\section{Chemistry of Polymer Grafting}

The main chemical routes for polymer grafting are the following: "grafting onto" (also referred as "grafting to"), "grafting from," and the macromonomer or macromer (or "grafting through") method $[1,2,27,28]$. There are general reviews focused on the synthesis of grafted copolymers [1,2]. The ranges of backbones and grafts, backbone activating methods, graft growing (polymerization) routes, characterization techniques, quantification methods of grafting and branching molar mass distributions, and applications are so vast that reviews on specific aspects or subtopics related to these issues have been written. For instance, there are reviews focused on the grafting of polymer branches onto natural polymers [42] and biofibers [43]; grafting onto cellulose [27] or cellulose nanocrystals [28]; grafting onto chitin/chitosan [29,30]; microwave-activated grafting [31,44]; laccase-mediated grafting onto biopolymers and synthetic polymers [2]; radiation-induced RAFT-mediated graft copolymerization [45]; and polymer grafting onto inorganic nanoparticles [46] to name a few.

Herein, brief descriptions of such chemical routes are provided. In Table 1 we provide an overview of the grafted copolymer materials synthesized in the 1950-1970 period, plus some additional more recent cases, considering backbone structure, functionalization or active site generation techniques or procedures, grafted arm structure, polymer grafting 
technique, polymer grafting conditions, measured properties and characterization methods, and related references. The literature on polymer grafting onto cellulose, chitin/chitosan, lignocellulosic biopolymers, other polysaccharides and natural biopolymers, inorganic materials, and metallic surfaces is addressed in the subsequent sections of this review.

\subsection{Types of Polymer Grafting}

As stated earlier, polymer grafting can proceed by the "grafting to" technique, where a polymer molecule with a reactive end group reacts with the functional groups present in the backbone; by "grafting from," where polymer chains are formed from initiating sites within the backbone; and by "grafting through," where a macromolecule with a reactive end group copolymerizes with a second monomer of low molecular weight. Simplified representations of these grafting techniques are shown in Figure 1.

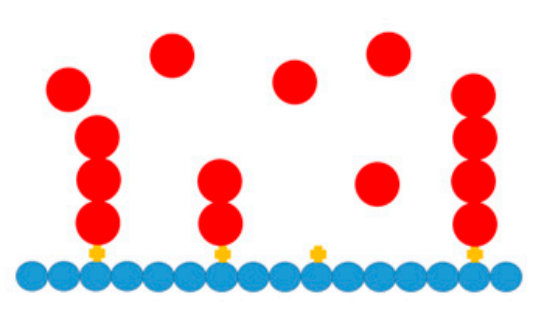

GRAFTING FROM

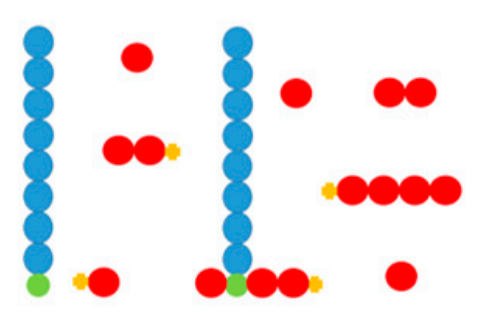

GRAFTING THROUGH

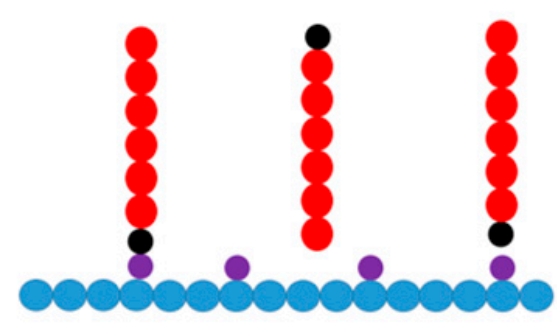

GRAFTING TO

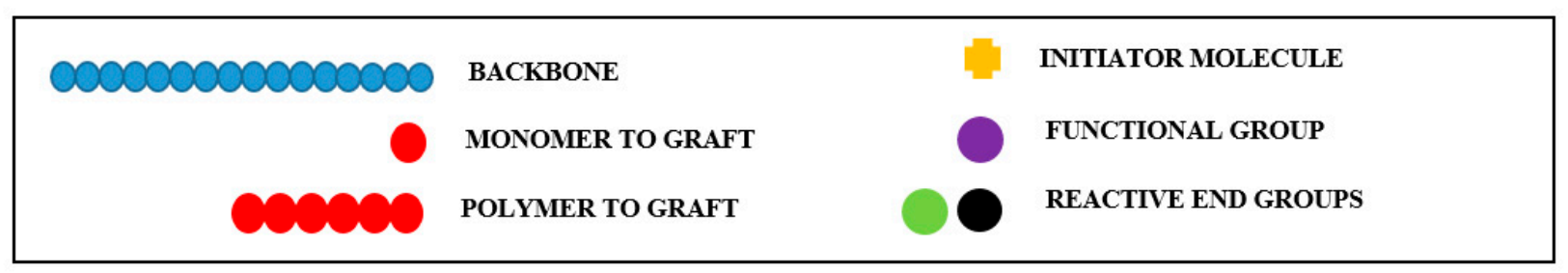

Figure 1. Polymer grafting chemical routes.

"Grafting to" and "grafting from" are the most common polymer grafting chemical routes. Better defined graft segments are obtained by the "grafting to" technique since the polymerization is independent of the union between the backbone and grafts. In contrast, materials of higher grafting densities can be produced by the "grafting from" route due to the lack of steric hindrance restrictions [47].

However, each polymer grafting route has its own advantages and disadvantages in terms of chemical nature, density, dispersity, and length of the grafts obtained, and the ease and efficiency of the chemical reactions involved. Interestingly, different polymer grafting routes can be combined to produce specific grafted materials [48].

\subsection{Main Backbones Used in Polymer Grafting}

A polymer backbone is a polymer molecule that supports polymeric side chains, called branches or grafts. Side chains can be inserted onto the backbone during the synthesis of the backbone (copolymerization situation) or as a post-production process of the backbone [49]. In the first case, polymers with homogeneous bulk properties are obtained. The second case is very attractive since it allows the modification of many polymeric materials, including natural and synthetic fibers, or inorganic and metal particles. Backbones processed by polymer modification do not usually show significant changes in bulk properties. Surface modification is often carried out following a "grafting from" technique; that is why this method is also known as surface initiated polymerization (SIP) [50]. The backbones used for polymer grafting can be synthetic polymers, biopolymers, or inorganic and metal surfaces. 
Synthetic polymers are human-made polymers and include a wide variety of materials, such as polyolefins, vinyl and fluorinated polymers, nylons, etc. The applications of synthetic graft copolymers include the synthesis of antifouling membranes [51], stimuliresponse materials [52], and biomedical applications [53].

Biopolymers are produced by the cells of living organisms. Polysaccharides have become important lately because of their characteristics of availability, biocompatibility, low cost, and non-toxicity, making them candidates for substitution of petroleum-based materials [47]. Polysaccharide-based graft copolymers are used as drug delivery carrier, food packaging and wastewater treatment [54]. Some of the most studied polysaccharides are cellulose [27,28], lignin [55], chitin/chitosan [29,56-58], starch [59], and various gums [42].

Surface functionalization of inorganic and metallic particles that allow the incorporation of polymer shells by polymer grafting has also become important, since polymer coatings alter the interfacial properties of the modified particles. Zhou et al. reviewed different applications for inorganic and metallic particles grafted with biopolymers [50]. One important inorganic surface modified by polymer grafting is silica [60].

\subsection{Backbone Functionalization Methods}

Several chemical modification procedures have been developed due to the wide variety of backbones of interest. Chemical modification reactions depend on the functional groups (or absence thereof) along the backbone. Two main chemical routes used to attach, grow, or graft polymer molecules onto lignin have been proposed [55]: (a) creation of new chemically active sites, and (b) functionalization of hydroxyl groups.

The introduction of functional groups into a polymer backbone increases its reactivity, making it accessible for forward polymerization or coupling reactions. Functionalization reactions are therefore required to generate the end functional pre-formed polymer or the reactive end of the macromolecule species involved in the "grafting to" and "grafting through" polymer grafting techniques, respectively. Functionalization is also required in the formation of the macromolecular species, such as macro-initiators and macro-controllers, involved in the "grafting from" polymer grafting technique [27,61]. The most important functionalization reactions involved in polymer grafting include sulfonation, esterification, etherification, amination, phosphorylation, and thiocarbonation, among others.

\subsection{Backbone Activation Methods}

Another way to generate grafting sites within the polymer backbone is to use polymer grafting activators, such as free-radical initiators. As shown in Figure 2, polymer grafting activators can be classified into physical, chemical, and biological. The main characteristics of these activators are highlighted in Sections 2.4.1-2.4.4. 


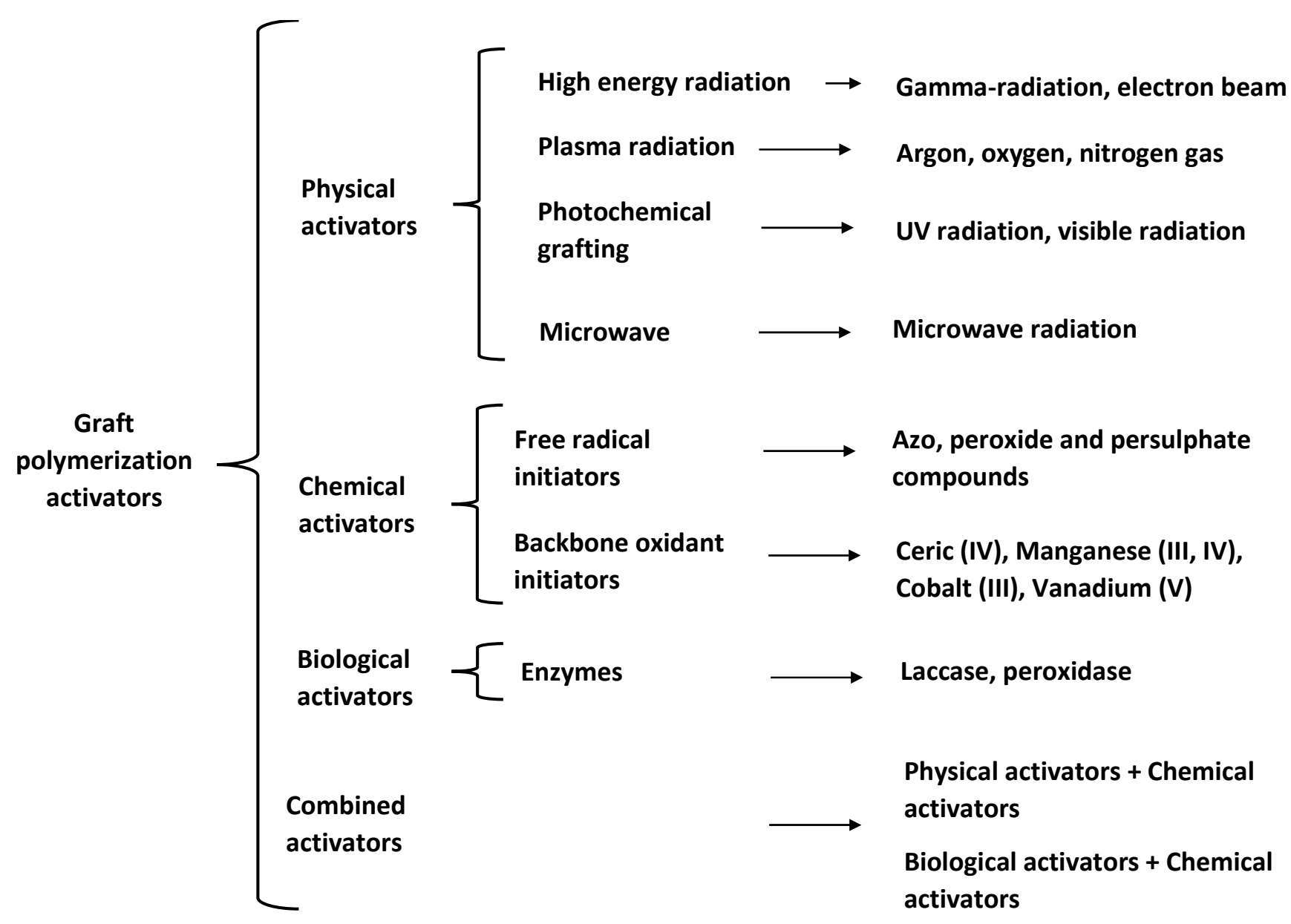

Figure 2. Backbone activators used for polymer grafting.

\subsubsection{Physical Activators}

High energy radiation, also referred to as ionizing radiation, includes $\gamma$-beam and electron-beam radiations. Radiation-promoted grafting may follow one of three possible routes: (a) pre-irradiation of the backbone in the presence of an inert gas to generate free radicals before placing the backbone in contact with monomers; (b) pre-irradiation of the backbone in an environment containing air or oxygen to produce hydroperoxides or diperoxides in its surface, followed by high temperature reaction with monomer; and (c) the mutual irradiation technique, where backbone and monomer are irradiated simultaneously to generate free radicals [62].

Plasma is a partially ionized gas where free electrons, ions, and radicals are mixed. Different functional groups can be introduced, or free radicals can be generated on backbones by this process, depending on the gas used. Polymer grafting reactions carried out in plasma are sometimes classified as high energy radiation reactions [63].

The absorption of UV light on the surface of the material generates free radicals that serve as nucleation sites. The surface is then placed in contact with monomer for subsequent polymerization [64].

Microwave irradiation consists of direct interaction of electromagnetic irradiation with polar molecules and ionic particles, promoting very fast non-contact internal heating, which enhances reaction rates and leads to higher yields. Singh et al. carried out a successful polymerization of acrylamide on guar gum under microwave irradiation [65]. They proposed a mechanism in which free radicals are produced within the polysaccharide backbone by the effect of microwave irradiation on the hydroxyl groups of the biopolymer [65]. 


\subsubsection{Chemical Activators}

As shown in Figure 1, chemical activators include free radical and backbone oxidant initiators. Free radical initiators are compounds that present either direct or indirect homolytic fission. The first case involves the initiator itself and the second one requires participation of another molecule from the environment [66].

Oxidant initiators react directly with functional groups from the backbone, generating activation sites. Polymer grafting of polysaccharides using oxidant initiators has been reported in the literature [66].

\subsubsection{Biological Activators}

Enzymes catalyze polymer modification reactions through functional groups located at chain ends, along the main chain, or at side branches, promoting highly specific nondestructive transformations on backbones, under mild reaction conditions. Successful grafting of lignin by oxidation of its phenolic structures using laccases has been reported recently [2].

\subsubsection{Combined Activators}

Combinations of physical and chemical activators for polymer grafting have been successfully carried out. For instance, microwave assisted polymerization (MAP) has been combined with the use of chemical activators for the production of hydrogels synthesized by crosslinking graft copolymerization, taking advantage of the short reaction times required to obtain high yields [67,68]. Enzymes are also used in combination with radical initiators for more effective grafting copolymerization processes $[69,70]$.

\subsection{Polymer Grafting by Free-Radical Polymerization}

As stated earlier, the "grafting through" and "grafting from" techniques require a polymerization reaction to bond the polymer grafts to the backbone. Different polymerization methods have been used for polymer grafting, but the most effective ones use free radical methods (e.g., FRP, RDRP, and REX), due to their versatility to work with different chemical groups, and their tolerance to impurities. A short overview on free-radical polymerization reactions is presented in Table 2. Polymer grafting by FRP, and other reactions, is affected by several factors, including the chemical nature of the components contained in the system - backbone, monomer, initiator, and solvent—and the interactions among them. Other aspects related to polymer grafting, including temperature and the use of additives, need to be considered [67]. The synthetic routes and activators used in graft polymerization provide a variety of interesting and versatile routes for this type of polymer modification.

Table 2. Free-radical polymerization methods.

\begin{tabular}{|c|c|c|}
\hline Polymerization Method & General Description & Type of Reaction \\
\hline $\begin{array}{l}\text { Conventional free-radical } \\
\text { polymerization (FRP) }\end{array}$ & $\begin{array}{l}\text { Three steps involved: (1) initiation, with formation of } \\
\text { free radicals; (2) propagation, where free radicals react } \\
\text { with monomer; and (3) termination of polymer radicals } \\
\text { by either combination, disproportionation, or chain } \\
\text { transfer to small molecules. The simultaneous } \\
\text { participation of these reactions leads to broad molar } \\
\text { mass distributions. }\end{array}$ & $\begin{array}{l}\text { Chain transfer reaction: free radicals generated in the } \\
\text { system tend to react with backbones by CTP, thereby } \\
\text { activating them. } \\
\text { Direct generation of free radicals along the backbone: } \\
\text { activators generate free radicals from reaction with } \\
\text { functional groups placed along the backbone, which } \\
\text { correspond to the initiating step of a SIP [27]. }\end{array}$ \\
\hline
\end{tabular}


Table 2. Cont.

\begin{tabular}{|c|c|c|}
\hline Polymerization Method & General Description & Type of Reaction \\
\hline $\begin{array}{l}\text { Reversible deactivation } \\
\text { radical polymerization } \\
\text { (RDRP) }\end{array}$ & $\begin{array}{l}\text { A group of polymerization techniques based on free } \\
\text { radical technology that controls the growth of polymer } \\
\text { molecules during the polymerization. } \\
\text { Polymer radicals are reversibly deactivated by effect of } \\
\text { controllers that act under some relatively new chemical } \\
\text { routes. These techniques allow the development of } \\
\text { advanced materials, with various architectures, and } \\
\text { well-defined microstructures. } \\
\text { Each technique has its own mechanism and conditions } \\
\text { that favor them. SIP can proceed by any of the known } \\
\text { RDRP techniques. }\end{array}$ & $\begin{array}{l}\text { Atom transfer radical polymerization (ATRP): It is a } \\
\text { catalytic process where an alkyl halide macromolecule } \\
\text { reacts with the catalyst, allowing the formation of a } \\
\text { radical that propagates until it reacts again with the } \\
\text { catalyst, in a reversible way [71]. } \\
\text { Nitroxide mediated polymerization (NMP): A stable } \\
\text { nitroxide free radical acts as controller, reversibly } \\
\text { deactivating the propagating and polymer radicals } \\
\text { forming dormant polymer molecules with } \\
\text { alkoxyamine end functionalities [50]. } \\
\text { Reversible addition-fragmentation chain transfer } \\
\text { (RAFT) polymerization: Thiocarbonilthio compounds } \\
\text { are used as chain transfer agents which control } \\
\text { molecular weight development by reversible } \\
\text { activation-deactivation reactions [50]. }\end{array}$ \\
\hline Reactive extrusion (REX) & $\begin{array}{l}\text { REX is a set of techniques designed to produce and } \\
\text { modify polymers, typically carried out in single or } \\
\text { twin extruders. Five main types of reactive } \\
\text { polymerizations caried out in extruders have been } \\
\text { reported: bulk polymerization, polymer grafting, } \\
\text { polymer functionalization, controlled degradation, and } \\
\text { reactive blending [72]. } \\
\text { Reactions proceed in melt phase. Examples of polymer } \\
\text { grafting by REX include polyolefin [73] and starch } \\
\text { modifications [74]. }\end{array}$ & $\begin{array}{l}\text { Polymer modification by free-radical polymerization: } \\
\text { Free radical initiators such as peroxides are used to } \\
\text { generate activate sites within the backbone }[73,75] \text {. } \\
\text { Polymer modification by insertion of active pendant } \\
\text { groups: It consists of the copolymerization of } \\
\text { monomers who have no functional groups with } \\
\text { co-monomers possessing pendant which make } \\
\text { polymer grafting easier to accomplish [49]. }\end{array}$ \\
\hline
\end{tabular}

\section{Backbones and Supports Used in Polymer Grafting}

As explained earlier, grafted materials consist of side chains or arms attached to primary polymers referred to as backbones. The purpose of polymer grafting is to combine chemical, mechanical, interfacial, electrical, or other polymer properties between the constituent materials. The diversity of backbones and the ways in which side chains are attached to them through polymer grafting will be briefly overviewed in this section.

\subsection{Cellulose, Lignin, and Lignocellulosic Biomasses as Backbones}

Lignocellulosic biopolymers are abundant in nature. They are made of cellulose, hemicellulose, and lignin. They also contain moisture, extractive organic compounds, and ashes from inorganic compounds in lesser amounts. Each of these components has distinct characteristics. The extractive organic compounds present in lignocellulosic biopolymers are oligomers and oligosaccharides of low molecular weight, sugars, fatty acids, resins, etc. [76].

Cellulose, hemicellulose, and lignin can be modified by polymer grafting leading to new promising materials with interesting properties. However, the extractables are not useful for this purpose since they are not part of a skeleton or stiff structure that may provide support or mechanical stability. Extractables also consume reactants required for the grafting process. They are usually removed prior to the polymer grafting process, although in some studies, they remain in the system during the formation of grafted arms [77].

Polymer grafting of xylan onto lignin has been studied since the early 1960s. Early reports on the topic reported the grafting of organic polymers, such as 4-methyl-2-oxy-3oxopent-4-ene and methyl methacrylate polymers [78,79], xylan [80], ethylbenzene, and styrene [81-84], onto lignin or lignin derivatives. The topic of polymer grafting of synthetic polymers onto lignocellulosic biopolymers has gained renewed relevance in the last two decades due to environmental and sustainability issues [27,85-94]. 
Table 3. Overview of grafting of synthetic polymers onto cellulose and natural fibers.

\begin{tabular}{|c|c|c|c|c|c|c|}
\hline Backbone & $\begin{array}{l}\text { Functionalization } \\
\text { Method }\end{array}$ & Grafted Chains & $\begin{array}{l}\text { Grafting } \\
\text { Technique }\end{array}$ & Grafting Conditions & Measured Properties and Methods & Refs. \\
\hline Cotton fabric & $\begin{array}{l}\text { FRP by a cellulose } \\
\text { thiocarbonate-AIBN } \\
\text { redox system. }\end{array}$ & $\begin{array}{l}\text { PMMA, PAA, } \\
\text { PAN, PAM }\end{array}$ & Grafting from & $\mathrm{T}=60-80{ }^{\circ} \mathrm{C}$ & $\begin{array}{l}\text { Degree of grafting (GP), gravimetric } \\
\text { method. }\end{array}$ & [95] \\
\hline Cellulose & $\begin{array}{l}\text { FRP by a KPS-FAS } \\
\text { REDOX system. }\end{array}$ & PSty, PAN & Grafting from & $\begin{array}{l}\mathrm{T}=60^{\circ} \mathrm{C} ; \mathrm{t}=3 \mathrm{~h} ;[\mathrm{STY}]=0.65 \mathrm{M} \\
{[\mathrm{KPS}]=0.14 \mathrm{M} ;[\mathrm{FAS}]=0.01 \mathrm{M}}\end{array}$ & $\begin{array}{l}\text { GP, gravimetric method; FTIR and TGA to } \\
\text { corroborate GP. }\end{array}$ & [96] \\
\hline Cellulose fabrics & FRP by KPS initiation. & PIA & Grafting from & $\begin{array}{l}\mathrm{T}=55-80^{\circ} \mathrm{C} ; \mathrm{t}=2.5-5 \mathrm{~h} ;[\mathrm{KPS}]= \\
0.05-0.5 \mathrm{M} ;[\mathrm{IA}]=0.5-4 \mathrm{M} .\end{array}$ & $\begin{array}{l}\text { GP, gravimetric method; FTIR, XRD, TGA } \\
\text { and SEM to corroborate grafting. }\end{array}$ & [97] \\
\hline Cellulose & FRP by CAAC initiation. & PMBA, P(N-VP) & Grafting from & $\mathrm{T}=30-70{ }^{\circ} \mathrm{C} ;[\mathrm{CAAC}]=2-30 \times 10^{-5} \mathrm{M}$ & $\begin{array}{l}\text { Grafting yield (GY) and other grafting } \\
\text { parameters; gravimetric method. }\end{array}$ & {$[98,99]$} \\
\hline Cellulose & $\begin{array}{l}\text { FRP by CAN-NAC } \\
\text { REDOX system. }\end{array}$ & $\begin{array}{l}\text { PEA, PNIPAAM, } \\
\text { PAAM-PEA, } \\
\text { (AAM-EMA), } \\
\text { P(AAM-MA), } \\
\text { P(AN-EMA) }\end{array}$ & Grafting from & $\begin{array}{l}\mathrm{T}=10-60^{\circ} \mathrm{C} ; \mathrm{t}=24 \mathrm{~h} ;[\mathrm{CAN}]=1.5-32 \times \\
10^{-3} \mathrm{M} ;[\mathrm{NAC}]=2.5-8 \times 10^{-2} \mathrm{M}\end{array}$ & $\begin{array}{l}\text { GY and other grafting parameters, } \\
\text { gravimetric method; FTIR and TGA to } \\
\text { corroborate grafting. FTIR and EA for } \\
\text { composition. MMass by viscometric } \\
\text { method and GPC. }\end{array}$ & [100-105] \\
\hline $\begin{array}{l}\text { Cellulose } \\
\text { microfibers }\end{array}$ & Redox initiation. & PAA & Grafting through & $\begin{array}{l}\text { KPS }=0.1-0.4 \% \text { respect to fiber weight. } \\
\mathrm{t}=3 \mathrm{~h}, \mathrm{~T}=75^{\circ} \mathrm{C}\end{array}$ & $\begin{array}{l}\text { GP, gravimetric method; FTIR and TGA to } \\
\text { corroborate GP. }\end{array}$ & [106] \\
\hline Cellulose powder & $\mathrm{Co}(\mathrm{acac})_{3}$ & $\begin{array}{l}N^{\prime} N^{\prime}-M B A \\
\text { or N-VP [99] }\end{array}$ & Grafting from & $\begin{array}{l}\text { Cellulose washed with } \mathrm{CH}_{3} \mathrm{OH}, \mathrm{C}_{3} \mathrm{H}_{6} \mathrm{O} \text {, } \\
\text { and water; then dried. Reaction under } \\
\text { nitrogen atmosphere. } \\
\text { Different temperatures, } 30-60{ }^{\circ} \mathrm{C} \text { [98], or } \\
40-50{ }^{\circ} \mathrm{C} \text { [99]; reaction carried out in water. } \\
\text { Kinetic data from 0-150 min [86], or } \\
0-120 \text { min [99]. }\end{array}$ & $\begin{array}{l}\text { Percent grafting ( } \% \mathrm{G}) \text {, true grafting }(\% \\
\mathrm{GT}) \text {, grafting efficiency }(\% \mathrm{GE}) \text {, } \\
\text { homopolymer conversion }(\% \mathrm{CH}) \text {, cellulose } \\
\text { conversion }(\% \mathrm{CC}) \text {, and total conversion ( } \% \\
\text { CT) by gravimetric methods. }\end{array}$ & {$[98,99]$} \\
\hline $\begin{array}{l}\text { Cotton linter } \\
\text { Cellulose powder }\end{array}$ & $\begin{array}{l}\text { Ceric ammonium } \\
\text { sulfate, } 1 \% \text { sulfuric acid. }\end{array}$ & $\begin{array}{l}\text { AcN } \\
\text { EA } \\
\text { MMA }\end{array}$ & Grafting from & $\begin{array}{l}\text { Cellulose treated with sodium hydroxide } \\
5-30 \% \text { wt./vol at } 25^{\circ} \mathrm{C} \text {. } \\
\text { Grafting temperatures: } 30,40 \text { and } 60^{\circ} \mathrm{C} \text {, } \\
\text { sodium bisulfite clay as initiator. } \\
\text { Polymerization in diluted } \mathrm{HCl} \text { for } 2 \mathrm{~h} \text {. }\end{array}$ & $\begin{array}{l}\text { Percent grafting ( } \% \mathrm{G}) \text {, } \\
\text { true grafting ( } \% \mathrm{GT}) \text {, grafting efficiency }(\% \\
\text { GE), by gravimetric methods. }\end{array}$ & [107] \\
\hline
\end{tabular}


Table 3. Cont.

\begin{tabular}{|c|c|c|c|c|c|c|}
\hline Backbone & $\begin{array}{l}\text { Functionalization } \\
\text { Method }\end{array}$ & Grafted Chains & $\begin{array}{l}\text { Grafting } \\
\text { Technique }\end{array}$ & Grafting Conditions & Measured Properties and Methods & Refs. \\
\hline $\begin{array}{l}\text { Hydroxypropyl } \\
\text { cellulose (HPC) }\end{array}$ & $\begin{array}{l}\text { Steglich esterification of } \\
\text { PABTC onto HPC using } \\
\text { DCC and DMAP. }\end{array}$ & $\begin{array}{l}\text { EA } \\
\text { NIPAAM }\end{array}$ & Grafting from & $\begin{array}{l}\text { Steglich esterification using DCC and } \\
\text { DMAP in chloroform at } 40^{\circ} \mathrm{C} \text {, in presence } \\
\text { of HPC and PABTC. } 6 \text { days for } 50 \% \\
\text { conversion. Polymerization of EA and } \\
\text { NIPAAM at } 60^{\circ} \mathrm{C} \text { using AIBN; } 94 \% \\
\text { conversion with free polymer in DMAc. }\end{array}$ & $\begin{array}{l}\text { Tg by DSC at } 45,55 \text { and } 135^{\circ} \mathrm{C} \text { for } \\
\text { PNIPAAM, and Tm at } 157^{\circ} \mathrm{C} \text {. TGA from } \\
\text { ambient to } 600{ }^{\circ} \mathrm{C} \text { at } 10^{\circ} \mathrm{C} / \mathrm{min} \text {, nitrogen } \\
\text { atmosphere for HPC, PINIPAAM and the } \\
\text { grafted HPC-g-PNIPAAM. }{ }^{1} \mathrm{HNMR} \text { for } \\
\text { degree of substitution. } \\
\text { SEC for HPC Macro CTA and } \\
\text { HPC-g-PNIPAAM. }\end{array}$ & [108] \\
\hline $\begin{array}{l}\text { Cellulose } \\
\text { chloroacetate } \\
\text { (CellClAc) }\end{array}$ & $\begin{array}{l}\text { Macro initiator, } \\
\mathrm{Cu}(\mathrm{I}) \mathrm{Cl} / 2^{\prime} 2^{\prime} \mathrm{BIPI} \\
\text { catalytic system via } \\
\text { ATRP controller. }\end{array}$ & $\begin{array}{l}\text { 4NPA } \\
\text { MMA }\end{array}$ & Grafting from & $\begin{array}{l}\text { ATRP of } 4 \mathrm{NPA} \text { and MMA carried out in } \\
\mathrm{DMF} \text { at } 130^{\circ} \mathrm{C} \text { for } 24 \mathrm{~h} \text {, in the presence of } \\
\mathrm{CellClAc} \text { as macro initiator, } \\
\mathrm{Cu}(\mathrm{I}) \mathrm{Cl} / 2,2^{\prime} \mathrm{BIPI} \text { catalytic system. Grafting } \\
\text { conversion under } 15 \% \text {. }\end{array}$ & NMR, FTIR, TGA, and elemental analysis. & [109] \\
\hline $\begin{array}{l}\text { Cellulosic Grewia } \\
\text { optiva fibers }\end{array}$ & $\begin{array}{l}\text { Redox initiation using } \\
\text { FAS- } \mathrm{H}_{2} \mathrm{O}_{2} \text { for grafting of } \\
\mathrm{MA} \text {, and KPS for } \\
\text { polymerization of MA. }\end{array}$ & MA & Grafting from & $\begin{array}{l}1 \mathrm{~g} \text { of mercerized Grewia optiva fibers was } \\
\text { set in distilled aqueous solution with } \\
\mathrm{NaOH} \text { for } 24 \mathrm{~h} \text {, followed by addition of } \\
\text { redox initiator }\left(\mathrm{FAS}-\mathrm{H}_{2} \mathrm{O}_{2}\right) \text {. Solution was } \\
\text { stirred for } 10 \text { min; MA was then added. } \\
\text { Solution was polymerized using } \\
\text { microwave irradiation at different times. }\end{array}$ & FTIR, SEM, TGA, swell index. & [110] \\
\hline Cellulose acetate & $\begin{array}{l}\text { Solvents: } \mathrm{DMSO}, \mathrm{PDX} \\
\text { DMAc, } \mathrm{C}_{3} \mathrm{H}_{6} \mathrm{O} \text {. } \\
\text { Initiators for grafting } \\
\text { and polymerization: } \\
\mathrm{CAN}, \mathrm{Sn}(\mathrm{Oct})_{2} \text { and } \mathrm{BPO} \text {. }\end{array}$ & MMA & Grafting from & $\begin{array}{l}\text { Focus on solvent effect. } 1.25 \mathrm{~g} \text { of cellulose } \\
\text { acetate were dissolved in } 125 \mathrm{~mL} \text { of solvent. } \\
\text { CAN or } \mathrm{Sn}(\mathrm{Oct})_{2} \text { or } \mathrm{BPO}(0.3-0.5 \mathrm{~g}) \text { were } \\
\text { added with MMA }(1.25-2 \mathrm{~mL}) \text {. Nitrogen } \\
\text { atmosphere, } 2-6 \mathrm{~h}, 30-80^{\circ} \mathrm{C} \text {, except } \\
\text { acetone, which proceeded at } 55^{\circ} \mathrm{C} \text {. }\end{array}$ & $\begin{array}{l}\text { Grafting yield (GY), total monomer } \\
\text { conversion (TC), grafting efficiency (GE) } \\
\text { and number of grafting chains per cellulose } \\
\text { acetate molecule were obtained by } \\
\text { gravimetric methods. } \\
\text { TGA, GPC, FTIR, }{ }^{1} \text { HNMR. }\end{array}$ & [111] \\
\hline $\begin{array}{l}\text { Cellulose } \\
\text { chloroacetate } \\
\text { (CellClAc) }\end{array}$ & $\begin{array}{l}\text { Macro initiator, } \\
\mathrm{Cu}(\mathrm{I}) \mathrm{Cl} / 2^{\prime} 2^{\prime} \mathrm{BIPI} \\
\text { catalytic system via } \\
\text { ATRP controller. }\end{array}$ & $\begin{array}{l}\text { NCA } \\
\text { MMA }\end{array}$ & Grafting from & $\begin{array}{l}\text { ATRP of NCA and MMA in DMF at } 130^{\circ} \mathrm{C} \text {, } \\
\text { in the presence of CellClAc. }\end{array}$ & FTIR, TGA, and elemental analysis. & [112] \\
\hline
\end{tabular}


Table 3. Cont.

\begin{tabular}{|c|c|c|c|c|c|c|}
\hline Backbone & $\begin{array}{l}\text { Functionalization } \\
\text { Method }\end{array}$ & Grafted Chains & $\begin{array}{l}\text { Grafting } \\
\text { Technique }\end{array}$ & Grafting Conditions & Measured Properties and Methods & Refs. \\
\hline $\begin{array}{l}\text { Cellulose cotton } \\
\text { fibers }\end{array}$ & $\begin{array}{l}\mathrm{Na}_{2} \mathrm{CO}_{3} \text { and thermal } \\
\text { activation. }\end{array}$ & MTC-b-CD & Grafting to & $\begin{array}{l}\text { Grafting of MCT- } \beta \text {-CD onto cotton fabric } \\
\text { carried out in alkaline medium; } 1 \mathrm{mg} \text { of } \\
\text { cellulose fiber was impregnated with } \\
\text { solutions of } 50-150 \mathrm{~g} / \mathrm{L} \text { of } \mathrm{MCT} \beta-\mathrm{CD} \text { and } \\
20-80 \mathrm{~g} / \mathrm{L} \mathrm{Na}_{2} \mathrm{CO}_{3} \text {. Solvent was } \\
\text { eliminated at room temperature before } \\
\text { heating at } 100-160^{\circ} \mathrm{C} \text {, for } 10,15 \text { and } 20 \mathrm{~min} \text {. } \\
\text { Sample were washed to obtain neutral } \mathrm{pH} \text {. } \\
\text { Silver nitrate solutions were added in situ } \\
\text { for some samples. }\end{array}$ & $\begin{array}{l}\text { FTIR and microbiological tests [113]. } \\
\text { Gravimetric methods and analytical } \\
\text { modeling [114]. MODDE software was } \\
\text { used to study the relationship between } 3 \\
\text { significant independent variables and } \\
\text { degree of grafting. }\end{array}$ & {$[113,114]$} \\
\hline $\begin{array}{l}\text { Cotton linter } \\
\text { cellulose }\end{array}$ & $\begin{array}{l}\text { CTP using APS as } \\
\text { initiator. }\end{array}$ & MMA & $\begin{array}{l}\text { Grafting from in } \\
\text { situ polymer } \\
\text { formation } \\
\text { (embedded) }\end{array}$ & $\begin{array}{l}\text { Cellulose pre-swelling: Cellulose was } \\
\text { pre-swollen in DMAc at } 160{ }^{\circ} \mathrm{C} \text { for } 0.5 \mathrm{~h} \text {. } \\
\text { Pre-swollen cellulose was filtered. A } \\
\text { solution of } \mathrm{LiCl} \text { in DMAc }(8 \% \text {, w/w) was } \\
\text { prepared. The pre-swollen cellulose was } \\
\text { added to the DMAc/LiCl solution. The } \\
\text { mixture was stirred at } 100{ }^{\circ} \mathrm{C} \text { for } 2 \mathrm{~h} \text { and } \\
\text { purged with gaseous } \mathrm{N}_{2} . \mathrm{MMA} \\
\text { polymerization was carried out at } 70-90^{\circ} \mathrm{C} \\
\text { using APS and DMSO. }\end{array}$ & TGA-DTA, FTIR, SEM, XRD. & [115] \\
\hline $\begin{array}{l}\text { Microcrystalline } \\
\text { cellulose }\end{array}$ & $\begin{array}{l}\text { Ring-opening } \\
\text { polymerization (ROP) of } \\
\text { L-LA with DMAP in an } \\
\text { ionic liquid AmimCl. }\end{array}$ & PLLA & Grafting from & $\begin{array}{l}\text { A } 4 \%(w / w) \text { microcrystalline } \\
\text { cellulose } / \text { AmimCl solution was prepared } \\
\text { and stirred at } 60^{\circ} \mathrm{C} \text {, in } \mathrm{N}_{2} \text { environment for } \\
1 \mathrm{~h} \text {. L-LA and DMAP were then added. } \\
\text { The sample was degassed } 3 \text { times in } \\
\text { vacuum } / \mathrm{N}_{2} \text { during } 1 \mathrm{~h} \text { cycles. ROP } \\
\text { proceeds at } 90^{\circ} \mathrm{C} \text { in presence of } \mathrm{N}_{2} \\
\text { atmosphere during } 11 \mathrm{~h} \text {. }\end{array}$ & $\begin{array}{l}\text { Controlled release of vitamin C. } \\
\text { Characterization by }{ }^{1} \mathrm{HNRM} \text {, UV analysis, } \\
\text { XRD, TEM, and HPLC. }\end{array}$ & [116] \\
\hline
\end{tabular}


Table 3. Cont.

\begin{tabular}{|c|c|c|c|c|c|c|}
\hline Backbone & $\begin{array}{l}\text { Functionalization } \\
\text { Method }\end{array}$ & Grafted Chains & $\begin{array}{l}\text { Grafting } \\
\text { Technique }\end{array}$ & Grafting Conditions & Measured Properties and Methods & Refs. \\
\hline $\begin{array}{l}\text { Cellulose } \\
(\mathrm{DP}=1130)\end{array}$ & $\begin{array}{l}\text { CTP; APS and MMA } \\
\text { embedded. }\end{array}$ & MMA & Grafting from & $\begin{array}{l}\text { Cellulose is pre-swollen in DMAc during } \\
30 \text { min, at } 160^{\circ} \mathrm{C} .5 \mathrm{~g} \text { of pre-swollen } \\
\text { cellulose are mixed with } 95 \mathrm{~g} \text { of water and } \\
\text { proper amounts of APS and MMA. } \\
\text { Grafting proceeds at } 80^{\circ} \mathrm{C} \text {. Excess of } \\
\text { PMMA was washed with acetone. }\end{array}$ & $\begin{array}{l}\text { Characterization: FTIR, WARD-XRD, SEM, } \\
\text { and TGA-DTA. }\end{array}$ & [117] \\
\hline $\begin{array}{l}\text { Cellulose } \\
\text { nanocrystal } \\
(\mathrm{CNC})\end{array}$ & $\begin{array}{l}\text { Macromolecular initiator } \\
\text { obtained from the } \\
\text { reaction between } \\
\text { Br-iBuBr and CNC using } \\
\text { TEA as catalyst, via } \\
\text { SI-ATRP. }\end{array}$ & $\begin{array}{l}\text { STY. } \\
\text { Cast in MMA }\end{array}$ & Grafting from & $\begin{array}{l}\text { Macromolecular initiator: } \mathrm{Br}-\mathrm{B} \mathrm{BuBr}, \mathrm{CNC} \text {, } \\
\text { and TEA were dissolved in } \mathrm{DMF} \text {, and } \\
\text { stirred under } \mathrm{N}_{2} \text { at } 70{ }^{\circ} \mathrm{C} \text { for } 24 \mathrm{~h} \text {. CNC, } \\
\text { 2-bromoisobutyrate, styrene and } \\
\text { copper(I)bromide were dissolved in anisol; } \\
\text { PMDETA was then added. The reaction } \\
\text { proceeded at } 100{ }^{\circ} \mathrm{C} \text { for } 12 \mathrm{~h} \text { and } \mathrm{N}_{2} \\
\text { atmosphere via SI-ATRP. The remanent PS } \\
\text { homopolymer was eliminated using } \\
\text { methanol, and centrifuging. The modified } \\
\text { crystals were dosed into PMMA } \\
\text { nanocomposites by solution casting. }\end{array}$ & $\begin{array}{l}\text { Characterization: TEM, SEM, FTIR, UV-Vis., } \\
\text { XRD, }{ }^{13} \text { C-NMR, TGA, DSC, and tensile test }\end{array}$ & [118] \\
\hline $\begin{array}{l}\text { Cellulose } \\
\text { nanocrys- } \\
\text { tal(CNC) }\end{array}$ & $\begin{array}{l}\text { L-LA in situ } \\
\text { polymerization using } \\
\mathrm{MgH}_{2} \text { as redox agent. } \\
\text { PLLA-g-CNC particles } \\
\text { were casted in PLLA. }\end{array}$ & PLLA & Grafting from & $\begin{array}{l}1.50 \mathrm{~g} \text { of L-LA and } 0.05 \mathrm{~g} \text { of CNCs were } \\
\text { pre-mixed. } 0.03 \mathrm{~g} \text { of } \mathrm{MgH}_{2} \text { were then } \\
\text { added and stirred during } 30 \mathrm{~min} \text {. The } \\
\text { mixture was heated to } 110^{\circ} \mathrm{C} \text { for } 3,6,10 \text {, } \\
12,18 \text { and } 24 \mathrm{~h} \text {, under nitrogen atmosphere. } \\
\text { Casting method: } 1.0 \mathrm{~g} \text { of PLLA and } 0.1 \mathrm{~g} \text { of } \\
\mathrm{CNC} \text { or } 1.3 \mathrm{~g} \text { of PLLA-g-CNC were } \\
\text { dispersed in } 10 \mathrm{~mL} \text { of chloroform or } \\
\text { toluene solution and mixed for } 10 \mathrm{~h} \text {. The } \\
\text { mixtures/solutions were poured into } \\
\text { Teflon }{ }^{\circledR} \text { plates and let stand for } 18 \mathrm{~h} \text { at } \\
80^{\circ} \mathrm{C} \text {. The films were dried under vacuum } \\
\text { at } 60^{\circ} \mathrm{C} \text { for } 72 \mathrm{~h} \text { at room temperature. }\end{array}$ & $\begin{array}{l}\text { Characterization: FTIR, }{ }^{1} \mathrm{HNRM},{ }^{13} \mathrm{C}-\mathrm{NMR} \\
\text { XRD, XPS, TGA, DSC and DMA. }\end{array}$ & [119] \\
\hline
\end{tabular}


Table 3. Cont.

\begin{tabular}{|c|c|c|c|c|c|c|}
\hline Backbone & $\begin{array}{l}\text { Functionalization } \\
\text { Method }\end{array}$ & Grafted Chains & $\begin{array}{l}\text { Grafting } \\
\text { Technique }\end{array}$ & Grafting Conditions & Measured Properties and Methods & Refs. \\
\hline $\begin{array}{l}\text { Cellulose cotton } \\
\text { fiber pulp }\end{array}$ & $\begin{array}{l}\text { (a) Esterification of } \\
\text { maleic anhydride } \\
\text { grafted onto PHA } \\
\text { (through double bond); } \\
\text { (b) PHA-g-MA is grafted } \\
\text { onto cellulose cotton } \\
\text { fiber pulp (through the } \\
\text { anhydride group). }\end{array}$ & PHA-g-MA & Grafting to & $\begin{array}{l}\text { Cotton fibers were defibered into pulp to } \\
25^{\circ} \mathrm{SR} \text { using a PL4-2 speed governing } \\
\text { beater; } 30 \mathrm{~g} / \mathrm{m}^{2} \text { paper film was then } \\
\text { formed in a RK3-KWTjul Rapid-Koethen } \\
\text { sheet former. PHA-g-MA was dissolved in } \\
\text { refluxing dichloromethane at } 60^{\circ} \mathrm{C} \text {, and } \\
\text { the paper film was dipped into the solution } \\
\text { of PHA. The PHA/CF composite film was } \\
\text { then washed with dichloromethane and } \\
\text { dried. }\end{array}$ & $\begin{array}{l}\text { Characterization: FTIR; XRD; SEM; surface } \\
\text { roughness; surface hydrophobicity, with } \\
\text { contact angle; tensile test, and TGA. }\end{array}$ & [120] \\
\hline $\begin{array}{l}\text { Cellulosic filter } \\
\text { papers }\end{array}$ & $\begin{array}{l}\text { Radiation-induced graft } \\
\text { copolymerization } \\
\text { (RIGCP) of AcN. }\end{array}$ & Acrylonitrile & Grafting from & $\begin{array}{l}\text { The reaction took place in a glass tube } \\
\text { which contained a Whatman filter paper } \\
\text { (W1) and a } 45 \% \text { AcN solution in DMF. The } \\
\text { solution was irradiated with cobalt- } 60 \\
\text { g-rays at a dose rate of } 4 \mathrm{kGy} / \mathrm{h} \text {, in air } \\
\text { atmosphere. The grafted material was } \\
\text { washed with DMF and water, followed by } \\
\text { drying }\left(T=80^{\circ} \mathrm{C}, 2 \mathrm{~h}\right) \text {. }\end{array}$ & $\begin{array}{l}\text { Degree of grafting (G\%) was determined. } \\
\text { Characterization: FTIR, XRD, XRF, TGA } \\
\text { and SEM. }\end{array}$ & [121] \\
\hline CellClAc & $\begin{array}{l}\text { ATRP grafting of the } \\
\text { studied monomers using } \\
\mathrm{CuCl}, 2^{\prime} 2^{\prime} \text { BIPI as } \\
\text { catalyst. }\end{array}$ & $\begin{array}{l}\text { NCHA, } 4 \mathrm{VP}, \mathrm{DA}, \\
\text { DAAM. }\end{array}$ & Grafting from & $\begin{array}{l}\text { Cellulose chloroacetate (ATRP } \\
\text { macroinitiator) and any of the monomers } \\
\text { (NCHA, 4VP, DAAM or DA) were added to } \\
10 \mathrm{~mL} \text { of DMF; an inert environment was } \\
\text { created using argon. The grafting reaction } \\
\text { proceeded at } 130^{\circ} \mathrm{C} \text { for } 24 \mathrm{~h} \text {. The } \\
\text { proportion of Cell.ClAc, } \mathrm{CuCl}, 2^{\prime} 2^{\prime} \mathrm{BIPI} \text { and } \\
\text { each monomer was } 1: 1: 3: 100 \text {. The reacting } \\
\text { mass proceeded to filtering and washing } \\
\text { using acetonitrile, DMF, chloroform, a } \\
\text { mixture of water-ethanol-HCl, pure water, } \\
\text { ethanol, acetone, and diethyl ether. The } \\
\text { product was dried under vacuum. }\end{array}$ & $\begin{array}{l}\text { Characterization: FTIR, UV-Visible, TGA, } \\
\text { elemental analysis, and electrical } \\
\text { conductivity. }\end{array}$ & [122] \\
\hline
\end{tabular}


Table 3. Cont.

\begin{tabular}{|c|c|c|c|c|c|c|}
\hline Backbone & $\begin{array}{l}\text { Functionalization } \\
\text { Method }\end{array}$ & Grafted Chains & $\begin{array}{l}\text { Grafting } \\
\text { Technique }\end{array}$ & Grafting Conditions & Measured Properties and Methods & Refs. \\
\hline $\begin{array}{l}\text { Regenerated } \\
\text { cellulose fibers } \\
\text { (rayon) }\end{array}$ & $\begin{array}{l}\text { Photo-chemical grafting } \\
\text { of PETA without } \\
\text { photoinitiator. }\end{array}$ & PETA & Grafting to & $\begin{array}{l}\text { Fibers were washed with a solution of } \\
\text { PETA in isopropanol. Monomer content of } \\
1 \% \text { and } 5 \% \text { was used. The cellulosic } \\
\text { material was irradiated with a broadband } \\
\mathrm{Hg} \text { lamp (emission band of } 200 \text { and } \\
300 \mathrm{~nm} \text { ), at } 50 \mathrm{~W} / \mathrm{cm} \text {. Layer deposition } \\
\text { from homo-polymerization and subsequent } \\
\text { grafting-to process onto the fiber took } \\
\text { place. }\end{array}$ & $\begin{array}{l}\text { Fiber volume content; tensile and fatigue } \\
\text { tests; SEM. }\end{array}$ & [123] \\
\hline $\begin{array}{l}\text { Cellulose } \\
\text { nanofibrils }\end{array}$ & $\begin{array}{l}\text { Nitroxide TEMPO } \\
\text { insertion and NMP of } \\
\text { HEMA }\end{array}$ & HEMA & Grafting from & $\begin{array}{l}\text { Preparation of TEMPO-oxidized cellulose } \\
\text { nanofibrils. A suspension was prepared } \\
\text { using HEMA }(0.15 \mathrm{~g}), \mathrm{H}_{2} \mathrm{O}_{2}(0.0075 \mathrm{~g}) \text {, } \\
\mathrm{CaCl}_{2}(0.0075 \mathrm{~g}) \text { and TCNF }(0.3 \mathrm{wt} . \% \text {, } \\
500 \mathrm{~g}) \text {, followed by stirring and heating at } \\
50{ }^{\circ} \mathrm{C} \text { for } 4 \mathrm{~h} \text {. Either freezer freezing, or } \\
\text { gradient freezing in liquid nitrogen were } \\
\text { used. The nanofibril suspension was set in } \\
\text { a freezer }\left(-25^{\circ} \mathrm{C}\right) \text {. The frozen material } \\
\text { pieces were } 3 \mathrm{~cm} \text { height. Drying in a } \\
\text { lyophilizer to form aerogels then took } \\
\text { place. }\end{array}$ & $\begin{array}{l}\text { Characterization: XRD; FTIR; XPS; SEM; } \\
\text { stress-strain under compression analyses; } \\
\text { BET analysis and electrical resistivity. }\end{array}$ & [124] \\
\hline
\end{tabular}


Cellulose can be extracted from lignocellulose and used as such or modified for other applications. Table 3 provides an overview of grafting of synthetic polymers onto cellulose and natural fibers. (See the tables of Section 6 for explanation of abbreviations and symbols.)

Lignin follows cellulose in abundance on earth, providing a primary natural source of aromatic compounds [125]. Several industrial applications have been attempted for lignin [55, 125-127] but not all of them have succeeded due to different reasons [125,128-130].

Marton [8] described fifty-four different constituents that can be found in lignin based on interpretation of experimental data from biochemical degradation, oxidation, and other ways of decomposition of different types of lignin materials. The combinations and proportions among these structures lead to different properties of lignin materials. Three decades later, Lewis and Sarkanen [130] organized these fifty-four structures into a map that they called phenylpropanoid pathway. As observed in Figure 3, lignins and lignans are monolignol derived compounds. Sharma and Kumar described lignin as a complex material consisting mostly of three single unit lignol precursors, coniferyl alcohol, p-coumaryl alcohol, and sinapyl alcohol, along with other atypical monolignol constitutive units in trace amounts [55].

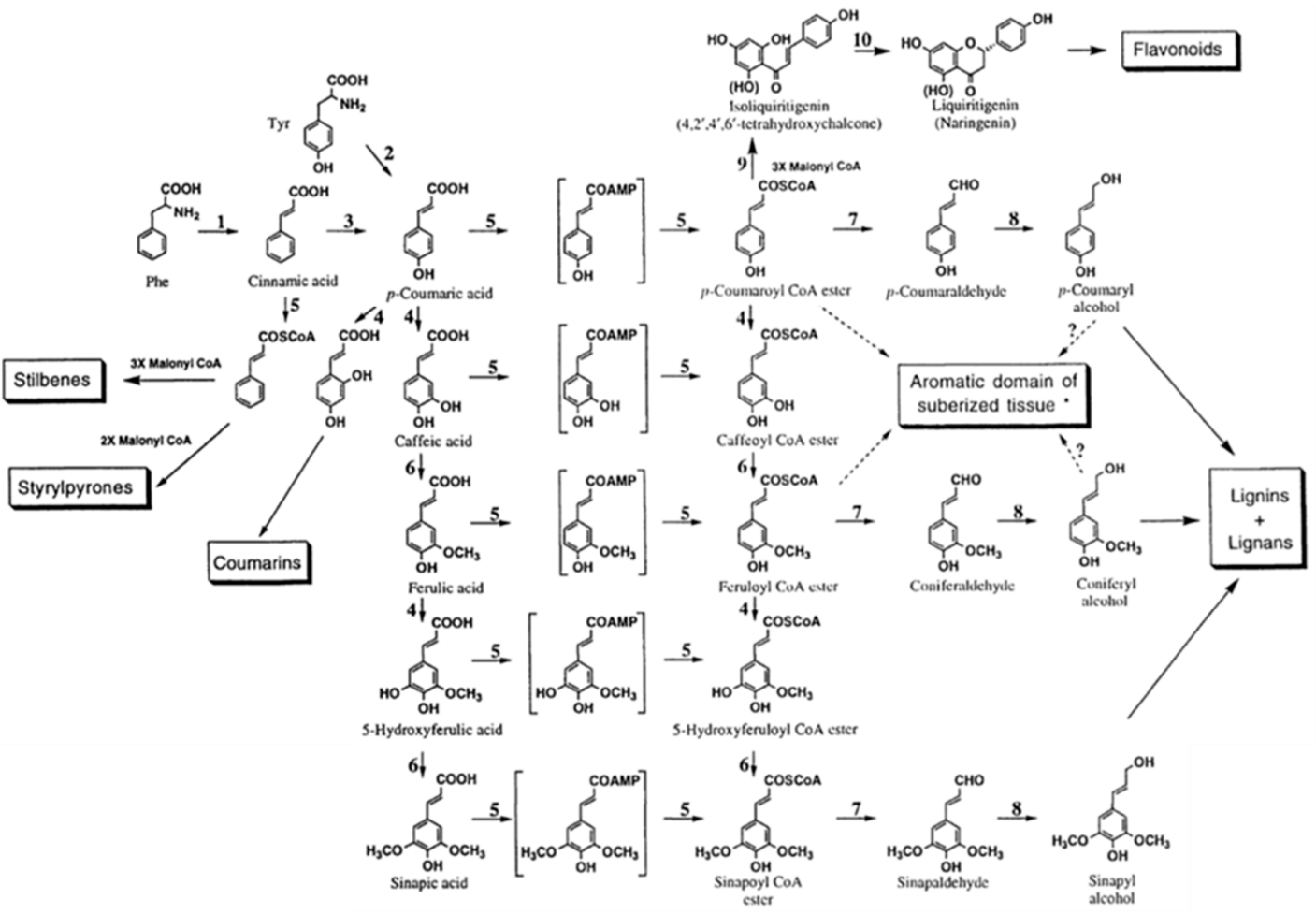

Figure 3. Main steps of the phenylpropanoid pathway: lignins and lignans are monolignol derived. 1, phenylalanine ammonia-lyase; 2, tyrosine ammonia-lyase (mostly in grasses); 3, cinnamate-4-hydroxylase; 4, hydroxylases; 5 , CoA ligases involving AMP and CoA ligation, respectively; 6, O-methyltransferases; 7, cinnamoyl-CoA:NADP oxidoreductases; 8 , cinnamyl alcohol dehydrogenases; 9 , chalcone synthase; 10 , chalcone isomerase. (Note: conversions from 7-coumaric acid to sinapic acid and corresponding CoA esters are marked in boxes since dual pathways seem to take place; *: may also involve 7-coumaryl and feruloyl tyramines, and small amounts of single unit lignols). Source: Adapted with permission from Lewis N. G. and Sarkanen S. (1998). Lignin and Lignan Biosynthesis, Washington, D.C.: Oxford University Press pp. 6-7 [130] Copyright (C) 2021 by American Chemical Society.

The process used for lignin extraction and the final properties of the material depend on the type of biomass employed [55]. Lignin is obtained from woods, which can be hard, soft, bushes, rinds, husks, corncobs, either products or residues. A pulp is obtained from these materials. The yield of lignin extraction depends on temperature, time, dispersion 
media, extraction method, and the amount of lignin present in the raw material. Lignin extraction methods can be biological or enzymatic, physical, or chemical. Integrated solutions are employed at the end to remove impurities from lignin so it can be bleached [55]. The complex structure of lignin contains specific surface moieties that provide reactive sites where polymers and other species can be synthesized, bonded, or modified [55]. These moieties were recognized as hydroxyl, carboxyl, carbonyl, and methoxyl groups.

There are two main routes for grafting of polymer chains onto lignin-based biopolymers [55]: (a) synthesis of new reactive sites within lignin's structure; and (b) modification or functionalization of lignin's hydroxyl groups. Route (a) allows lignin to become more reactive, both at the surface, and within the bulk. Polymer modification by route (a) improves both, the properties of lignin and those of the modified materials.

In route (b), a good number of functional groups can be placed in the end groups of lignin (what is sometimes referred to as the surface of lignin). Katahira et al. [131] identified seven side chain structures in the end groups of lignin: p-coumarate, ferulate, hydroxycinnamyl alcohol, hydroxycinnamaldehyde, arylglycerol, dihydrocinnamyl alcohol, and guaiacylpropane-1,3-diol end-units, as shown in Figures 4 and 5 [131]. However, it has been proposed that the phenolic hydroxyl groups shown in Figure 4, and the aliphatic hydroxyl functional groups corresponding to $\mathrm{C}-\alpha$ and $\mathrm{C}-\gamma$ positions of the side molecule fragment shown in Figure 5, are the most reactive [55]. Both routes allow one to produce grafted materials, mainly polymers, and most of them come from route (b) above $[85,132,133]$. Further reports on lignin treatments and grafting can be found elsewhere $[55,91,125,128]$.

\section{$-\mathrm{OH}$ phenolic hydroxyl groups}<smiles>COc1ccc(CCCCOc2ccc(C)cc2)cc1OC</smiles><smiles>CCOc1ccc(C(=O)CC)cc1OC</smiles><smiles>CCOc1c(OC)cc(C(=O)CC)cc1OC</smiles><smiles>COc1cc(/C=C/CO)ccc1O</smiles>

Figure 4. Repeating units in lignin. From left to right: p-hydroxyphenyl, guaiacyl, metoxy guaiacyl, syringyl, metoxi syringyl, p-coumaryl alcohol, coniferyl alcohol, synapyl alcohol. Source: Adapted with permission from Katahira et al. (2018). Lignin Valorization. Emerging Approaches: Croydon UK pp. 3 [131]. Copyright @ 2021 The Royal Society of Chemistry. 


\section{几nก lignin structure $\quad \mathrm{R}=-\mathrm{H},-\mathrm{O}-\mathrm{CH}_{3}$}

\section{$-\mathrm{OH}$ aliphatic hydroxyl groups at $\mathrm{C}-\alpha$ and $\mathrm{C}-\gamma$}
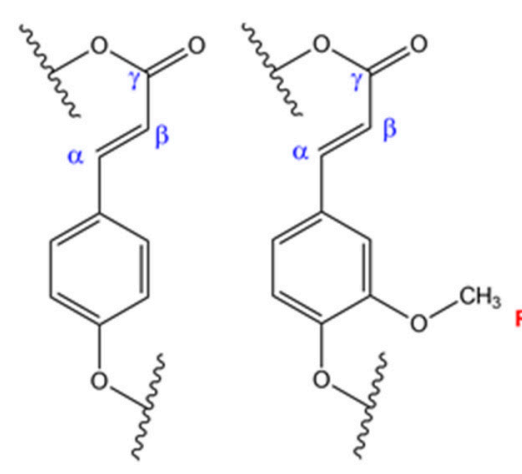<smiles>[R]c1cc(C=CCO)cc([R])c1OCC</smiles><smiles>[R]c1cc(C=CC=O)cc([R])c1OCC</smiles><smiles>[R]c1cc(CCCO)cc([R])c1OC</smiles><smiles>[R]c1cc(C(O)CCO)cc([R])c1OC</smiles><smiles>[R]c1cc(C(O)C(O)CO)cc([R])c1OC(C)C</smiles>

Figure 5. Side chain structure in end-groups in lignin. From left to right: p-coumarate, ferulate, hydroxycinnamyl alcohol, hydroxycinnamaldehyde, dihydroycinnamyl alcohol, arylpropane-1,3-diol, arylglycerol end units. Source: Adapted with permission from Katahira et al. (2018). Lignin Valorization. Emerging Approaches: Croydon UK pp. 5 [131] Copyright (C) 2021 The Royal Society of Chemistry.

The overview on grafting of synthetic polymers onto cellulose and other lignocellulosic biopolymers presented in Table 3 is further expanded in Table 4 to include other examples of lignocellulosic biomasses, and other natural biopolymers, such as polysaccharides, chitin, and chitosan. Examples of recent research reports (2020-2021) on synthesis of grafted polymers are provided in Table 5. Tables 6-13 contain extensive information related to characterization of polymer grafting. Table 14 summarizes the literature on modeling of polymer grafting. Table 15 shows the polymerization scheme of FRP including CTP and crosslinking. Finally, Tables 16-19 provide information on the many symbols and abbreviations used throughout the review. 
Table 4. Overview of grafting of synthetic polymers onto natural polymers: lignin, hemicellulose, polysaccharides, chitosan, and chitin backbones.

\begin{tabular}{|c|c|c|c|c|}
\hline Functionalization Method & Grafted Chains & Grafting Technique & Grafting Conditions & Refs. \\
\hline $\begin{array}{l}\text { Steglich esterification of RAFT } \\
\text { controller onto organosolv lignin } \\
\text { to produce a microcontroller. } \\
\text { Subsequent polymerization of } \\
\text { soybean oil derived methacrylate } \\
\text { monomers. }\end{array}$ & $\begin{array}{l}\text { Poly(soybean oil methacrylate) } \\
\text { derivatives. } \\
\text { PSBMA, PSBMAH, PSBMAEO }\end{array}$ & Grafting from & $\begin{array}{l}\text { 4-cyano-4-(phenylcarbonothioylthio) pentanoic acid was coupled with lignin via } \\
\text { Steglich esterification using DCC and DMAP at ambient conditions. } \\
\text { [Monomer]/[Lignin-RAFT]/[AIBN] molar ratio = 100:1:0.3; Temperature: } \\
70-80^{\circ} \mathrm{C} \text {; Time: } 24-48 \text { h; Conversion: } 74-94 \% \text {. }\end{array}$ & [134] \\
\hline \multirow[t]{2}{*}{$\begin{array}{l}\text { Phosphorylation. } \\
\mathrm{S}_{\mathrm{N}} 2 \text { reaction mechanism. }\end{array}$} & $\begin{array}{l}\text { Imidazole and } \mathrm{POCl}_{3} \text { react } \\
\text { with (1H-imidazol-1-yl) } \\
\text { phosphonic group, which } \\
\text { reacts with lignin. }\end{array}$ & Grafting to & $\begin{array}{l}\text { Reacted imidazole-POCl} 3 . \mathrm{DMF}, 80^{\circ} \mathrm{C}, 6 \mathrm{~h} \text {. Lignin-g- } \\
\text { (1H-imidazol-1-yl)phosphonic group. } 95^{\circ} \mathrm{C}, 12 \mathrm{~h}, \mathrm{pH}=3-4 \mathrm{in} \mathrm{DMF} \text {, yield }=92 \% \text {. } \\
\text { Composites were blended with PP-block-PE, MFI of } 1.39 \mathrm{~g} / 10 \mathrm{~min}\left(\mathrm{~T}=230^{\circ} \mathrm{C} \text {; }\right. \\
\text { standard weight of } 2.16 \mathrm{~kg}) \text {, ethylene content } 17.8 \mathrm{wt} \% \text {, from China } \\
\text { Petrochemical Co. in a Thermo Haake torque rheometer }\left(180^{\circ} \mathrm{C}: 10 \mathrm{~min} ; 60 \mathrm{rpm}\right) \text {. }\end{array}$ & {$[125,135]$} \\
\hline & Phosphorus(V) oxide & Grafting to & $\mathrm{P}_{4} \mathrm{O}_{10}$. Temperature $=20-25^{\circ} \mathrm{C}$, time $=7-8 \mathrm{~h}$. Solvent: THF. & {$[125,136,137]$} \\
\hline $\begin{array}{l}\text { Hemicellulose grafting using TBD } \\
\text { and } \varepsilon \text {-caprolactone monomer. }\end{array}$ & $\begin{array}{l}\text { PCL - Hemicellulose } \\
\text { (HC-g-PCL) }\end{array}$ & Grafting from & $\begin{array}{l}5 \mathrm{~g} \text { of dried hemicellulose }(5.00 \mathrm{~g}) \text { were introduced into a reactor containing } \\
10 \mathrm{~mL} \text { of DMSO at } 80^{\circ} \mathrm{C} \text {. Agitation at } 60 \mathrm{rpm} \text { took place until a homogeneous } \\
\text { viscous solution was obtained. Grafting temperature was } 110^{\circ} \mathrm{C} \text {. } \varepsilon \text {-caprolactone } \\
\text { monomer was added to the hemicellulose solution and agitated during } 30 \mathrm{~min} \text {. } \\
\mathrm{TBD}, 1 \% \text { of the total mass, was added. The reaction proceeded during } 4 \mathrm{hrs.} \mathrm{in} \mathrm{a} \\
\text { system with flow of nitrogen. Drying at } 60^{\circ} \mathrm{C} \text { under vacuum proceeded for } 2 \\
\text { days. FTIR, }{ }^{1} \mathrm{HRM},{ }^{13} \mathrm{CNMR}, \mathrm{HSQC}, \mathrm{GPC}, \mathrm{DSC} \text {, TGA-FTIR, Tensile Test, Contact } \\
\text { Angle, Biodegradation test. }\end{array}$ & [37] \\
\hline $\begin{array}{l}\text { Several techniques presented in } \\
\text { the review. }\end{array}$ & $\begin{array}{l}\text { Monomers or polymers used } \\
\text { in: ATRP: NIPAAM, } \\
\text { DMAEMA, STY, MMA, BA, } \\
\text { EG; RAFT: AM, AA, others; } \\
\text { ROP: PCL, L-LA, EG; Click } \\
\text { chemistry: polymer having } \\
\text { alkyne or azide groups for the } \\
\text { azide-alkyne cycloaddition, } \\
\text { EG, PCL, PLLA. }\end{array}$ & $\begin{array}{l}\text { Grafting, from; } \\
\text { grafting to; network } \\
\text { copolymer grafting }\end{array}$ & $\begin{array}{l}\text { Grafting from: ATRP, RAFT, ROP, and FRP. } \\
\text { Grafting onto: Thiol-ene reaction with photo-redox catalysis, click chemistry, } \\
\text { epoxide group-mediated reactions, condensation. } \\
\text { Network copolymer grafting: Polycondensation, free radical crosslinking. }\end{array}$ & [9] \\
\hline
\end{tabular}


Table 4. Cont

\section{Functionalization Method}

Grafting Technique

Insertion of ACX onto lignin to synthesize a RAFT

macrocontroller which

polymerized AM and AA.

RAFT polymerization of AM

and AA.

\section{Grafting Conditions}

$0.1 \mathrm{~g}$ of RAFT macroinitiator and $0.005 \mathrm{~g}$ of AIBN were added to a solution of monomer $(0.3 \mathrm{~g})$ in DMF $(4 \mathrm{~mL})$. The reactor was degassed with nitrogen for $30 \mathrm{~min}$ at ambient conditios. Reaction temperature was $70^{\circ} \mathrm{C}$, target conversion was $98 \%$ in $24 \mathrm{~h}$ for both monomers. The product was obtained by precipitation, filtration, washing and drying. Grafting was measured using ${ }^{1} \mathrm{H}$ NMR $0.2 \mathrm{~g}$ Lignin-g-Pam were dissolved in $\mathrm{KOH}$ solution ( $0.1 \mathrm{~g}$ in $1 \mathrm{~mL}$ of water), followed by heating $\left(\mathrm{T}=70{ }^{\circ} \mathrm{C}, \mathrm{t}=12 \mathrm{hrs}\right.$.), followed by neutralization with $\mathrm{HCl}$.

The solution was then precipitated into diethyl ether and dried in air. Ungrafted PAm was withdrawn by extraction with $\mathrm{CH}_{2} \mathrm{Cl}_{2}$. The product was vacuum dried, and analyzed by ${ }^{1} \mathrm{H}$ NMR and GPC, using adequate solvents.

Monomers and polymers grafted by:

FRP: Guaiacol-AM, Vainillin-LMA.

Several techniques described in the review.

Monomers copolymerized with natural extracts.

Several procedures described Grafting from Grafting through

4-propylsyringol, 4-propylguaiacol.

ADMET (Acyclic Diene Metathesis Polymerization) and ROP: Ferulic acid isorbide, butanediol.

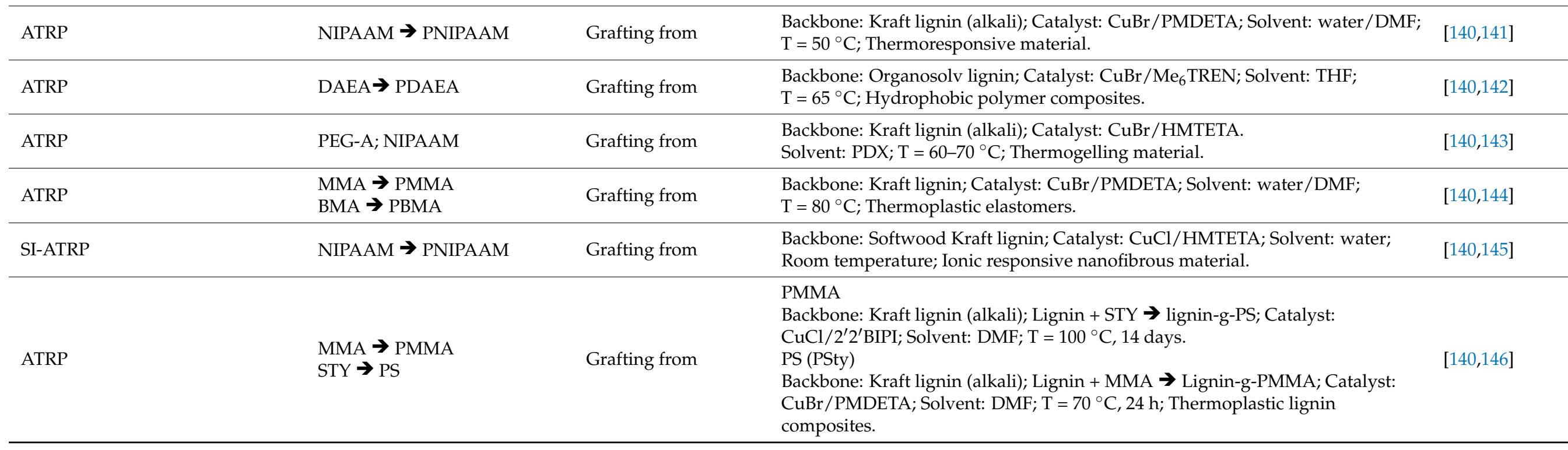


Table 4. Cont.

\begin{tabular}{|c|c|c|c|c|}
\hline Functionalization Method & Grafted Chains & Grafting Technique & Grafting Conditions & Refs. \\
\hline ATRP & $\mathrm{MMA} \rightarrow \mathrm{PMMA}$ & Grafting from & $\begin{array}{l}\text { Backbone: Kraft lignin (alkali); Lignin }+ \text { MMA } \rightarrow \text { Lignin-g-PMMA; Catalyst: } \\
\text { CuBr/PMDETA; Solvent: DMF; } \mathrm{T}=70^{\circ} \mathrm{C}, 24 \mathrm{~h} \text {; Thermoplastic lignin } \\
\text { composites. }\end{array}$ & {$[140,147]$} \\
\hline ATRP & PEGMA & Grafting from & $\begin{array}{l}\text { Backbone: Kraft lignin (alkali); Lignin-Br + PEGMA } \rightarrow \text { Lignin-g-PEGMA; } \\
\text { Catalyst: CuBr/HMTETA; Solvent: Acetone; Room temperature, overnight; } \\
\text { Supramolecular hydrogels, self-healing materials. }\end{array}$ & {$[140,148]$} \\
\hline ATRP & STY $\rightarrow$ PS & Grafting from & $\begin{array}{l}\text { Backbone: Kraft lignin (alkali); Lignin- } \mathrm{Br}+\mathrm{STY} \rightarrow \text { lignin-g-PS; Catalyst: } \mathrm{FeCl}_{3} \\
6 \mathrm{H}_{2} \mathrm{O} / \mathrm{PPh}_{3} / \text { ascorbic acid; Solvent: } \mathrm{DMF} ; \mathrm{T}=110^{\circ} \mathrm{C}, 24 \mathrm{~h} \text {; Novel } \\
\text { polymerization method. }\end{array}$ & {$[140,149]$} \\
\hline ATRP & $\mathrm{MMA} \rightarrow \mathrm{PMMA}$ & Grafting from & $\begin{array}{l}\text { Backbone: Kraft lignin (alkali); Lignin }+\mathrm{MMA} \rightarrow \text { lignin-g-PMMA; Catalyst: } \\
\mathrm{FeCl}_{3} 6 \mathrm{H}_{2} \mathrm{O} / \mathrm{PPh}_{3} / \text { ascorbic acid; Solvent: } \mathrm{DMF} ; \mathrm{T}=90^{\circ} \mathrm{C}, 24 \mathrm{~h} \text {; Novel } \\
\text { polymerization approach. }\end{array}$ & {$[140,149]$} \\
\hline ATRP & DMAEMA $\rightarrow$ PDMAEMA & Grafting from & $\begin{array}{l}\text { Backbone: Kraft lignin (alkali); Lignin-Br + DMAEMA } \rightarrow \text { lignin-g-PDMAEMA; } \\
\text { Catalyst: CuBr/HMTETA; Solvent: PDX; } \mathrm{T}=65^{\circ} \mathrm{C}, 48 \text { h; Gene delivery. }\end{array}$ & [150] \\
\hline RAFT & $\mathrm{AM} \rightarrow \mathrm{PAM}$ & Grafting from & $\begin{array}{l}\text { Backbone: Kraft lignin from softwood sources, prepared using KEX, } \\
\text { 2-Bromopropionic acid, and thionyl chloride in dry THF in } \mathrm{N}_{2} \text { atmosphere at } \\
70^{\circ} \mathrm{C} \text {, overnight. } \\
\text { Lignin-KEX + AM } \rightarrow \text { Lignin-KEX-g-PAM; RAFT controller: Lignin } \\
\text { Macrocontroller; Initiator: AIBN; Solvent: DMF; } \mathrm{T}=70^{\circ} \mathrm{C} \text {, overnight; } \\
\text { Application: Pickering emulsions. }\end{array}$ & [151] \\
\hline RAFT & $\mathrm{AM} \rightarrow \mathrm{PAM}$ & Grafting from & $\begin{array}{l}\text { Backbone: Kraft lignin. Prepared with KEX, 2-Bromopropionic acid, and thionyl } \\
\text { chloride in dry THF in } \mathrm{N}_{2} \text { atmosphere at } 70^{\circ} \mathrm{C} \text {, overnight. } \\
\text { Lignin-KEX }+\mathrm{AM} \rightarrow \text { Lignin-KEX-g-PAM; RAFT controller: Lignin } \\
\text { Macrocontroller; Initiator: AIBN. Solvent: DMF; } \mathrm{T}=70^{\circ} \mathrm{C} \text {; Application: } \\
\text { Plasticizer for Portland cement paste. }\end{array}$ & [152] \\
\hline Esterification via epoxy group & $\begin{array}{l}\text { Lignin }+ \text { GMA } \rightarrow \\
\text { Lignin-GMA + AM } \rightarrow \\
\text { Lignin-GMA-g-PAM }\end{array}$ & Grafting from & $\begin{array}{l}\text { Backbone: Kraft lignin was first functionalized by reacting with GM through the } \\
\text { epoxide ring; Lignin + GMA } \rightarrow \text { Lignin-GMA; Lignin-GMA + AM } \rightarrow \\
\text { Lignin-GMA-g-PAM; RAFT controller: Lignin Macrocontroller; Initiator: AIBN; } \\
\text { Solvent: DMF; T }=70^{\circ} \mathrm{C} \text {; Application: Plasticizer for Portland cement paste. }\end{array}$ & [153] \\
\hline
\end{tabular}


Table 4. Cont.

\begin{tabular}{|c|c|c|c|c|}
\hline Functionalization Method & Grafted Chains & Grafting Technique & Grafting Conditions & Refs. \\
\hline RAFT & $\begin{array}{l}\text { Lignin }+ \text { ACX } \rightarrow \text { Lignin-ACX } \\
+ \text { AM } \rightarrow \text { Lignin-ACX-g-PAM }\end{array}$ & Grafting from & $\begin{array}{l}\text { Backbone: Kraft lignin; Lignin }+ \text { ACX } \rightarrow \text { Lignin-ACX; Lignin-ACX }+ \text { AM } \rightarrow \\
\text { Lignin-ACX-g-PAM; RAFT controller: Lignin Macrocontroller; Initiator: AIBN; } \\
\text { Solvent: DMF; } \mathrm{T}=70^{\circ} \mathrm{C} \text {; Application: Cationic flocculant. }\end{array}$ & [153] \\
\hline RAFT & $\begin{array}{l}\text { Lignin }+ \text { XCA } \rightarrow \text { Lignin-XCA } \\
+ \text { DMC } \rightarrow \\
\text { Lignin-XCA-g-PDMC }\end{array}$ & Grafting from & $\begin{array}{l}\text { Backbone: Kraft lignin; Lignin }+ \text { XCA } \rightarrow \text { Lignin-XCA; Lignin-XCA + DMC } \rightarrow \\
\text { Lignin-XCA-g-PDMC; RAFT controller: Lignin; Macrocontroller prepared by } \\
\text { Steglich esterification using DCC and DMAP for } 48 \text { h; Initiator: AMBN. Solvent: } \\
\text { DMF; } T=70^{\circ} \mathrm{C} \text {, under nitrogen atmosphere; Application: Cationic flocculant. }\end{array}$ & [154] \\
\hline ROP & $\begin{array}{l}\text { Sulfonated Lignin }+ \text { MOX } \rightarrow \\
\text { Lignin-g-PMOX }\end{array}$ & Grafting from & $\begin{array}{l}\text { Backbone: Lignophenol from Japanese cedar; Initiator: Tosylated lignin; } \\
\text { Monomer: MOX; Solvent: DMSO; } \mathrm{T}=100^{\circ} \mathrm{C}, 10 \mathrm{~h} \text {; Application: Anti-ineffective } \\
\text { ointment. }\end{array}$ & [156] \\
\hline ROP & $\begin{array}{l}\text { Indulin AT lignin }+ \text { L-LA } \rightarrow \\
\text { Indulin AT lignin-g-PLLA }\end{array}$ & Grafting from & $\begin{array}{l}\text { Backbone: Indulin AT lignin; Initiator: Triazabicyclodecene; Monomer: L-LA; } \\
\text { Reaction carried out in bulk; } \mathrm{T}=135^{\circ} \mathrm{C} \text {; Application: composites. }\end{array}$ & [157] \\
\hline ROP & $\begin{array}{l}\text { Biobutanol lignin }+\mathrm{CL} \rightarrow \\
\text { Biobutanol lignin }-\mathrm{g}-\mathrm{PCL}\end{array}$ & Grafting from & $\begin{array}{l}\text { Backbone: Biobutanol lignin; Initiator: Triazabicyclodecene; Monomer: CL; } \\
\text { Reaction carried out in bulk; } \mathrm{T}=135^{\circ} \mathrm{C} \text {; Application: composites. }\end{array}$ & [158] \\
\hline ROP & $\begin{array}{l}\text { Lignin (organosolv) }+\mathrm{CL}+ \\
\text { L-LA } \rightarrow \text { Lignin-g-PCL/PLLA }\end{array}$ & Grafting from & $\begin{array}{l}\text { Backbone: Lignin orgaosolv; Initiator: } \mathrm{Sn}(\mathrm{Oct})_{2}(\operatorname{tin}(\mathrm{II}) 2 \text {-ethylhexanoate) and } \\
\text { alkyl alcohol functional groups on lignin; Monomer: } \mathrm{CL} \text { and L-LA (ratio 2:1); } \\
\text { Solvent: Toluene; } \mathrm{T}=120^{\circ} \mathrm{C} \text {, under Ar atmosphere, during } 45 \text { h; Application: } \\
\text { composites. }\end{array}$ & [159] \\
\hline ROP & $\begin{array}{l}\text { Alkali Lignin }+ \text { B-BL } \rightarrow \\
\text { Lignin-g-PHB }\end{array}$ & Grafting from & $\begin{array}{l}\text { Backbone: Lignin alkali; Initiator: } \mathrm{Sn}(\mathrm{Oct})_{2}(\operatorname{tin}(\mathrm{II}) 2 \text {-ethylhexanoate) and alkyl } \\
\text { alcohol functional groups on lignin; Monomer: B-BL; Reaction carried out in } \\
\text { bulk; } \mathrm{T}=130^{\circ} \mathrm{C} \text {, under nitrogen atmosphere, during } 24 \mathrm{~h} \text {; Application: } \\
\text { Nano-fibers for medical applications. }\end{array}$ & [160] \\
\hline ROP & $\begin{array}{l}\text { Lignin alkali }+ \text { CL } \rightarrow \\
\text { Lignin-g-PCL }\end{array}$ & Grafting from & $\begin{array}{l}\text { Backbone: Lignin Alkali; Initiator: } \mathrm{Sn}(\mathrm{Oct})_{2}(\operatorname{tin}(\mathrm{II}) 2 \text {-ethylhexanoate) and alkyl } \\
\text { alcohol functional groups on lignin; Monomer: } \mathrm{CL} \text {; Reaction carried out in bulk; } \\
\mathrm{T}=50^{\circ} \mathrm{C} \text {, under nitrogen atmosphere, during } 14-62 \mathrm{~h} \text {; Application: Nano-fibers } \\
\text { for medical applications. }\end{array}$ & [161] \\
\hline
\end{tabular}


Table 4. Cont

\begin{tabular}{|c|c|c|c|c|}
\hline Functionalization Method & Grafted Chains & Grafting Technique & Grafting Conditions & Refs. \\
\hline ROP & $\begin{array}{l}\text { Lignin alkali }+\mathrm{CL} \rightarrow \\
\text { Lignin-g-PCL }\end{array}$ & Grafting from & $\begin{array}{l}\text { Backbone: Lignin alkali; Initiator: } \mathrm{Sn}(\mathrm{Oct})_{2}(\mathrm{tin}(\mathrm{II}) 2 \text {-ethylhexanoate) and alkyl } \\
\text { alcohol functional groups on lignin; Monomer: } \mathrm{CL} \text {; Reaction carried out in bulk; } \\
\mathrm{T}=50^{\circ} \mathrm{C} \text {, under nitrogen atmosphere, during } 14-62 \mathrm{~h} \text {; Application: Nano-fibers } \\
\text { for medical applications. }\end{array}$ & [162] \\
\hline ROP & $\begin{array}{l}\text { Softwood Kraft lignin from } \\
\text { UPM BioPiva }+ \text { EOX } \rightarrow \\
\text { Lignin-g-PEOX }\end{array}$ & Grafting from & $\begin{array}{l}\text { Backbone: Softwood Kraft lignin from UPM BioPiva; Initiator: P4-t-Bu; } \\
\text { Monomer: CL; Solvent: Toluene; } \mathrm{T}=180^{\circ} \mathrm{C} \text {, under Ar atmosphere, during } 16 \mathrm{~h} \text {; } \\
\text { Application: Non-ionic surfactants. }\end{array}$ & [163] \\
\hline $\begin{array}{l}\text { Embedded polymerization and } \\
\text { further crosslinking. }\end{array}$ & $\begin{array}{l}\text { Chitosan; N-VP; } 4 \mathrm{VP} ; \\
\text { Crosslinking agent: } \\
\text { N'N'-MBA }\end{array}$ & Grafting from & $\begin{array}{l}1.5 \mathrm{~g} \text { of chitosan were dried at } 40-50^{\circ} \mathrm{C} \text { during } 24 \mathrm{~h} \text { and suspended in } 150 \mathrm{~mL} \text { of } \\
2 \% \mathrm{CH}_{3} \mathrm{COOH} \text { for } 1 \text { to } 2 \mathrm{~h} .2 \mathrm{~mL} \text { of monomers in a } 1: 1 \text { ratio were incorporated } \\
\text { and stirred for } 30 \mathrm{~min} \text {. } 0.15 \mathrm{~mol} / \mathrm{L} \text { AIBN were then added and stirred for } 2 \text { more } \\
\text { hours. } \mathrm{T}=65^{\circ} \mathrm{C} \text {, under air environment. Product washed using several solvents, } \\
\text { and dried at } 500^{\circ} \mathrm{C} \text {. The yield was } 78-86 \% \text {. A solution of grafted chitosan in } \\
\text { acetic acid }(0.1 \% \mathrm{v} / \mathrm{v}) \text { was heated at } 70^{\circ} \mathrm{C} ; \mathrm{N}^{\prime} \mathrm{N}^{\prime} \text {-MBA was then added and } \\
\text { allowed to react during } 30 \mathrm{~min} \text {. }\end{array}$ & [164] \\
\hline $\begin{array}{l}\text { RAFT; Steglich esterification of } \\
\text { phthalic anhydride and } \\
\text { 4,4-Azobis(4-cyanovaleric acid). }\end{array}$ & $\begin{array}{l}\text { Chitosan }+ \text { Phthalic } \\
\text { Anhydride } \rightarrow \text { (DCC/DMAP) } \\
\rightarrow \text { Chitosan-Phthaloylated } \\
\text { Chitosan-Phthaloylated }+ \\
\text { 4,4-Azobis(4-cyanovaleric } \\
\text { acid } \rightarrow \text { (DCC/DMAP) } \rightarrow \\
\text { Chitosan-Phthaloylate-RAFT. } \\
\text { Chitosan-Phthaloylate-RAFT + } \\
\text { NIPAAM } \rightarrow \\
\text { Chitosan-Phthaloaylte-RAFT- } \\
\text { g-NIPAAM. }\end{array}$ & Grafting from & $\begin{array}{l}\text { Phthaloylation of chitosan: A solution of phthalic anhydride }(5.5 \mathrm{mmol}) \text { in } 6 \mathrm{~mL} \\
\text { of DMF/H2O }(95 / 5 \mathrm{v} / \mathrm{v} \%) \text { was prepared, followed by the addition of chitosan } \\
\text { powder in } 1.86 \mathrm{mmol} \text {. The system was purged by } \mathrm{N}_{2} \text { for } 0.5 \mathrm{~h} \text {. Heating at } 120^{\circ} \mathrm{C} \\
\text { was maintained during } 8 \mathrm{~h} \text {. } \\
\text { The phthaloylated chitosan }(1 \mathrm{mmol}) \text { was mixed with } 1.2 \mathrm{mmol} \text { and } \\
\text { 4,4-azobis( } 4 \text {-cyanovaleric acid).in } 30 \mathrm{~mL} \text { of dried DMF in the presence of } \\
\text { carbonyl activating reagents of DCC }(1 \mathrm{mmol}) \text { and } 4 \text {-DMAP }(0.12 \mathrm{mmol}) \text { as } \\
\text { catalysts. Reaction at room temperature for } 10 \text { days. } \\
\text { Chitosan-Phthaloylate-RAFT ( } 0.0468 \mathrm{~g}) \text { and dry DMF }(5 \mathrm{~mL}) \text { were mixed by } \\
\text { stirring under nitrogen atmosphere. After complete dissolution, initiator } \\
4,4 \text {-Azobis }(4 \text {-cyanovaleric acid) }(0.01 \text { mmol) and NIPAAM monomer }(0.50 \mathrm{~g} \text {; } \\
4.4 \text { mmol) were incorporated. Polymerization conducted at } 70{ }^{\circ} \mathrm{C} \text { for } 48 \mathrm{~h} .\end{array}$ & [165] \\
\hline Cerium (IV) attack & Chitin + acrylic monomers. & Grafting onto & $\begin{array}{l}\text { Cerium (IV); Chitin + AM } \rightarrow \text { Chitin-g-PAM; Chitin + AA } \rightarrow \text { Chitin-g-PAA; } \\
\text { Chitin + MA } \rightarrow \text { Chitin-g-PMA; Chitin + MMA } \rightarrow \text { Chitin-g-PMMA }\end{array}$ & [58] \\
\hline$\gamma$-Radiation & Chitin + STY (radiated) & Grafting onto & Chitin + STY $\rightarrow$ Chitin-g-PS & [58] \\
\hline
\end{tabular}


Table 4. Cont.

\section{Functionalization Method}

ROP

Cyclic and vinyl monomers

Modified Chitin

Chitosan $+\mathrm{AA}+\mathrm{TiO}_{2}$

Chitosan-g-PAA-TIO

Chitosan $+\mathrm{CL} \rightarrow$

Chitoan-g-PCL

\section{Grafting Technique}

\section{Grafting Condition}

Surface-initiated ROP graft copolymerization of L-LA and CL with initiation by hydroxy groups on chitin nanofiber, catalyzed by tin(II) 2-ethylhexanoate was performed, obtaining chitin nanofiber-graft-PLA-co-PL, which is a known

biodegradable polyester. Chitin was soaked in a solution of LA/CL in toluene tin(II) 2-ethylhexanoate (ca. $5 \mathrm{~mol} \%$ ) was incorporated. Heating at $80{ }^{\circ} \mathrm{C}$ was maintained during $48 \mathrm{~h}$.

A solution of CS-PAAc (1:1 mass/vol) was prepared with different contents of $\mathrm{TiO}_{2} ; 0.0,1.0,2.0$ and $3.0 \mathrm{wt} . \%$ of the total polymer concentration was

incorporated. Each solution was sonicated in a bath for $15 \mathrm{~min}$. The solutions were transferred into small glass vials and were subjected to 60 Co-gamma rays at irradiation dose of $30 \mathrm{kGy}$.

$0.5 \mathrm{~g}$ chitosan and $15 \mathrm{~mL} \mathrm{MeSO}{ }_{3} \mathrm{H}$ were placed in a round bottom flask unde nitrogen atmosphere at $45^{\circ} \mathrm{C}$ for $0.5 \mathrm{~h} .4 .19 \mathrm{~g} \mathrm{CL}$ monomers were fed to the reactor, followed by stirring and reaction during $4.5 \mathrm{~h}$.

\section{Refs.}

Grafting from

Grafting from

Grafting from


Polysaccharides are abundant in nature, no matter whether in plants or animals, and are important for in vivo functions. As observed in Figure 6, there is a wide variety of polysaccharides, but the most abundant ones are cellulose and chitin. Cellulose provides structure to the cell walls of plants. Chitin, on the other side, is part of the exoskeletons of crustaceans, shellfish, and insects [169].

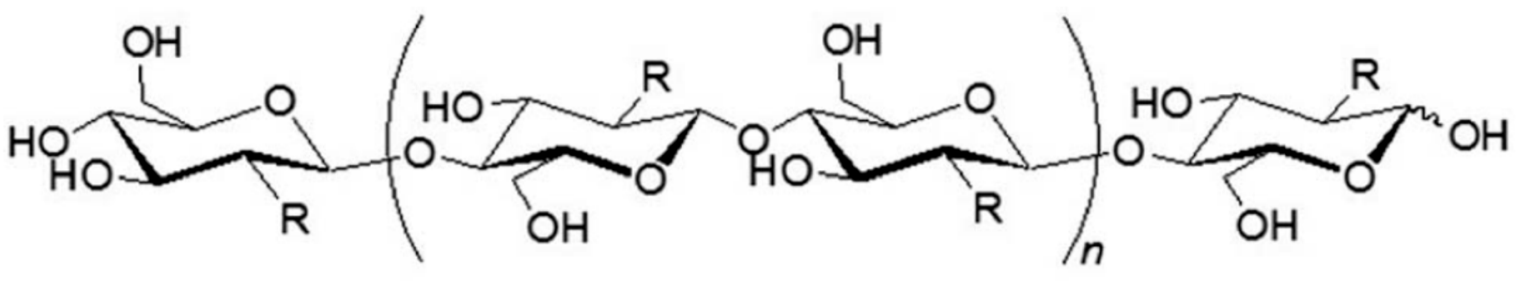

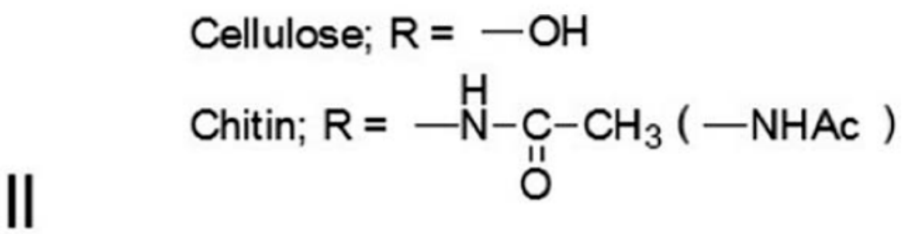

\section{Reducing terminus}
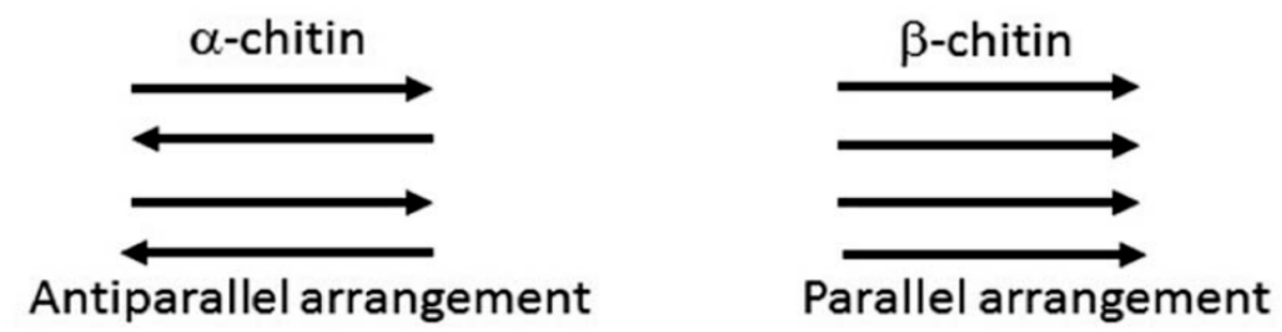

Figure 6. Cellulose and chitin structures (top). II: schematic diagram of crystalline structures for different forms of chitin. Source: Adapted with permission from Jun-ichi Kadokawa (2015). Fabrication of nanostructured and microstructured chitin materials through gelation with suitable dispersion media: RSC Adv. 512736 [169]. Copyright @ 2021 The Royal Society of Chemistry.

Chitin is an abundant but only marginally used biomass. There are several reasons why not many practical applications for chitin have been developed [169]: (a) bulky structure; (b) insolubility in water and typical organic solvents; (c) it is harmful to recover chitin, since the available procedures require the use of strong acids and bases; (d) native chitin from crustaceans, which have exoskeletons that protect animals from their predators, has fibrous structures rich in proteins and minerals; and (e) there is a need to remove mineral and protein constituents in order to isolate chitin.

Chitosan was developed to overcome the drawbacks of chitin. Chitosan is commercially attractive for production of biocompatible polymers for environmental and biomedical applications [30]. It is basically a copolymer of N-acetyl-D-glucosamine and D-glucosamine. It is obtained from the hydration of chitin. This hydration takes place in alkaline solutions in a temperature range of $80-140{ }^{\circ} \mathrm{C}$ during $10 \mathrm{~h}[170,171]$.

Chitosan is a cationic polysaccharide, produced by deacetylation of chitin. Deacetylation proceeds to different levels depending on the intended uses. The physical properties of chitosan, particularly solubility, depend on molecular weight and degree of deacetylation of the material [172-174].

Modification of chitosan leads to a diversity of derivates, with differentiated properties. As shown in Figure 1 of Deng et al. [174], different chitosan moieties are possible. Each one of them is produced from grafting or other chemical or enzymatic modification forms. 
They differ in antimicrobial activity. Further studies on chitin-chitosan modification are available in the literature $[58,175-178]$.

\subsection{Polymer Backbones}

Polymer backbones are the most common substrates for grafting modification. Several techniques can be used to create many possible combinations. Most of these developments are focused on property improvement for industrial applications. They include synthesis of adhesives; reinforcement of mechanical properties; improvement of chemical resistance; synthesis of electro, optical, thermo responsive polymers; health care applications; and self-healing polymers, among others [179].

Polymer grafting techniques have been reported since the late 1950s $[8,9,11]$. Polymers based on acrylamide and acrylonitrile using polymer grafting techniques are included in the early reports. However, the huge increase in research related to synthesis of materials with controlled microstructures using polymer grafting techniques has been possible due to the advances in reversible deactivation radical polymerization (RDRP) techniques over the last three decades [180-184]. The main RDRP routes are nitroxide mediated polymerization (NMP) [185], reversible addition fragmentation transfer polymerization (RAFT) $[180,186,187]$, and atom transfer radical polymerization (ATRP) [71,188], although there are other polymer synthesis techniques, such as ring-opening polymerization (ROP) [181] and click chemistry, among others, that can be used. Another important aspect in polymer grafting is the solvent used for the reaction, particularly when grafting proceeds as a heterogenous process. As observed in Table 19, solvents such as supercritical fluids, mainly supercritical carbon dioxide, water, DMF, or combinations of solvents are typically used for polymer grafting. These techniques have improved our skills to produce molecularly well-defined, chain-end tethered polymer brush films. The assets of RDRP have substantially impacted the synthesis and properties of surface-grafted polymers. Although vinyl monomers are widely use in graft polymerization for backbones or side-arms, other monomers coming from natural sources are increasingly being used. That is the case, for instance, of $\varepsilon$-caprolactone, lactic acid [189], L-lactide, and butyrolactone. It is also observed in Table 19 that other nontraditional monomers such as acrylamide (AM), $\mathrm{N}$-isopropylacrylamide (NIPAAM), and acrylates and methyl acrylates, such as methyl methacrylate (MMA) and acrylic acid (AA), are being increasingly used in polymer grafting applications. To get a glimpse of the focus of research papers that involve polymer grafting as the chemical route for polymer modification, Table 5 summarizes journal reports on polymer grafting from the current period (2020-2021). As expected, an increasing trend toward the improvement of natural biopolymers using synthetic polymer arms is observable.

Table 5. Recent reports on the production of materials using polymer grafting (2020-2021).

\begin{tabular}{ll}
\hline Title ${ }^{\text {a }}$ & Reference \\
\hline $\begin{array}{l}\text { Synthesis of diallyl dimethyl ammonium chloride grafted polyvinyl pyrrolidone (PVP-g-DADMAC) and its } \\
\text { applications. }\end{array}$ & [190] \\
\hline $\begin{array}{l}\text { Synthesis and characterization of biocompatible hydrogel based on hydroxyethyl cellulose-g-poly(hydroxyethyl } \\
\text { methacrylate). }\end{array}$ & [191] \\
\hline $\begin{array}{l}\text { Poly(vinylidene fluoride) (PVDF)/PVDF-g-polyvinylpyrrolidone (PVP)/TiO2 mixed matrix nanofiltration } \\
\text { membranes: preparation and characterization. }\end{array}$ & [192] \\
\hline $\begin{array}{l}\text { Synthesis and characterization of poly (acrylonitrile-g-lignin) by semi-batch solution polymerization and evaluation } \\
\text { of their potential application as carbon materials }\end{array}$ & [193] \\
\hline $\begin{array}{l}\text { High crosslinked sodium carboxyl methylstarch-g-poly (acrylic acid-co-acrylamide) resin for heavy metal } \\
\text { adsorption: its characteristics and mechanisms. }\end{array}$ & [194] \\
\hline $\begin{array}{l}\text { Preparation and characterization of PLLA/chitosan-graft-poly ( } \varepsilon \text {-caprolactone) (CS-g-PCL) composite fibrous mats: } \\
\text { The microstructure, performance and proliferation assessment. }\end{array}$ & [168] \\
\hline $\begin{array}{l}\text { Synthesis of partly debranched starch-g-poly(2-acryloyloxyethyl trimethyl ammonium chloride) catalyzed by } \\
\text { horseradish peroxidase and the effect on adhesion to polyester/cotton yarn. }\end{array}$ & [195] \\
\hline
\end{tabular}


Table 5. Cont.

Title $^{\text {a }}$

Reference

Cellulose Acetate thermoplastics with high modulus, dimensional stability and anti-migration properties by using

CA-g-PLA as macromolecular plasticizer.

Superwettable PVDF/PVDF- g-PEGMA ultrafiltration membranes.

[196]

[197]

Removal of malachite green using carboxymethyl cellulose-g-polyacrylamide/montmorillonite nanocomposite hydrogel.

Ultraviolet illumination responsivity of the $\mathrm{Au} / \mathrm{n}$-Si diodes with and without poly (linolenic acid)-g-poly (caprolactone)-g-poly (t-butyl acrylate) interfacial layer.

Synthesis of CO2-based polycarbonate-: G -polystyrene copolymers via NMRP.

Compatibilization of iPP/HDPE blends with PE-g-iPP graft copolymers.

Preparation, characterization of feather protein-g-poly(sodium allyl sulfonate) and its application as a low-temperature adhesive to cotton and viscose fibers for warp sizing.

Semi-Natural superabsorbents based on starch-g-poly(acrylic acid): Modification, synthesis and application.

Design and development of polymethylmethacrylate-grafted gellan gum (PMMA-g-GG)-based pH-sensitive novel drug delivery system for antidiabetic therapy.

Preparation and characterization of bioinert amphiphilic P(VDF-co-CTFE)-g-POEM graft copolymer.

Preparation, characterization of poly(acrylic acid)-g-feather protein-g-poly(methyl acrylate) and application in improving adhesion of protein to PLA fibers for sizing.

Antibacterial and pH-responsive quaternized hydroxypropyl cellulose-g-poly(THF-co-epichlorohydrin) graft copolymer: Synthesis, characterization and properties.

Thermally self-assembled biodegradable poly(casein-g-N-isopropylacrylamide) unimers and their application in drug delivery for cancer therapy.

Swelling capacity of sugarcane bagasse-g-poly(acrylamide)/attapulgite superabsorbent composites and their application as slow release fertilizer

Structure formation in $\mathrm{pH}$-sensitive micro porous membrane from well-defined ethyl cellulose-g-PDEAEMA via non-solvent-induced phase separation process

Micelles with a loose core self-assembled from coil-g-rod graft copolymers displaying high drug loading capacity.

Synthesis and water absorbing properties of KGM-g-P(AA-AM-(DMAEA-EB)) via grafting polymerization method.

Preparation of hydrophilic woven fabrics: Surface modification of poly(ethylene terephthalate) by grafting of poly(vinyl alcohol) and poly(vinyl alcohol)-g-(N-vinyl-2-pyrrolidone).

The preparation, physicochemical and thermal properties of the high moisture, solvent and chemical resistant starch-g-poly(geranyl methacrylate) copolymers.

Reverse poly( $\varepsilon$-caprolactone)-g-dextran graft copolymers. Nano-carriers for intracellular uptake of anticancer drugs.

High-performance solid-state bendable supercapacitors based on PEGBEM-g-PAEMA graft copolymer electrolyte.

Design of well-defined polyethylene-g-poly-methyltrifluorosiloxane graft copolymers via direct copolymerization of ethylene with polyfluorosiloxane macromonomers.

Synthesis and characterization of poly(vinyl chloride-g- $\varepsilon$-caprolactone) brush type graft copolymers by ring-opening polymerization and "click" chemistry.

Adhesion of cornstarch-g-poly (2-hydroxyethyl acrylate) to cotton fibers in sizing.

Polynorbornene-g-poly(ethylene oxide) through the combination of ROMP and nitroxide radical coupling reactions. [220]

Potent bioactive bone cements impregnated with polystyrene-g-soybean oil-AgNPs for advanced bone tissue applications.

[220]

Fabrication of cellulose nanocrystal-g-poly(acrylic acid-co-acrylamide) aerogels for efficient $\mathrm{Pb}(\mathrm{II})$ removal.

The compatibilization of PLA-g-TPU graft copolymer on polylactide/thermoplastic polyurethane blends.

Cellulose-g-poly-(acrylamide-co-acrylic acid) polymeric bioadsorbent for the removal of toxic inorganic pollutants from wastewaters

Performance improvement for thin-film composite nanofiltration membranes prepared on PSf/PSf-g-PEG blended substrates. 
Table 5. Cont.

\begin{tabular}{|c|c|}
\hline Title $^{a}$ & Reference \\
\hline $\begin{array}{l}\text { Synthesis and characterization of poly(vinyl chloride-g-methyl methacrylate) graft copolymer by redox } \\
\text { polymerization and Cu catalyzed azide-alkyne cycloaddition reaction. }\end{array}$ & [226] \\
\hline $\begin{array}{l}\text { Encapsulation of NPK fertilizer for slow release using sodium carboxymethyl cellulose-g-poly } \\
\text { (AA-C0-AM-C0-AMPS)/ Montmorillonite clay-based nanocomposite hydrogels for sustainable agricultural } \\
\text { applications. }\end{array}$ & [227] \\
\hline $\begin{array}{l}\text { Engineering a "PEG-g-PEI/DNA nanoparticle-in- PLGA microsphere" hybrid-controlled release system to enhance } \\
\text { immunogenicity of DNA vaccine. }\end{array}$ & [228] \\
\hline $\begin{array}{l}\text { Chitosan-g-oligo(L,L-lactide) copolymer hydrogel for nervous tissue regeneration in glutamate excitotoxicity: } \\
\text { In vitro feasibility evaluation }\end{array}$ & [229] \\
\hline
\end{tabular}

Table 6. Common characterization methods.

\begin{tabular}{ll}
\hline Method & Property Measured \\
\hline $\begin{array}{l}\text { Nuclear magnetic resonance } \\
\text { (NMR) }\end{array}$ & $\begin{array}{l}\text { NMR identifies the nature of the group and the site where the graft is attached to the polymer } \\
\text { backbone. }{ }^{1} \mathrm{H} \text { NMR and }{ }^{13} \mathrm{C} \text { NMR are utilized depending on the chemical nature of the material } \\
{[73] .}\end{array}$ \\
\hline $\begin{array}{l}\text { Infrared spectroscopy (IR) } \\
\text { grafting functionalities in modified polyolefins by determining the intensity of the characteristic } \\
\text { reference bands in the sample, and compare them to a calibration curve of known concentrations } \\
\text { of the same functional group [73]. }\end{array}$ \\
$\begin{array}{ll}\text { Scanning electron } \\
\text { microscopy (SEM) }\end{array}$ & $\begin{array}{l}\text { SEM is useful for the study of surface morphologies of grafted materials; also used as a tool to } \\
\text { confirm that grafting has taken place [59]. }\end{array}$ \\
\hline X-ray diffraction (XRD) & $\begin{array}{l}\text { XRD is useful for the study of crystallinity of chemical substances. Polymer grafting is associated } \\
\text { with changes in XRD patterns [59]. }\end{array}$ \\
\hline $\begin{array}{l}\text { Size exclusion } \\
\text { chromatography (SEC) }\end{array}$ & $\begin{array}{l}\text { It allows determination of molecular weight averages (MW) and molecular weight distributions } \\
\text { (MWD) of grafted chains [230], which must be separated from the backbone before carrying out } \\
\text { the analyses to focus on the grafts only. }\end{array}$ \\
\hline $\begin{array}{l}\text { Differential scanning } \\
\text { calorimetry (DSC) }\end{array}$ & $\begin{array}{l}\text { This is utilized to evaluate changes in crystallinity through the thermal behavior between } \\
\text { backbones and grafted materials. }\end{array}$ \\
\hline $\begin{array}{l}\text { Thermogravimetric analysis } \\
\text { (TGA) }\end{array}$ & $\begin{array}{l}\text { This is used to study changes in thermal decomposition profiles between backbones and grafted } \\
\text { materials. }\end{array}$ \\
\hline
\end{tabular}


Table 7. Summary of thermal characterization techniques employed in grafted materials.

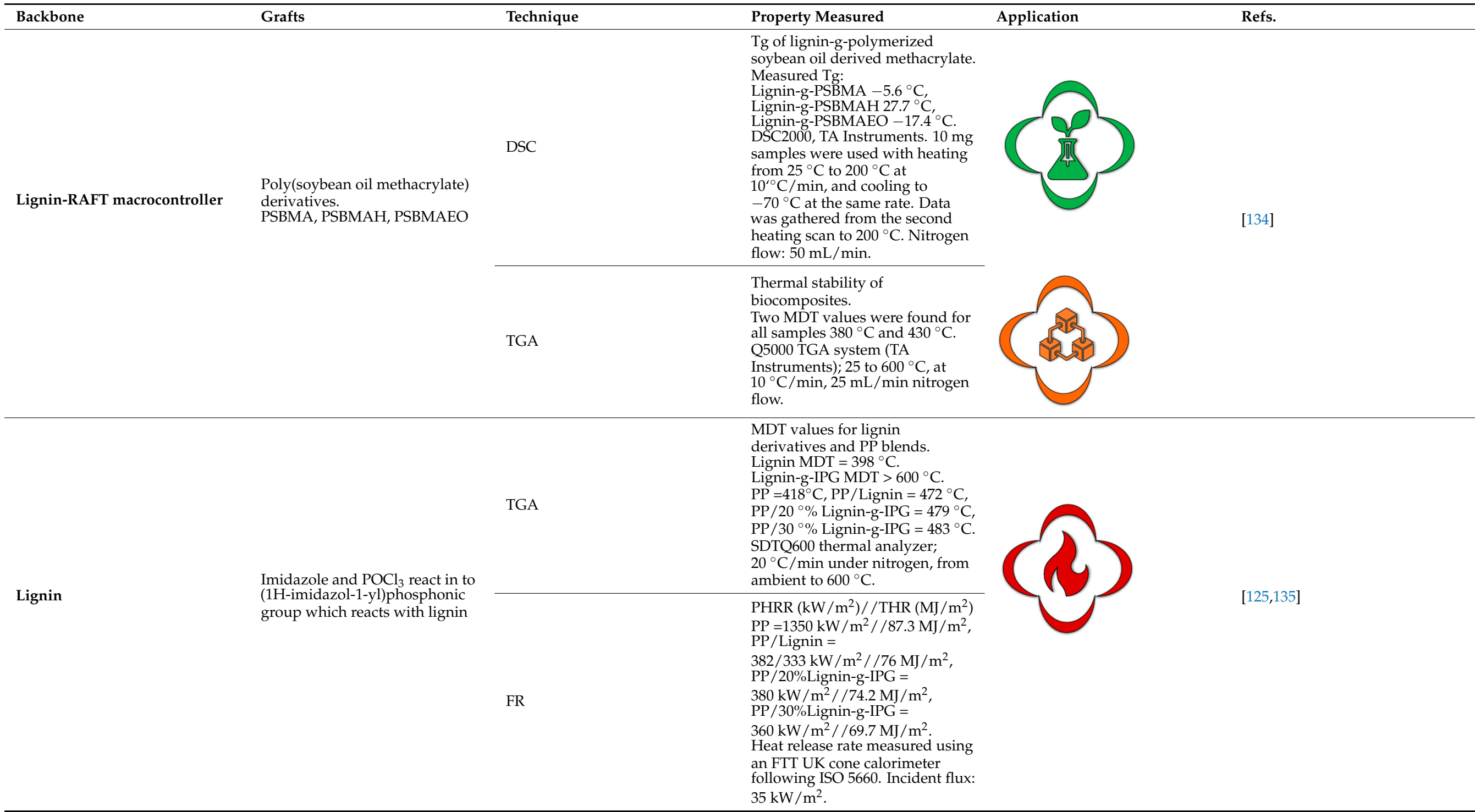


Table 7. Cont

\begin{tabular}{|c|c|c|c|c|c|}
\hline Backbone & Grafts & Technique & Property Measured & Application & Refs. \\
\hline \multirow{2}{*}{ Hydroxypropyl cellulose (HPC) } & \multirow{2}{*}{$\begin{array}{l}\text { Steglich esterification of PABTC } \\
\text { over HPC using DCC and DMAP } \\
\text { and grafting of PABTC and } \\
\text { further polymerization of EA } \\
\text { NIPAAM. }\end{array}$} & TGA & $\begin{array}{l}\text { TGA } 2950 \text { analyzer; nitrogen } \\
\text { atmosphere }\left(60 \mathrm{~cm}^{3} / \mathrm{min}\right) \text {. } \\
\text { Heating of samples }(5-10 \mathrm{mg}) \\
\text { from ambient temperature to } \\
600^{\circ} \mathrm{C} \text {, at } 10^{\circ} \mathrm{C} / \mathrm{min} \text {. } \\
\text { Calculations of the onset, the end } \\
\text { decomposition temperature, and } \\
\text { the residual mass. }\end{array}$ & & \multirow{2}{*}{ [108] } \\
\hline & & DSC & $\begin{array}{l}\text { Modulated DSC } 2920 \text { in nitrogen } \\
\text { environment }\left(60 \mathrm{~cm}^{3} / \mathrm{min}\right) \text {. The } \\
\text { determination of glass transition } \\
\text { temperature (Tg) required } \\
5-10 \mathrm{mg} \text { samples. Heating from } \\
\text { ambient temperature to } 200{ }^{\circ} \mathrm{C}, \\
\text { followed by cooling to }-100^{\circ} \mathrm{C}, \\
\text { and reheating to to } 200^{\circ} \mathrm{C} \text {, using } \\
10^{\circ} \mathrm{C} / \text { min for both, heating and } \\
\text { cooling was used. This sequence } \\
\text { occured three times. }\end{array}$ & & \\
\hline Cellulosic Grewia optiva fibers & $\begin{array}{l}\text { Redox initiator FAS-H2O2 for } \\
\text { grafting MA and KPS for } \\
\text { polymerization of MA. }\end{array}$ & TGA & $\begin{array}{l}\text { Thermo gravimetric analyses of } \\
\text { plain and grafted cellulosic fibers } \\
\text { carried out in presence of } \\
\text { nitrogen on a Perkin Elmer } \\
\text { thermal analyzer. Heating from } \\
\text { ambient temperature to } \\
900^{\circ} \mathrm{C} / \mathrm{min} \text {, at } 10^{\circ} \mathrm{C} / \mathrm{min} \text {. }\end{array}$ & & [110] \\
\hline Cotton linter cellulose & APS with MMA. & TGA-DTA & $\begin{array}{l}\text { Samples were analyzed using a } \\
\text { Pyris Diamond TG-DTA } \\
\text { (STA449C/3/F, Germany). } \\
\text { Sample weight: } 14.2 \mathrm{mg} \text {. } \\
\text { Heating rate: } 10^{\circ} \mathrm{C} / \mathrm{min} \text {. } \\
\text { Flow of nitrogen } 20 \mathrm{~mL} / \mathrm{min} \text {. }\end{array}$ & & [115] \\
\hline
\end{tabular}


Table 7. Cont

\begin{tabular}{|c|c|c|c|c|c|}
\hline Backbone & Grafts & Technique & Property Measured & Application & Refs. \\
\hline Cellulose (DP = 1130) & APS and MMA embedded. & TGA-DTA & $\begin{array}{l}\text { Samples analyzed in a Pyris } \\
\text { Diamond TG/DTA equipment } \\
\text { (STA449C/3/F, German). } \\
\text { Sample weight: } 14.2 \mathrm{mg} . \\
\text { Heating/Cooling rate: } \\
10^{\circ} \mathrm{C} / \mathrm{min} \text {. } \\
\text { Flow of nitrogen: } 20 \mathrm{~mL} / \mathrm{min} .\end{array}$ & & \\
\hline \multirow[t]{2}{*}{ Cellulose nanocrystal (CNC) } & \multirow{2}{*}{$\begin{array}{l}\text { Macromolecular initiator } \\
\text { obtained from the reaction } \\
\text { between Br-iBuBr and CNC with } \\
\text { TEA as catalyst via SI-ATRP with } \\
\text { sTY. Material was casted in } \\
\text { PMMA. }\end{array}$} & TGA & $\begin{array}{l}\text { Thermal degradation of studied } \\
\text { materials analyzed in a } \\
\text { TGA/SDTA } 851 \mathrm{e} \text { in nitrogen } \\
\text { atmosphere. Heating from } 30 \text { to } \\
550^{\circ} \mathrm{C} \text {, at } 10^{\circ} \mathrm{C} / \mathrm{min} \text {. }\end{array}$ & & \multirow{2}{*}{ [118] } \\
\hline & & DSC & $\begin{array}{l}\text { Tg determined in a Pyris } 1 \mathrm{DSC} \\
\text { equipment. Heating from } 30 \text { to } \\
200^{\circ} \mathrm{C} \text { at } 10^{\circ} \mathrm{C} / \mathrm{min} \text {. }\end{array}$ & & \\
\hline \multirow[b]{2}{*}{ Cellulose nanocrystal (CNC) } & \multirow[b]{2}{*}{$\begin{array}{l}\text { Macromolecular initiator } \\
\text { obtained from the reaction } \\
\text { between Br-iBuBr and CNC with } \\
\text { TEA as catalyst via SI-ATRP with } \\
\text { sTY. Material was casted in } \\
\text { PMMA. }\end{array}$} & TGA & $\begin{array}{l}\text { Analyses carried out in a TGA } \\
\text { Q50 (TA Instruments), under } \\
\text { nitrogen atmosphere } \\
(20 \mathrm{~mL} / \mathrm{min}) . \text { Heating from } 30 \text { to } \\
500^{\circ} \mathrm{C} \text { at } 5,10,15,20,25 \text { and } \\
30^{\circ} \mathrm{C} / \mathrm{min} \text { for determination of } \\
\text { degradation activation energy. }\end{array}$ & & \multirow[b]{2}{*}{ [119] } \\
\hline & & DSC & $\begin{array}{l}\text { Analyses performed in a Star } 1 \\
\text { (Mettler- Toledo). Heating from } \\
25^{\circ} \mathrm{C} \text { to } 200^{\circ} \mathrm{C} \text { at } 5^{\circ} \mathrm{C} / \mathrm{min} \text {, } \\
\text { remaining at } 200^{\circ} \mathrm{C} \text { for } 5 \mathrm{~min} \text {. } \\
\text { Cooling to }-50^{\circ} \mathrm{C} \text { at } 20^{\circ} \mathrm{C} / \mathrm{min} \text {, } \\
\text { remaining there for } 5 \mathrm{~min}, \\
\text { followed by heating to } 200^{\circ} \mathrm{C} \text { at } \\
5^{\circ} \mathrm{C} / \mathrm{min} \text {. Tg, Tm, Tc were } \\
\text { measured. Crystallinity (v) } \\
\text { calculated from melting enthalpy } \\
\text { of fully crystalline PLLA. }\end{array}$ & & \\
\hline Cellulose cotton fiber pulp & $\begin{array}{l}\text { Esterification of maleic } \\
\text { anhydride grafted over PHA (by } \\
\text { double bond). PHA-g-MA } \\
\text { grafted over cellulose cotton fiber } \\
\text { pulp (by the anhydride group). }\end{array}$ & TGA & $\begin{array}{l}\text { Analyses carried out on a Q500 } \\
\text { TGA (TA Instruments), in } \\
\text { nitrogen environment. Heating } \\
\text { from ambient to } 600^{\circ} \mathrm{C} \text { at } \\
20^{\circ} \mathrm{C} / \mathrm{min} \text {. }\end{array}$ & & [120] \\
\hline
\end{tabular}


Table 7. Cont.

\begin{tabular}{|c|c|c|c|c|c|}
\hline Backbone & Grafts & Technique & Property Measured & Application & Refs. \\
\hline Cellulosic filter papers & $\begin{array}{l}\text { Radiation-induced graft (RIGCP) } \\
\text { copolymerization of acrylonitrile. }\end{array}$ & TGA & $\begin{array}{l}\text { Analyses obtained in TGA-50 } \\
\text { (Shimadzu). } \\
\text { Heating rate: } 10^{\circ} \mathrm{C} / \mathrm{min} . \mathrm{N}_{2} \\
\text { environment. }\end{array}$ & & [121] \\
\hline \multirow[t]{2}{*}{ Hemicellulose } & \multirow[t]{2}{*}{$\begin{array}{l}\text { Hemicellulose grafting using } \\
\text { TBD and e-caprolactona } \\
\text { monomer }\end{array}$} & TGA & $\begin{array}{l}\text { TGA/DSC } 1 \text { STARe System } \\
\text { (Mettler Toledo). } 5-15 \mathrm{mg} \text { of a } \\
\text { sample. Nitrogen environment } \\
\text { ( } 85 \mathrm{~mL} / \mathrm{min} \text { ). } \\
\text { Heating from } 30-500{ }^{\circ} \mathrm{C} \text { at } \\
10^{\circ} \mathrm{C} / \text { min. } \\
\text { Compositions of produced gases } \\
\text { measured in an FTIR (TA, } \\
\text { Nicolet, iS); detector coupled to } \\
\text { TGA. FTIR analyses from } 4000 \text { to } \\
650 \mathrm{~cm}^{-1} \text { with a } 4 \mathrm{~cm}^{-1} \\
\text { resolution. }\end{array}$ & & \multirow[t]{2}{*}{ [37] } \\
\hline & & DSC & $\begin{array}{l}\text { DSCQ10 (TA Instruments). } \\
\text { Hermetic pan (T } 090127) \text {. } \\
\text { Heating from } 25 \text { to } 165^{\circ} \mathrm{C} \text { at } 10 \\
{ }^{\circ} \mathrm{C} / \text { min. Cooling to } 15^{\circ} \mathrm{C} \\
\text { followed by heating to } 165^{\circ} \mathrm{C} \text { at } \\
10{ }^{\circ} \mathrm{C} / \text { min. } \\
\text { Tg obtained from second heating } \\
\text { ramp. }\end{array}$ & & \\
\hline
\end{tabular}


Table 7. Cont

\begin{tabular}{|c|c|c|c|c|c|}
\hline Backbone & Grafts & Technique & Property Measured & Application & Refs. \\
\hline CellClAc & $\begin{array}{l}\text { NCHA, } 4 \text { VP, DA and DAAM } \\
\text { grafted by atom transfer radical } \\
\text { polymerization (ATRP) using } \\
\mathrm{CuCl}, 2^{\prime} 2^{\prime} \text { BIPI as catalyst. }\end{array}$ & TGA & $\begin{array}{l}\text { Thermal analyses carried out in a } \\
\text { Shimadzu TGA-50 at } 10^{\circ} \mathrm{C} / \mathrm{min} \\
\text { in nitrogen environment } \\
(10 \mathrm{~mL} / \mathrm{min}) \text {. } \\
\text { Cell-g-DA and Cell-g- } 4 \mathrm{VP} \\
\text { exhibited higher decomposition } \\
\text { temperatures. }\end{array}$ & & [122] \\
\hline Chitosan & $\begin{array}{l}\text { Embedded polymerization N-VP, } \\
\text { 4VP. Crosslinking agent: } \\
\text { N'N'-MBA }\end{array}$ & TGA & $\begin{array}{l}\text { TGA analyses carried out using a } \\
\text { NETZSCH, STA } 409 \text { PG.4.G Lux. } \\
50 \mathrm{~mL} / \mathrm{min} \text { nitrogen } \\
\text { environment. } \\
\text { Heating from } 20^{\circ} \mathrm{C} \text { to } 400^{\circ} \mathrm{C} \text { at } \\
10^{\circ} \mathrm{C} / \mathrm{min} \text {. }\end{array}$ & & [164] \\
\hline
\end{tabular}


Table 8. Summary of spectroscopic characterization techniques employed in grafted materials.

\begin{tabular}{|c|c|c|c|c|c|}
\hline Backbone & Grafts & Technique & Property Measured & Application & Refs. \\
\hline \multirow[t]{2}{*}{ Lignin-RAFT macrocontroller } & \multirow[t]{2}{*}{$\begin{array}{l}\text { Poly(soybean oil methacrylate) } \\
\text { derivatives. } \\
\text { PSBMA, PSBMAH, PSBMAEO. }\end{array}$} & ${ }^{1} \mathrm{H}-\mathrm{NMR}$ & $\begin{array}{l}\text { Amount of RAFT controller } \\
\text { reacted (shifts } 7.3,7.6,7.9 \mathrm{ppm} \text { ) } \\
\text { and nonattached monomer } \\
\text { (shifts } 5.3,5.7 \mathrm{ppm} \text { ). } \\
\text { Varian Mercury } 300 \text { spectrometer, } \\
300 \mathrm{MHz} \text { t tetramethyl silane as } \\
\text { an internal reference. }\end{array}$ & & \multirow[t]{2}{*}{ [134] } \\
\hline & & FTIR & $\begin{array}{l}\text { Amount of RAFT controller and } \\
\text { polymer attached to lignin. Area } \\
\text { of peak at } 1760 \mathrm{~cm}^{-1} \text {. } \\
\text { Perkin Elmer spectrum } 100 \text {. ATR } \\
\text { method, recorded at } 4 \mathrm{~cm}^{-1} \text {, } \\
32 \text { scans. }\end{array}$ & & \\
\hline \multirow[b]{2}{*}{ Lignin } & \multirow[b]{2}{*}{$\begin{array}{l}\text { Imidazole and } \mathrm{POCl}_{3} \text { react in to } \\
\text { (1H-imidazol-1-yl) phosphonic } \\
\text { group which reacts with lignin. }\end{array}$} & FTIR & $\begin{array}{l}\text { Lignin, lignin-OH, imidazole and } \\
\text { lignin-g-IPG were compared at } \\
\text { relevant bands }(1662,1260,1080, \\
\left.\text { 1050, } 878 \text {, and } 791 \mathrm{~cm}^{-1}\right) \text { for } \\
\text { determination of } \mathrm{C}=\mathrm{N}, \mathrm{P}=\mathrm{O}, \\
\mathrm{P}-\mathrm{O}-\mathrm{C}, \mathrm{P}-\mathrm{O},-\mathrm{OH} \text {, and } \mathrm{P}-\mathrm{N} \\
\text { groups, respectively. A Bruker } \\
\text { VECTOR 22 spectrometer } \\
\text { was used. }\end{array}$ & & \multirow[b]{2}{*}[125,135]{} \\
\hline & & XPS & $\begin{array}{l}\text { Surface elementary composition } \\
\text { of samples (wt.\%), were } \\
\text { compared to determine the } \\
\text { amount of functionalized or } \\
\text { grafted groups in the surface. } \\
\text { Elements analyzed: carbon, } \\
\text { oxygen, phosphorous, nitrogen. } \\
\text { Lignin, C: } 68.2, \text { O: } 31.9 \text {; Modified } \\
\text { lignin-OH, C: 67.5, O: } 32.5 ; \\
\text { Lignin-g-IPG, C: } 53.0, \text { O: } 31.7, \text { P: } \\
\text { 8.1, N: 7.2. } \\
\text { A Thermo ESCALAB } 250 \\
\text { spectrometer was used. Analyses } \\
\text { were carried out from } 50 \text { up to } \\
800 \text { eV of binding energy. }\end{array}$ & & \\
\hline
\end{tabular}


Table 8. Cont.

\begin{tabular}{|c|c|c|c|c|c|}
\hline Backbone & Grafts & Technique & Property Measured & Application & Refs. \\
\hline Hydroxypropyl cellulose (HPC) & $\begin{array}{l}\text { Steglich esterification of PABTC } \\
\text { over HPC using DCC and } \\
\text { DMAP; grafting of PABTC and } \\
\text { further polymerization of EA } \\
\text { NIPAAM. }\end{array}$ & ${ }^{1} \mathrm{H} \mathrm{NMR}$ & $\begin{array}{l}\text { NMR spectra obtained using a } \\
\text { Bruker } 300 \text { or } 200 \mathrm{MHz} \text { in } \\
\text { deuterated chloroform or } \\
\text { deuterated dimethyl sulfoxide } \\
\text { (d6-DMSO). }\end{array}$ & & [108] \\
\hline \multirow{2}{*}{ CellClAc } & \multirow{2}{*}{$\begin{array}{l}\text { Macro initiator, } \mathrm{Cu}(\mathrm{I}) \mathrm{Cl} / 2^{\prime} 2^{\prime} \mathrm{BIPI} \\
\text { catalytic system via ATRP of } \\
4 \mathrm{NPA} \text { and MMA }\end{array}$} & ${ }^{1} \mathrm{H}-\mathrm{NMR}$ & $\begin{array}{l}\text { NMR spectra obtained using a } \\
\text { Bruker } 400 \mathrm{MHz} \\
\text { spectrometer at room } \\
\text { temperature in DMSO- } \mathrm{d}_{6} \text {. }\end{array}$ & & \multirow[b]{2}{*}{ [109] } \\
\hline & & FTIR & $\begin{array}{l}\text { FTIR data using solid samples as } \\
\mathrm{KBr} \text { pellets, from } 4000 \mathrm{~cm}^{-1} \text { to } \\
450 \mathrm{~cm}^{-1} \text {. }\end{array}$ & & \\
\hline Cellulosic Grewia optiva fibers & $\begin{array}{l}\text { Redox initiator FAS- } \mathrm{H}_{2} \mathrm{O}_{2} \text { for } \\
\text { grafting of MA, and KPS for } \\
\text { polymerization of MA. }\end{array}$ & FTIR & $\begin{array}{l}\text { Chemical structures of fibers } \\
\text { before and after grafting were } \\
\text { studied using FTIR (PERKIN } \\
\text { ELMER RXI). Spectrum recorded } \\
\text { from } 4000 \text { to } 400 \mathrm{~cm}^{-1} \text {. }\end{array}$ & & [110] \\
\hline \multirow{2}{*}{ Cellulose acetate } & \multirow{2}{*}{$\begin{array}{l}\text { Solvents: DMSO, PDX, DMAc, } \\
\mathrm{C}_{3} \mathrm{H}_{6} \mathrm{O} \text {. } \\
\text { Initiators for grafting and } \\
\text { polymerization: CAN, } \mathrm{Sn}(\mathrm{Oct})_{2} \\
\text { and BPO. }\end{array}$} & ${ }^{1} \mathrm{H}-\mathrm{NMR}$ & $\begin{array}{l}\text { Spectra of studied materials } \\
\text { obtained using a Bruker WM- } 400 \\
\text { apparatus, at } 300 \mathrm{MHz} \\
\text { Tetramethyl silane (TMS) used as } \\
\text { internal standard and DMSO-d6 } \\
\text { as solvent. }\end{array}$ & & \multirow{2}{*}{ [111] } \\
\hline & & FTIR & $\begin{array}{l}\text { Grafted and ungrafted materials } \\
\text { analyzed using a Spectrum RX1 } \\
\text { PerkinElmer apparatus. } \\
\text { Chloroform used as a solvent. } \\
\text { KBr pellet technique used for } \\
\text { grafted materials. }\end{array}$ & & \\
\hline
\end{tabular}


Table 8. Cont.

\begin{tabular}{|c|c|c|c|c|c|}
\hline Backbone & Grafts & Technique & Property Measured & Application & Refs. \\
\hline CellClAc & $\begin{array}{l}\text { Macro initiator, } \mathrm{Cu}(\mathrm{I}) \mathrm{Cl} / 2^{\prime} 2^{\prime} \mathrm{BIPI} \\
\text { catalytic system via ATRP } \\
\text { controller. }\end{array}$ & FTIR & $\begin{array}{l}\text { FTIR spectrophotometer on solid } \\
\text { samples as KBr pellets, from } \\
4000 \mathrm{~cm}^{-1} \text { to } 450 \mathrm{~cm}^{-1} \text {. }\end{array}$ & & [112] \\
\hline Cellulose cotton fibers & $\begin{array}{l}\mathrm{Na}_{2} \mathrm{CO}_{3} \text { and thermal activation } \\
\text { with MTC-b-CD and silver } \\
\text { nitrate. }\end{array}$ & FTIR & $\begin{array}{l}\text { MCT- } \beta \text {-CD grafted and } \\
\text { ungrafted cotton fabrics were } \\
\text { studied with attenuated total } \\
\text { reflection (FTIR-ATR), which } \\
\text { permits the study of solid } \\
\text { surfaces using a diamond tipped } \\
\text { device (SPECAC), connected to a } \\
\text { Bruker Vertex 70 } \\
\text { spectrophotometer. }\end{array}$ & & [113] \\
\hline \multirow[b]{2}{*}{ Cotton linter cellulose } & \multirow[b]{2}{*}{ APS with MMA. } & FTIR & $\begin{array}{l}\text { A TENSOR27 FTIR apparatus } \\
\left(4500-400 \mathrm{~cm}^{-1}\right) \text { was used to } \\
\text { analyze materials. }\end{array}$ & & \multirow[b]{2}{*}{ [115] } \\
\hline & & XRD & $\begin{array}{l}\text { X-ray diffractometer } \\
(\mathrm{D} / \mathrm{max}-2500 / \mathrm{PC} \text {, Rigaku Co) } \\
\text { based on a reflection method } \\
\text { using a Cu Ka target ( } 40 \mathrm{kV} \text { and } \\
60 \mathrm{~mA} \text { ). Diffraction angle: } 5^{\circ} \text { to } \\
60^{\circ} \text {. }\end{array}$ & & \\
\hline
\end{tabular}


Table 8. Cont.

\begin{tabular}{|c|c|c|c|c|c|}
\hline Backbone & Grafts & Technique & Property Measured & Application & Refs. \\
\hline \multirow{3}{*}{ Microcrystalline cellulose } & \multirow{3}{*}{$\begin{array}{l}\text { Ring-opening polymerization } \\
\text { (ROP) of L-LA with DMAP in an } \\
\text { ionic liquid AmimCl to form } \\
\text { Cellulose- } g \text {-PLLA }\end{array}$} & ${ }^{1} \mathrm{H}-\mathrm{NMR}$ & $\begin{array}{l}\text { Materials analyzed using } \\
{ }^{1} \mathrm{H}-\mathrm{NMR} \text { (Bruker AV400-MHz). } \\
\text { Solvent: DMSO-d6 with a drop of } \\
\text { trifluoroacetic acid-d; internal } \\
\text { standard: tetramethyl silane } \\
\text { (TMS). }\end{array}$ & & \multirow{3}{*}{ [116] } \\
\hline & & $\begin{array}{l}\text { XRD } \\
\text { WAXD }\end{array}$ & $\begin{array}{l}\text { WAXD carried out using an } \\
\text { XRD-6000 X-ray diffractometer } \\
\text { (Shimadzu, Japan); Ni-filtered Cu } \\
\text { Ka radiation ( } 40 \mathrm{kV}, 30 \mathrm{~mA}) ; \\
4^{\circ} / \text { min at ambient temperature. }\end{array}$ & & \\
\hline & & UV spectroscopy & $\begin{array}{l}\text { UV analyses performed in a } \\
\text { UV2000 equipment (UNICO, } \\
\text { China), to follow the load and } \\
\text { controlled release of vitamin C. }\end{array}$ & & \\
\hline \multirow[b]{2}{*}{ Cellulose (DP = 1130) } & \multirow[b]{2}{*}{ APS and MMA embedded. } & FTIR & $\begin{array}{l}\text { A TENSOR27 FTIR apparatus } \\
\left(4500-400 \mathrm{~cm}^{-1}\right) \text { was used to } \\
\text { analyze materials. }\end{array}$ & & \multirow[b]{2}{*}{ [117] } \\
\hline & & WAXD-XRD & $\begin{array}{l}\text { WAXD carried out in an X-ray } \\
\text { diffractometer (D/max-2500/PC, } \\
\text { Rigaku Co. Ltd., Tokyo); Cu Ka } \\
\text { radiation }(40 \mathrm{kV}, 60 \mathrm{~mA}) ; \\
2 \mathrm{q}=5^{\circ}-60^{\circ} \text {. }\end{array}$ & & \\
\hline \multirow{4}{*}{ Cellulose nanocrystal (CNC) } & \multirow{4}{*}{$\begin{array}{l}\text { Macromolecular initiator } \\
\text { obtained from the reaction } \\
\text { between Br-iBuBr and CNC with } \\
\text { TEA as catalyst via SI-ATRP with } \\
\text { STy. Material was casted in } \\
\text { PMMA. }\end{array}$} & FTIR & $\begin{array}{l}\text { Spectra obtained at ambient } \\
\text { temperature on a NICOLET } \\
\text { spectrometer } 10 \text { to study surface } \\
\text { modification of studied materials. } \\
\text { KBr pellet method used for } \\
\text { samples. Resolution: } 4 \mathrm{~cm}^{-1} \text {; } \\
\text { measurement range; } \\
4000-400 \mathrm{~cm}^{-1} \text {, and } 30 \text { scans. }\end{array}$ & & \multirow{4}{*}{ [118] } \\
\hline & & UV-Vis & $\begin{array}{l}\text { Transmittance of samples } \\
\text { measured using a UV-vis } \\
\text { spectrophotometer at } \\
\text { wavelengths of } 400 \text { to } 800 \mathrm{~nm} \text {. }\end{array}$ & & \\
\hline & & XRD & $\begin{array}{l}\text { XRD patterns of studied } \\
\text { materials were obtained using a } \\
\text { Bruker Siemens D8 X-ray } \\
\text { apparatus with operation at } 3 \\
\mathrm{~kW} ; \text { CuKa radiation }(1= \\
0.154 \mathrm{~nm}) ; 2 \mathrm{q}=3-60^{\circ} ; 0.02^{\circ} \text { step. }\end{array}$ & & \\
\hline & & ${ }^{13} \mathrm{C}-\mathrm{NMR}$ & $\begin{array}{l}\text { Chemical structures of studied } \\
\text { materials obtained with the help } \\
\text { of a Bruker } 400 \mathrm{M} \text { solid-state } \\
{ }^{13} \text { C-NMR apparatus. }\end{array}$ & & \\
\hline
\end{tabular}


Table 8. Cont.

\begin{tabular}{|c|c|c|c|c|c|}
\hline Backbone & Grafts & Technique & Property Measured & Application & Refs. \\
\hline \multirow{4}{*}{ Cellulose nanocrystal (CNC) } & \multirow{4}{*}{$\begin{array}{l}\text { Redox agent: } \mathrm{MgH}_{2} . \\
\text { L-LA polymerization in situ. } \\
\text { PLLA-g-CNC particles were } \\
\text { casted in PLLA. }\end{array}$} & FTIR & $\begin{array}{l}\text { A Perkin Elmer Spectrum } 100 \\
\text { apparatus in ATR mode, } \\
\text { equipped with a Zn-Se crystal, } \\
\text { was used. Modified and } \\
\text { unmodified CNC samples. } \\
\text { Range: } 4000 \text { to } 650 \mathrm{~cm}^{-1} \text {. } \\
\text { Accuracy: } 4 \mathrm{~cm}^{-1} \text { for } 64 \text { scans. }\end{array}$ & & \multirow{4}{*}{ [119] } \\
\hline & & $\begin{array}{l}{ }^{1} \mathrm{H}-\mathrm{NMR} \\
{ }^{13} \mathrm{C}-\mathrm{NMR}\end{array}$ & $\begin{array}{l}\text { A Bruker-300 apparatus with } \\
\text { DMSO-d6 as solvent was used. } \\
\text { Small amounts of CDCl } \mathrm{CD}_{3} \text { were } \\
\text { used to improve solubility of } \\
\text { grafted product in DMSO-d6. }\end{array}$ & & \\
\hline & & XRD & $\begin{array}{l}\text { XRD analyses carried out in a } \\
\text { PAN analytical apparatus; Cu Ka } \\
\text { radiation }(1=1.541 \AA \AA \text { ); incidence } \\
\text { angle: } 10 \text { to } 40^{\circ}, 0.07^{\circ} \text { steps. }\end{array}$ & & \\
\hline & & XPS & $\begin{array}{l}\text { XPS analyses performed in an } \\
\text { ESCALAB } 250 \text { equipment } \\
\text { (Thermo Scientific). Analyzed } \\
\text { surface: } 400 \mathrm{~mm}^{2} \text {. Atomic } \\
\text { concentrations estimated from } \\
\text { areas of photoelectron peaks } \\
\text { considering atomic sensitivity } \\
\text { factors. All binding energies } \\
\text { were charge-corrected to } 284.8 \mathrm{eV} \text {. }\end{array}$ & & \\
\hline \multirow[b]{2}{*}{ Cellulose cotton fiber pulp } & \multirow{2}{*}{$\begin{array}{l}\text { Esterification of maleic } \\
\text { anhydride grafted onto PHA } \\
\text { (through double bond). } \\
\text { PHA-g-MA grafted onto } \\
\text { cellulose cotton fiber pulp } \\
\text { (through the anhydride group). }\end{array}$} & FTIR & $\begin{array}{l}\text { ATR-FTIR analyses of ungrafted } \\
\text { and grafted materials acquired } \\
\text { using } \mathrm{KBr} \text { discs on a Vector } 33 \\
\text { Bruker apparatus. }\end{array}$ & & \multirow[b]{2}{*}{ [120] } \\
\hline & & XRD & $\begin{array}{l}\text { XRD analyses carried out in a } \\
\text { Bruker D8 ADVANCE X-ray } \\
\text { apparatus with operation at } 40 \\
\text { mA and } 40 \mathrm{kV} \text {. Cu K } \alpha \text { filtered } \\
\text { radiation }(\lambda=0.15418 \mathrm{~nm}) \text {. } \\
\text { Scattering angle }(2 \theta): 5^{\circ} \text { to } 50^{\circ} \text {, } \\
0.02^{\circ} \text { step. }\end{array}$ & & \\
\hline
\end{tabular}


Table 8. Cont.

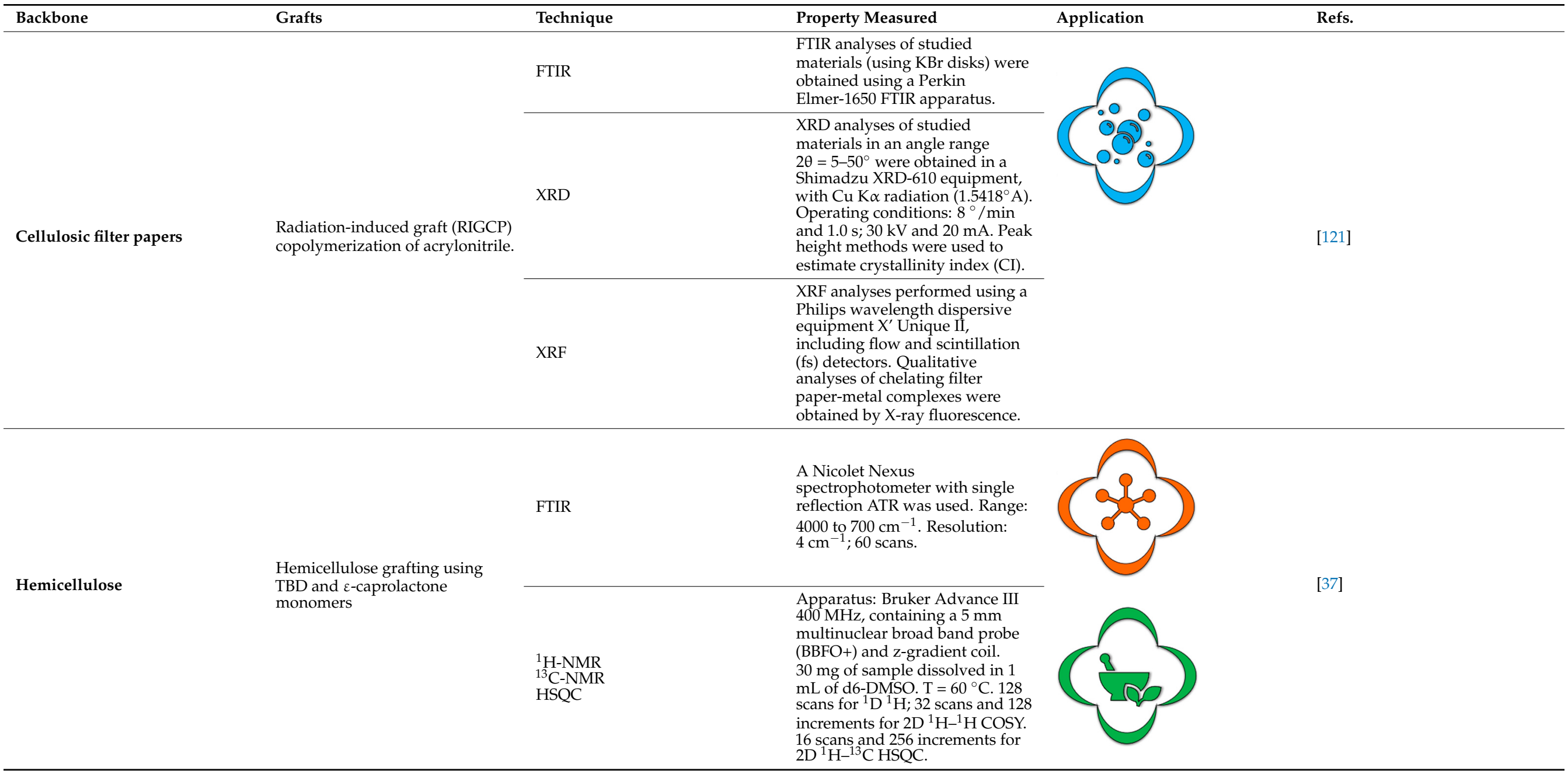


Table 8. Cont.

\begin{tabular}{|c|c|c|c|c|c|}
\hline Backbone & Grafts & Technique & Property Measured & Application & Refs. \\
\hline \multirow{3}{*}{ CellClAc } & \multirow{3}{*}{$\begin{array}{l}\text { NCHA, } 4 \text { VP, DA and DAAM } \\
\text { grafted by ATRP using CuCl, } \\
2^{\prime} 2^{\prime} \text { BIPI as catalyst. }\end{array}$} & FTIR & $\begin{array}{l}\text { Apparatus: Perkin Elmer } \\
\text { Spectrum One. Solid samples as } \\
\text { KBr pellets. }\end{array}$ & & \multirow{3}{*}{ [122] } \\
\hline & & & & & \\
\hline & & UV-Visible & $\begin{array}{l}\text { Apparatus: Shimadzu } 3600 \\
\text { UV-VIR-NIR. }\end{array}$ & & \\
\hline \multirow[b]{2}{*}{ Chitosan } & \multirow{2}{*}{$\begin{array}{l}\text { Embedded polymerizations of } \\
\text { N-VP and 4VP. Crosslinking } \\
\text { agent: N'N'-MBA }\end{array}$} & FTIR & $\begin{array}{l}\text { Apparatus: AVATAR } 370 \text { Thermo } \\
\text { Nicolet. Range: } 4000 \text { to } 500 \mathrm{~cm}^{-1} \text {, } \\
\text { using the KBr pellet method. } \\
\text { Spectral resolution: } 4 \mathrm{~cm}^{-1} \text {. }\end{array}$ & & \multirow[b]{2}{*}{ [164] } \\
\hline & & XRD & $\begin{array}{l}\text { Apparatus: Bruker Advance D8 } \\
\text { equipment (Germany). } \\
\text { Diffractograms comprised ( } 2 \theta) \\
0.020 \text { imaging; scattering rates: } 3 \\
\text { to } 800 \text {; scanning speeds: } \\
2.0 \mathrm{~min}^{-1} \text {; accelerated tension of } \\
40 \mathrm{kV} \text {; intensity of } 35 \mathrm{~mA} \text {. }\end{array}$ & & \\
\hline \multirow{3}{*}{ Cellulose nanofibrils } & \multirow{3}{*}{$\begin{array}{l}\text { Nitroxide TEMPO insertion and } \\
\text { nitroxide mediated } \\
\text { polymerization of HEMA }\end{array}$} & XRD & $\begin{array}{l}\text { XRD using a Bruker D8 Advance } \\
\text { spectrometer. Analyses carried } \\
\text { out from } 5^{\circ} \text { to } 40^{\circ} \text { at } 4^{\circ} / \mathrm{min}, \\
\text { with a current of } 40 \mathrm{~mA} \text {. } \\
\text { Measured property: CI. }\end{array}$ & & \multirow{3}{*}{ [124] } \\
\hline & & FTIR & $\begin{array}{l}\text { Apparatus: Bruker spectrometer, } \\
\text { accumulation of } 128 \text { scans. } \\
\text { Resolution: } 4 \mathrm{~cm}^{-1} \text {. Range: } \\
4000-400 \mathrm{~cm}^{-1} \text {; absorbance } \\
\text { mode. }\end{array}$ & & \\
\hline & & XPS & $\begin{array}{l}\text { XPS analyses using Thermo } \\
\text { Electron Scientific Instruments } \\
\text { were performed using a } \\
1486.6 \mathrm{eV} \mathrm{Al} \mathrm{K \alpha} \text { X-ray source. }\end{array}$ & & \\
\hline
\end{tabular}


Table 8. Cont.

\section{Backbone}

Lignin
Grafts

Technique

Dynamic light scattering (Particle

size distribution)

Insertion of ACX onto lignin to

get a RAFT macrocontroller able

to polymerize AM and AA.

${ }^{1} \mathrm{H}-\mathrm{NMR}$
Property Measured

Application

Refs.

Particle size distributions (PSD)

measured in an aqueous solution

$(1 \mathrm{mg} / \mathrm{mL})$ using a DLS

Zeta-sizer (Malvern Instruments)

Apparatus: Bruker 300 Advance,

PFB used as internal standard to

ransform proton intensities into

initiator concentration $(\mu \mathrm{mol} /(\mathrm{g}$

lignin))

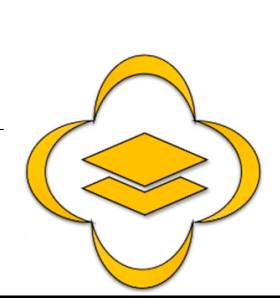


Table 9. Summary of digital imaging and microscopy characterization techniques employed in grafted materials.

\begin{tabular}{|c|c|c|c|c|c|}
\hline Backbone & Grafts & Technique & Property Measured & Application & Refs. \\
\hline \multirow[t]{2}{*}{ Lignin } & \multirow[t]{2}{*}{$\begin{array}{l}\text { Imidazole and } \mathrm{POCl}_{3} \text { react to } \\
\text { produce }(1 \mathrm{H} \text {-imidazol-1-yl) } \\
\text { phosphonic, which reacts } \\
\text { with lignin. }\end{array}$} & SEM with EDAX & $\begin{array}{l}\text { S4800 FEI SEM; } 5 \mathrm{kV} \text { accelerating voltage. Comparison of } \\
\text { morphologies of char residues after cone calorimeter } \\
\text { analyses. PP/Lignin displayed loosely spheroidal } \\
\text { structures with C: } 86.5 \mathrm{wt} . \%, \mathrm{O}: 13.5 \mathrm{wt} . \% \text {. PP } / 30 \% \% \\
\text { Lignin-g-IPG formed a continuous and compact spheroidal } \\
\text { structure. }\end{array}$ & & \multirow[t]{2}{*}[125,135]{} \\
\hline & & Digital photo & $\begin{array}{l}\text { Digital photos comparing PP composite sample test probes } \\
\text { after cone calorimeter measurements were obtained. }\end{array}$ & & \\
\hline $\begin{array}{l}\text { Cellulosic Grewia optiva } \\
\text { fibers }\end{array}$ & $\begin{array}{l}\text { FAS- } \mathrm{H}_{2} \mathrm{O}_{2} \text { redox initiation for } \\
\text { grafting of MA and KPS, for } \\
\text { polymerization of MA. }\end{array}$ & SEM. & $\begin{array}{l}\text { The morphologies of ungrafted and grafted fibers were } \\
\text { studied using a SEM apparatus (LEO } 435 \mathrm{VP} \text { ). }\end{array}$ & & [110] \\
\hline $\begin{array}{l}\text { Microcrystalline } \\
\text { cellulose }\end{array}$ & $\begin{array}{l}\text { Ring-opening polymerization } \\
\text { (ROP) of L-LA using DMAP } \\
\text { in an ionic liquid (AmimCl) } \\
\text { to produce Cellulose- } g \text {-PLLA }\end{array}$ & TEM & $\begin{array}{l}\text { Apparatus: JEM-100CXa TEM at an acceleration voltage of } \\
100 \mathrm{kV} . . \text { Sample preparation involved dissolving them in } \\
\text { DMSO } 0.01 \%(w / v) \text { and placement on a copper grid with } \\
\text { formvar film. }\end{array}$ & & [116] \\
\hline
\end{tabular}


Table 9. Cont

\begin{tabular}{|c|c|c|c|c|c|}
\hline Backbone & Grafts & Technique & Property Measured & Application & Refs. \\
\hline Cellulose (DP = 1130) & Embedded APS and MMA. & SEM & $\begin{array}{l}\text { Apparatus: JSM- } 6700 \mathrm{~F} \text { scanning microscope. Coating with } \\
\text { a thin layer of gold required for samples. }\end{array}$ & & [117] \\
\hline \multirow[t]{2}{*}{$\begin{array}{l}\text { Cellulose nanocrystal } \\
\text { (CNC) }\end{array}$} & \multirow{2}{*}{$\begin{array}{l}\text { Macromolecular initiator } \\
\text { obtained from Br-iBuBr and } \\
\text { CNC with TEA as catalyst via } \\
\text { SI-ATRP with Sty. Material } \\
\text { was casted in PMMA. }\end{array}$} & SEM & $\begin{array}{l}\text { Apparatus: su1510 (Hitachi Zosen Corporation) operating } \\
\text { at } 30 \mathrm{kV} \text {. Focus: measurement of homogeneity of PMMA } \\
\text { composites. }\end{array}$ & & \multirow[t]{2}{*}{ [118] } \\
\hline & & TEM & $\begin{array}{l}\text { Apparatus: JEM- } 2100 \text { electron microscope. Operation } \\
\text { involves acceleration voltage of } 200 \mathrm{kV} \text {. Focus: Analysis of } \\
\text { morphological features and distribution of cellulose } \\
\text { nanocrystals. }\end{array}$ & & \\
\hline $\begin{array}{l}\text { Cellulose cotton fiber } \\
\text { pulp }\end{array}$ & $\begin{array}{l}\text { Esterification of maleic } \\
\text { anhydride grafted onto PHA } \\
\text { (through double bond). } \\
\text { PHA-g-MA grafted onto } \\
\text { cellulose cotton fiber pulp } \\
\text { (through the anhydride } \\
\text { group). }\end{array}$ & SEM & $\begin{array}{l}\text { Apparatus: Nova Nano SEM } 430 \text { (FEI Company); } \\
\text { high-resolution field emission with operation at an } \\
\text { acceleration voltage of } 15 \mathrm{kV} \text {. Focus: Morphological } \\
\text { analysis of PHA/CF composite films. }\end{array}$ & & [108] \\
\hline Cellulosic filter papers & $\begin{array}{l}\text { Radiation-induced graft } \\
\text { (RIGCP) copolymerization of } \\
\text { acrylonitrile. }\end{array}$ & SEM & $\begin{array}{l}\text { SEM micrographs of samples were obtained on a } \\
\text { JEOL-SEM } 5400 \text { microscope. }\end{array}$ & & [109] \\
\hline Chitosan & $\begin{array}{l}\text { Embedded polymerization of } \\
\text { N-VP and } 4 \mathrm{VP} \text {; Crosslinking } \\
\text { agent: } \mathrm{N}^{\prime} \mathrm{N}^{\prime}-\mathrm{MBA}\end{array}$ & SEM & $\begin{array}{l}\text { Morphological surface images of ungrafted and grafted } \\
\text { chitosan with or without doxocyline were obtained using a } \\
\text { JSM- } 6390 \text { SEM microscope. }\end{array}$ & & [153] \\
\hline
\end{tabular}


Table 9. Cont.

Regenerated cellulose fibres (rayon)

Photo-chemical grafting of PETA without photoinitiator.

Apparatus: JSM-6510 instrument (Joel GmbH, Freising DE). Samples prepared following standard procedures. Micrographs obtained in SE mode; acceleration voltage: 5 and $10 \mathrm{kV}$. Purpose: Analyses of qualitative fracture.

SEM

Apparatus: Hitachi S-4800 SEM microscope. Purpose:

Nitroxide TEMPO insertion and NMP of HEMA
SEM
Analysis of morphologies of materials at working distances of $15 \mathrm{~mm}$. Operating voltage: of $2 \mathrm{kV}$.
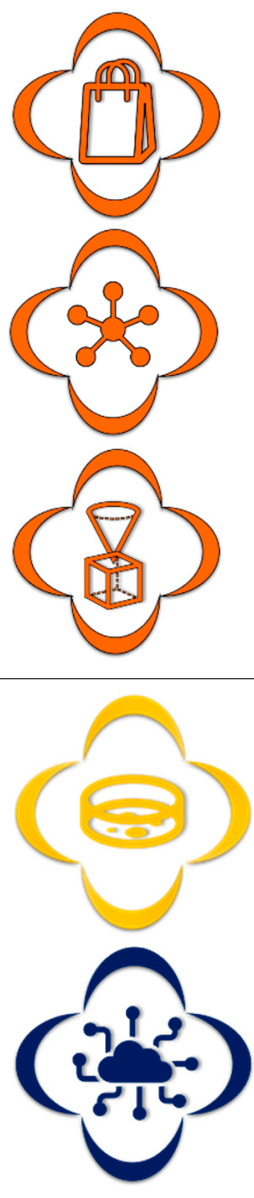
Table 10. Summary of rheological characterization techniques used for grafted materials.

\begin{tabular}{|c|c|c|c|c|c|}
\hline Backbone & Grafts & Technique & Property Measured & Application & Refs. \\
\hline \multirow{2}{*}{$\begin{array}{l}\text { Cellulose nanocrystal } \\
\text { (CNC) }\end{array}$} & \multirow{2}{*}{$\begin{array}{l}\text { Redox agent: } \mathrm{MgH}_{2} ; \text { in situ } \\
\text { L-LA polymerization. } \\
\text { PLLA-g-CNC particles were } \\
\text { casted in PLLA. }\end{array}$} & $\begin{array}{l}\text { DMA. Casted } \\
\text { PLLA-g-CNC particles } \\
\text { in PLLA matrix. }\end{array}$ & $\begin{array}{l}\text { Apparatus: DMA } 50 \text {, Metravib, tensile mode. Dimensions } \\
\text { of sample specimens: } 6.5 \times 10.0 \times 0.2 \mathrm{~mm} \text {. Storage } \mathrm{E}^{\prime} \text { and } \\
\text { loss E" moduli were registered. Heating rate: } 5^{\circ} \mathrm{C} / \mathrm{min} \text {; } \\
-100-200^{\circ} \mathrm{C} ; 1 \mathrm{~Hz} \text {. }\end{array}$ & & \multirow{2}{*}{ [119] } \\
\hline & & $\begin{array}{l}\text { Molten Rheometry; } \\
\text { Casted PLLA-g-CNC } \\
\text { particles in PLLA } \\
\text { matrix. }\end{array}$ & $\begin{array}{l}\text { Dynamic Anton Paar MCR } 102 \text { rheometer. Materials } \\
\text { analyzed in molten state; } \mathrm{T}=180^{\circ} \mathrm{C} \text {; Parallel plates } \\
\text { geometry, } 25-\mathrm{mm} \text { diameter; } \mathrm{G}^{\prime} \text { and } \mathrm{G}^{\prime \prime} \text { moduli were } \\
\text { registered; } 100 \text { to } 10^{-3} \mathrm{rad} / \mathrm{s} \text {. }\end{array}$ & & \\
\hline Cellulose nanofibrils & $\begin{array}{l}\text { Nitroxide TEMPO insertion } \\
\text { and NMP of HEMA }\end{array}$ & $\begin{array}{l}\text { Stress-strain } \\
\text { compression }\end{array}$ & $\begin{array}{l}\text { Apparatus: Instron } 5848 ; 50 \mathrm{~N} \text { load cell. Test: cyclic } \\
\text { compressive stress and strain; } 5 \mathrm{~mm} / \mathrm{min} \text { for } 70 \% \text { strain, } \\
\text { followed by the same releasing rate until zero loading, for } \\
2 \mathrm{~min} \text {. }\end{array}$ & & [124] \\
\hline Lignin & $\begin{array}{l}\text { Insertion of ACX onto lignin } \\
\text { to produce a RAFT } \\
\text { macrocontroller to } \\
\text { polymerize AM or AA. }\end{array}$ & $\begin{array}{l}\text { Viscosity determination } \\
\text { of an aqueous solution } \\
\text { of grafted material. }\end{array}$ & $\begin{array}{l}\text { Apparatus: Brookfield cone-and-plate viscometer. Steady } \\
\text { shear measurements using } 100 \mathrm{rpm} \text {. }\end{array}$ & & [139] \\
\hline
\end{tabular}


Table 11. Summary of chromatographic characterization techniques used for grafted materials.

\begin{tabular}{|c|c|c|c|c|c|}
\hline Backbone & Grafts & Technique & Property Measured & Application & Refs. \\
\hline $\begin{array}{l}\text { Lignin-RAFT } \\
\text { macrocontroller }\end{array}$ & $\begin{array}{l}\text { Poly(soybean oil } \\
\text { methacrylate) derivatives. } \\
\text { PSBMA, PSBMAH, } \\
\text { PSBMAEO. }\end{array}$ & GPC & $\begin{array}{l}\text { Mn and MWD of different lignin-g-poly(soybean) } \\
\text { synthesized materials: Lignin-g-PSBMA } \\
(31.9 \mathrm{M} \mathrm{g} / \mathrm{mol} / 2.7) \text {; Lignin-g-PSBMAH }(57.1 \mathrm{M} \\
\mathrm{g} / \mathrm{mol} / 4.4) \text {; Lignin-g-PSBMAEO }(64.3 \mathrm{M} \mathrm{g} / \mathrm{mol} / 4.0) \text {. } \\
\mathrm{T}=35^{\circ} \mathrm{C} \text {; refractive index detector. Columns: HR1, HR3 } \\
\text { and HR5E. Solvent: THF at } 1 \mathrm{~mL} / \mathrm{min} \text {. Calibration with } \\
\text { polystyrene standards. Samples prepared in THF } \\
(4 \mathrm{mg} / \mathrm{mL}) \text { and filtered using a } 0.2 \mathrm{~mm} \text { mesh. }\end{array}$ & & [134] \\
\hline $\begin{array}{l}\text { Hydroxypropyl } \\
\text { cellulose (HPC) }\end{array}$ & $\begin{array}{l}\text { Steglich esterification of } \\
\text { PABTC over HPC using DCC } \\
\text { and DMAP, and grafting of } \\
\text { PABTC, with further } \\
\text { polymerization of EA and } \\
\text { NIPAAM }\end{array}$ & SEC & $\begin{array}{l}\text { MWD by SEC; two PL polar gel M and one PL polar gel } \\
5 \mathrm{~mm} \text { guard columns; refractive index detector; Eluent: } \\
\mathrm{N}, \mathrm{N} \text {-dimethylformamide/ } 0.3 \mathrm{M} \mathrm{LiBr}, 0.5 \mathrm{~mL} / \mathrm{min} \text {. }\end{array}$ & & [108] \\
\hline Cellulose acetate & $\begin{array}{l}\text { Solvents: } \mathrm{DMSO}, \mathrm{PDX} \text {, } \\
\text { DMAc, } \mathrm{C}_{3} \mathrm{H}_{6} \mathrm{O} \text {; initiators for } \\
\text { grafting and polymerization: } \\
\mathrm{CAN}, \mathrm{Sn}(\mathrm{Oct})_{2} \text { and BPO. }\end{array}$ & GPC & $\begin{array}{l}\text { Number average molecular weights }\left(\mathrm{M}_{\mathrm{n}}\right) \text { and dispersity } \\
\text { values }(Đ) \text { of grafted PMMA extracted from samples of } \\
\text { graft copolymer. Apparatus: Agilent } 1100 \text { with } 3 \text { PSS GPC } \\
8300 \mathrm{~mm}, 5 \mathrm{~mm}, 10^{6}, 10^{5}, 10^{3} \text { A columns; eluent: THF, } \\
0.8 \mathrm{~mL} / \mathrm{min} \text {, at } 20^{\circ} \mathrm{C} \text {. Calibration using polystyrene } \\
\text { standards with molecular weights ranging } 200-10^{6} \mathrm{~g} / \mathrm{mol} \text {. }\end{array}$ & & [111] \\
\hline $\begin{array}{l}\text { Microcrystalline } \\
\text { cellulose }\end{array}$ & $\begin{array}{l}\text { ROP of L-LA with DMAP in } \\
\text { an ionic liquid (AmimCl) to } \\
\text { produce Cellulose- } g \text {-PLLA. }\end{array}$ & HPLC & $\begin{array}{l}\text { Apparatus: Agilent } 1200 \text { with an XDB-C18 phase column. } \\
\text { Eluent: } \mathrm{H}_{2} \mathrm{O}-\text { methanol ( } 20: 80 \text { by vol.), } 0.8 \mathrm{~mL} / \mathrm{min} \text {. }\end{array}$ & & [116] \\
\hline
\end{tabular}


Table 11. Cont.

\begin{tabular}{|c|c|c|c|}
\hline Backbone & Grafts & Technique & Property Measured \\
\hline Hemicellulose & $\begin{array}{l}\text { Hemicellulose grafting using } \\
\text { TBD and e-caprolactone } \\
\text { monomers. }\end{array}$ & GPC & $\begin{array}{l}\text { Apparatus: Malvern Viscotek HT GPC } 350 \text {; refractive index } \\
\text { (RI) and viscometer detectors. PSS-GRAM columns } \\
\text { covering a range of } 100-1,000,000 \mathrm{~g} / \text { mol. Eluent: DMSO at } \\
80^{\circ} \mathrm{C} \text {. Universal calibration using pullulan polysaccharide } \\
\text { standards. }\end{array}$ \\
\hline Lignin & $\begin{array}{l}\text { Insertion of ACX onto lignin } \\
\text { to produce a RAFT } \\
\text { macro-controller which } \\
\text { polymerizes AM and AA. }\end{array}$ & GPC & $\begin{array}{l}\text { Apparatus: Waters Alliance } 2695 \text {; eluent: aqueous solution } \\
\text { of } 0.1 \mathrm{M} \text { sodium phosphate buffer and } 0.01 \% \mathrm{NaN}_{3} \text {, at } \\
\text { ambient temperature, } 1 \mathrm{~mL} / \mathrm{min} \text {. }\end{array}$ \\
\hline
\end{tabular}


Table 12. Summary of characterization techniques based on mechanical properties used for grafted materials.

\begin{tabular}{|c|c|c|c|c|c|}
\hline Backbone & Grafts & Technique & Property Measured & Application & Refs. \\
\hline $\begin{array}{l}\text { Lignin-RAFT } \\
\text { macro-controller }\end{array}$ & $\begin{array}{l}\text { Poly(soybean oil } \\
\text { methacrylate) derivatives. } \\
\text { PSBMA, PSBMAH, } \\
\text { PSBMAEO. }\end{array}$ & Tensile strength & $\begin{array}{l}\text { TT s/e values for Lignin-g-PSBMAH }(1.5 \mathrm{MPa} / 220 \%) \text {, } \\
\text { PSBMAH ( } 3.0 \mathrm{MPa} / 120 \%) \text { and epoxy resin prepared from } \\
\text { grafted materials }(17 \mathrm{MPa} / 22.5 \%) \text {. Tensile tests were } \\
\text { carried out using a crosshead speed of } 20 \mathrm{~mm} / \mathrm{min} \text { at } \\
\text { ambient temperature. Samples were casted to obtain films. } \\
\text { THF polymer solution samples were dried for } 12 \mathrm{~h} \text { under } \\
\text { vacuum at ambient temperature and for } 6 \mathrm{~h} \text {, at } 60^{\circ} \mathrm{C} \text {. Films } \\
\text { were cut into dog-bone tensile samples of } 20 \mathrm{~mm} \text { length } \\
\text { and } 5 \mathrm{~mm} \text { width. }\end{array}$ & & [134] \\
\hline $\begin{array}{l}\text { Cellulosic Grewia optiva } \\
\text { fibers }\end{array}$ & $\begin{array}{l}\text { FAS- } \mathrm{H}_{2} \mathrm{O}_{2} \text { redox initiation for } \\
\text { grafting of MA and KPS, } \\
\text { followed by polymerization } \\
\text { of MA. }\end{array}$ & Swell index & $\begin{array}{l}\text { Swelling of raw and grafted fibers in different solvents } \\
\text { (DMF, water, methanol, and isobutyl alcohol) was } \\
\text { measured. }\end{array}$ & & [110] \\
\hline $\begin{array}{l}\text { Cellulose nanocrystal } \\
\text { (CNC) }\end{array}$ & $\begin{array}{l}\text { Macromolecular initiator } \\
\text { obtained from the reaction } \\
\text { between Br-iBuBr and CNC } \\
\text { using TEA as catalyst, via } \\
\text { SI-ATRP with Sty. Material } \\
\text { was casted in PMMA. }\end{array}$ & Tensile strength & $\begin{array}{l}\text { The mechanical properties of nanocomposites were } \\
\text { investigated through breaking strength and elongation at } \\
\text { break tests. }\end{array}$ & & [118] \\
\hline $\begin{array}{l}\text { Cellulose cotton fiber } \\
\text { pulp }\end{array}$ & $\begin{array}{l}\text { Esterification of maleic } \\
\text { anhydride grafted over PHA } \\
\text { (through double bonds). } \\
\text { PHA-g-MA grafted onto } \\
\text { cellulose cotton fiber pulp } \\
\text { (through the anhydride } \\
\text { group). }\end{array}$ & Tensile strength & $\begin{array}{l}\text { Apparatus: Lloyd Instruments, Model LR5 } \mathrm{K} ; 100 \mathrm{~mm} / \mathrm{min} \\
\text { (ASTM D638); pieces sized } 150 \times 15 \mathrm{~mm} \text { (length } \times \text { width). } \\
\text { Mean values were determined from measurements from } \\
\text { four specimens. }\end{array}$ & & [120] \\
\hline
\end{tabular}


Table 12. Cont.

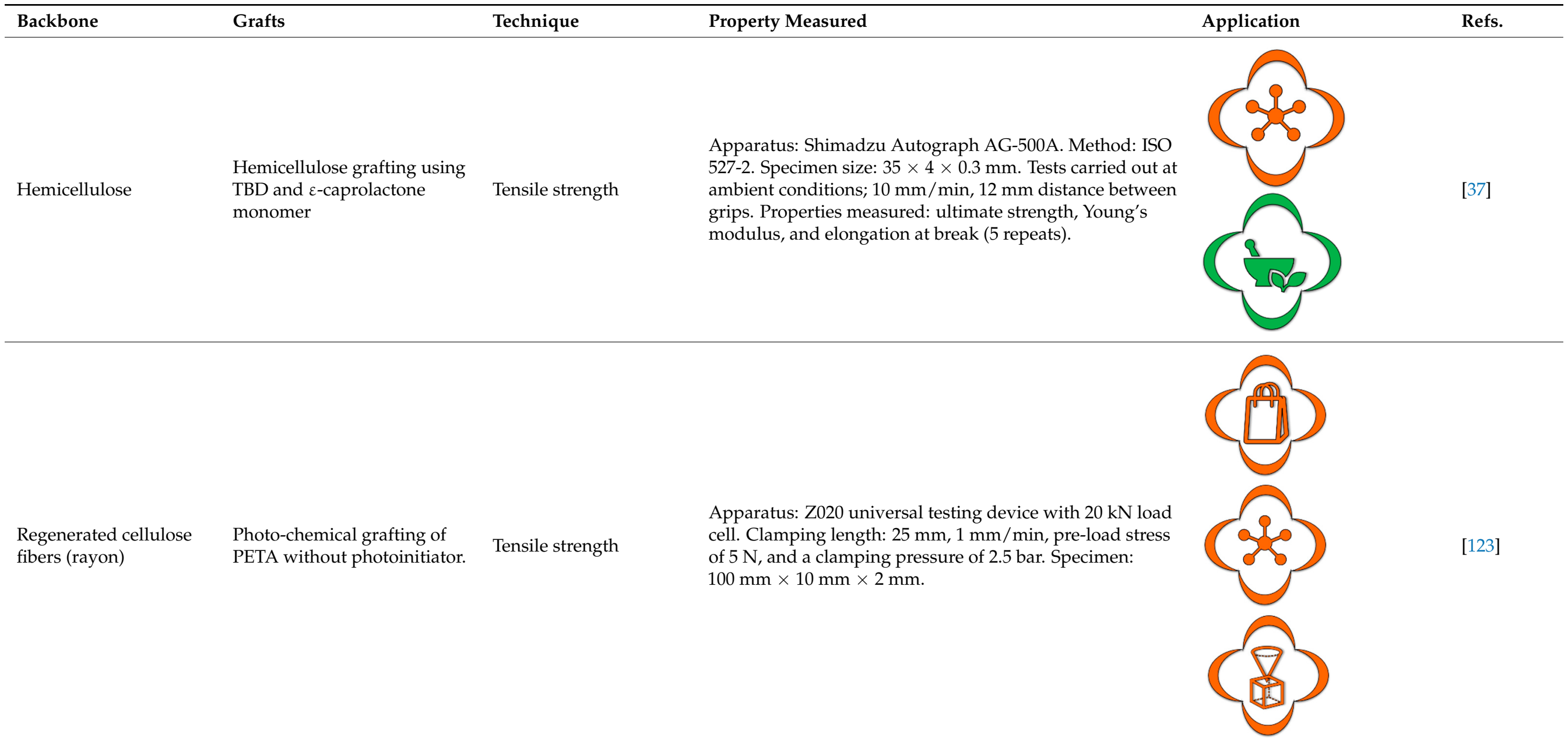

Apparatus: 858 Mini Bionix II, MTS testing machine,

Systems Corporation. Cyclic tests under tensile stresses

Fatigue

$(\mathrm{R}=0.1)$ were performed. Experiments reaching total cycle

numbers of 1 million were considered as runouts. Fatigue

tests performed uniformly at $10 \mathrm{~Hz}$. Experiments

proceeded until breakage or reaching runout conditions. 
Table 13. Summary of biological, functional, and compositional characterization techniques employed in grafted materials.

\begin{tabular}{|c|c|c|c|c|c|}
\hline Backbone & Grafts & Technique & Property Measured & Application & Refs. \\
\hline CellClAc & $\begin{array}{l}\text { Macro initiator, } \\
\mathrm{Cu}(\mathrm{I}) \mathrm{Cl} / 2^{\prime} 2^{\prime} \mathrm{BIPI} \text { catalytic } \\
\text { system via ATRP of } 4 \text { NPA and } \\
\text { MMA }\end{array}$ & Elemental analysis & $\begin{array}{l}\text { Apparatus: Leco CHNS-932. Elements determined: C, N } \\
\text { and H elements. }\end{array}$ & & [109] \\
\hline CellClAc & $\begin{array}{l}\text { Macro initiator, } \\
\mathrm{Cu}(\mathrm{I}) \mathrm{Cl} / 2^{\prime} 2^{\prime} \mathrm{BIPI} \text { catalytic } \\
\text { system via ATRP controller. }\end{array}$ & Elemental analysis & $\begin{array}{l}\text { Apparatus: Leco CHNS-932. Elements determined: C, N } \\
\text { and H elements. }\end{array}$ & & [112] \\
\hline Cellulose cotton fibers & $\begin{array}{l}\mathrm{Na}_{2} \mathrm{CO}_{3} \text { and thermal activation } \\
\text { using MTC-b-CD and silver } \\
\text { nitrate }\end{array}$ & $\begin{array}{l}\text { Microbiological } \\
\text { investigations }\end{array}$ & $\begin{array}{l}\text { Materials treated with Ag0 and Ag+ were evaluated for } \\
\text { antibacterial activity. Gram-positive coccus } \\
\text { (Staphylococcus aureus ATCC 25923) and Gram-negative } \\
\text { bacillus (Escherichia coli ATCC 25922) microorganisms } \\
\text { were used. The Kirby-Bauer diffusimetrical method was } \\
\text { used to achieve this purpose. Bacterial cultures were } \\
\text { standardized according to McFarland scale for } 18 \mathrm{~h} \text {, } \\
\text { yielding } 10^{7}-10^{8} \mathrm{CFU} / \mathrm{mL} \text {. After inoculation, sample } \\
\text { discs were placed on the surface of the medium. } \\
\text { Antibacterial activity was assessed after } 24 \mathrm{~h} \text { of } \\
\text { incubation, at } 37^{\circ} \mathrm{C} \text {. }\end{array}$ & & [113] \\
\hline $\begin{array}{l}\text { Microcrystalline } \\
\text { cellulose }\end{array}$ & $\begin{array}{l}\text { ROP of L-LA with DMAP in an } \\
\text { ionic liquid (AmimCl) to } \\
\text { produce Cellulose- } g \text {-PLLA }\end{array}$ & $\begin{array}{l}\text { Load and controlled } \\
\text { release }\end{array}$ & $\begin{array}{l}\text { A solution of grafted material }(60.0 \mathrm{mg}) \text { and vitamin C } \\
(60.0 \mathrm{mg}) \text { in } 2 \mathrm{~mL} \text { of PBS was prepared and then } \\
\text { transferred to a dialysis bag. Dialysis against } 1 \mathrm{~L} \text { distilled } \\
\text { water for } 24 \mathrm{~h} \text { (water refreshment after } 12 \mathrm{~h} \text { ) followed. } \\
\text { Afterwards, the dialysis bag was immersed into a } 400 \mathrm{~mL} \\
\text { phosphate buffer solution, at conditions similar to those } \\
\text { of intestinal fluid (pH 7.40) at } 37^{\circ} \mathrm{C} .2 \mathrm{~mL} \text { samples were } \\
\text { taken at predetermined times, and analyzed at } 1_{\max }= \\
245 \mathrm{~nm} \text { for vitamin C. }\end{array}$ & & [116] \\
\hline
\end{tabular}


Table 13. Cont.

\begin{tabular}{|c|c|c|c|c|c|}
\hline Backbone & Grafts & Technique & Property Measured & Application & Refs. \\
\hline \multirow[b]{2}{*}{$\begin{array}{l}\text { Cellulose cotton fiber } \\
\text { pulp }\end{array}$} & \multirow{2}{*}{$\begin{array}{l}\text { Esterification of maleic } \\
\text { anhydride grafted onto PHA } \\
\text { (through double bonds). } \\
\text { PHA-g-MA grafted onto } \\
\text { cellulose cotton fiber pulp } \\
\text { (through the anhydride group). }\end{array}$} & Surface roughness & $\begin{array}{l}\text { Measurement of surface roughness accomplished using } \\
\text { an L\&M CE165 PPS tester. }\end{array}$ & & \multirow[b]{2}{*}{ [120] } \\
\hline & & $\begin{array}{l}\text { Contact angle } \\
\text { (hydrophobicity) }\end{array}$ & $\begin{array}{l}\text { Surface hydrophilicity was analyzed by contact angle } \\
\text { measurements. Tests carried out at ambient conditions } \\
\text { using a Dataphysics OCA } 40 \text { Micro instrument. Surface } \\
\text { free energy parameters were estimated from these data. }\end{array}$ & & \\
\hline Cellulosic filter papers & $\begin{array}{l}\text { Radiation-induced graft } \\
\text { (RIGCP) copolymerization of } \\
\text { acrylonitrile. }\end{array}$ & Chelating rare elements & $\begin{array}{l}\text { Chelation of uranium, thorium, and lanthanides by the } \\
\text { batch procedure took place. }\end{array}$ & & [121] \\
\hline \multirow{3}{*}{ Hemicellulose } & \multirow{3}{*}{$\begin{array}{l}\text { Hemicellulose grafting using } \\
\text { TBD and } \varepsilon \text {-caprolactone } \\
\text { monomers. }\end{array}$} & Contact angle & $\begin{array}{l}\text { Measurements carried out using an NRL Contact Angle } \\
\text { Goniometer by Rame-Hart, model 100-00. }\end{array}$ & & \multirow{3}{*}{ [37] } \\
\hline & & & & & \\
\hline & & Biodegradation test & $\begin{array}{l}\text { Method: ISO 14851. Biochemical Oxygen Demand (BOD) } \\
\text { measurements were obtained under aerobic conditions. }\end{array}$ & & \\
\hline
\end{tabular}


Table 13. Cont.

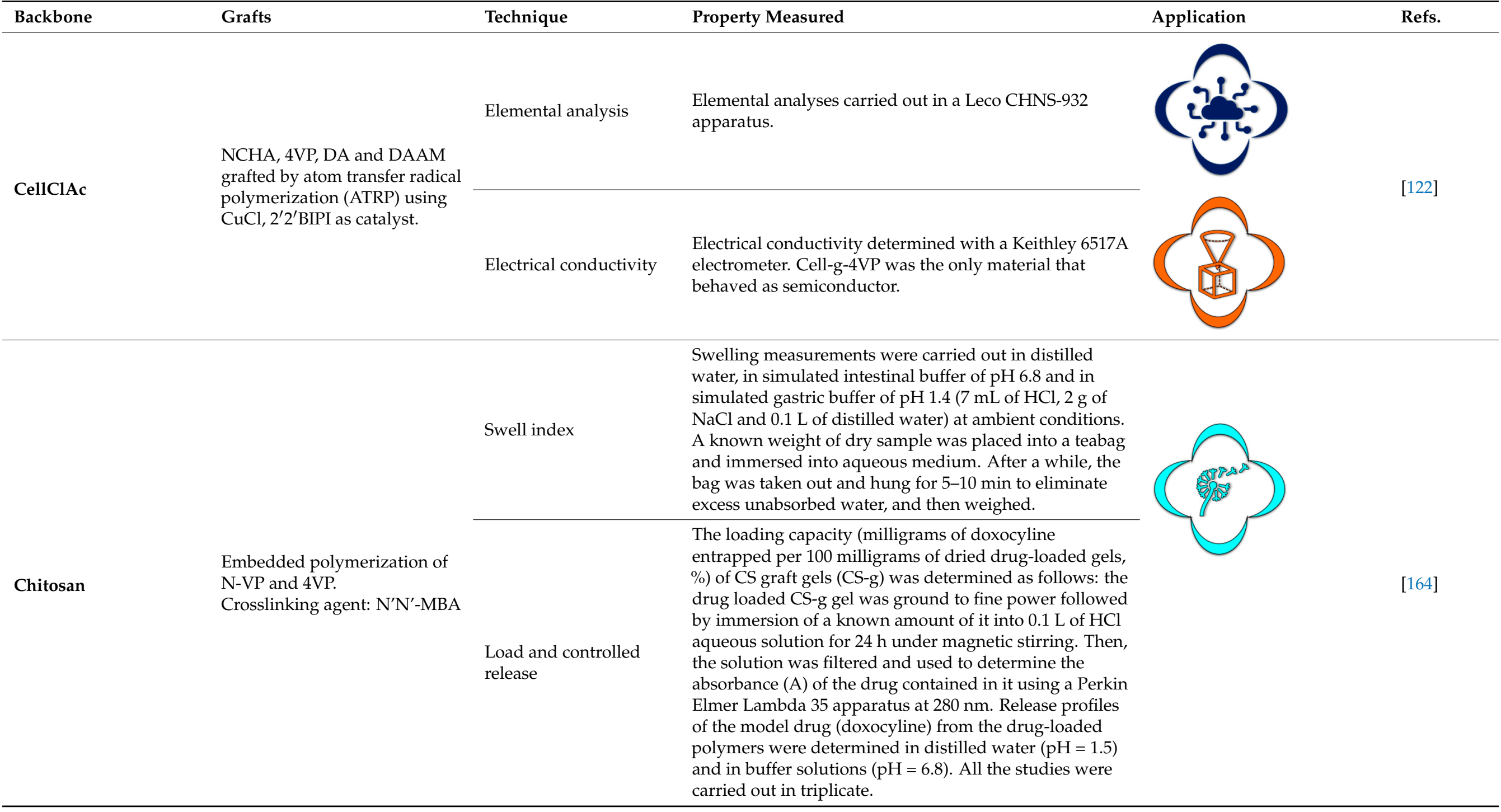


Table 13. Cont.

Regenerated cellulose fibres (rayon)

Photo-chemical grafting of PETA without photoinitiator.

Volume of materials was determined by measuring the dimensions of samples (calliper gauge 500-171-20,

Mitutoyo), and from grammage and density of fibers, referred to the total mass determined using a type 440 $35 \mathrm{~N}$ fine-scale.

Apparatus: NOVA 1200e Quantachrome. Method: Brunauer-Emmett-Teller (BET) method for analysis of $\mathrm{N}_{2}$ gas adsorption isotherms. Estimated properties: specific surface area (SBET) and average pore volumes. BET surface area improved from $9.87 \mathrm{~m}^{2} / \mathrm{g}$ for

TEMPO-Cellulose to $19.7283 \mathrm{~m}^{2} / \mathrm{g}$ for

BET

HEMA-TEMPO-Cellulose. The microporous volume

(Vtotal) increased from $0.0484 \mathrm{~cm}^{3} / \mathrm{g}$ to $0.0705 \mathrm{~cm}^{3} / \mathrm{g}$, for HEMA-TEMPO-Cellulose.

and NMP of HEMA

Electrical resistivity determined using a four-point probe system (ST 2253, Suzhou Jingge Electronic Co.). The direct freezing method was compared against the unidirectional gradient freezing method. Samples produced using the direct freezing method exhibited higher resistivity values.

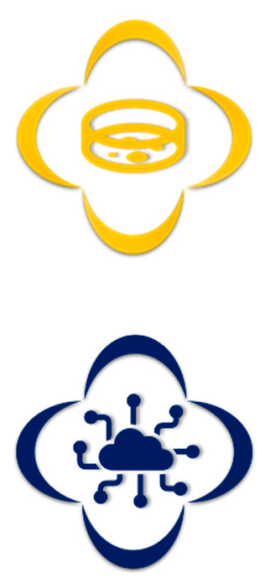


Table 13. Cont.

\begin{tabular}{|c|c|c|c|c|c|}
\hline Backbone & Grafts & Technique & Property Measured & Application & Refs. \\
\hline & & Surface tension & $\begin{array}{l}\text { Apparatus: De Nouy ring-type tensiometer (Krüss), at } 25 \\
{ }^{\circ} \mathrm{C} \text {. }\end{array}$ & & \\
\hline Lignin & $\begin{array}{l}\text { Insertion of ACX onto lignin to } \\
\text { produce a RAFT } \\
\text { macro-controller that } \\
\text { polymerizes AM and AA }\end{array}$ & Emulsification test & $\begin{array}{l}\text { Absorbance of solutions of dissolved grafted material in } \\
\text { deionized water and mixtures of hexanes (ultrasonication } \\
\text { required for disolution) was measured using a Cary } 300 \\
\text { spectrophotometer. Comparison of this value with that of } \\
\text { the starting aqueous solution allowed calculation of } \\
\text { lignin content in the emulsion phase. }\end{array}$ & & [139] \\
\hline
\end{tabular}


Table 14. Overview of studies focused on the modeling of polymer grafting.

\begin{tabular}{|c|c|c|c|c|c|}
\hline Backbone & Functionalization Method & Graft Chains & Modeling Approach & Highlights/Comments & Ref. \\
\hline $\begin{array}{l}\text { Pre-polymer containing highly } \\
\text { active chain transfer sites } \\
\text { (pendant mercaptan groups). }\end{array}$ & $\begin{array}{l}\text { CTP between polymer } \\
\text { radicals and pendant } \\
\text { mercaptan groups. }\end{array}$ & Vinyl polymer & $\begin{array}{l}\text { Kinetic model for FRP including CTP; } \\
\text { model equations solved numerically } \\
\text { and approximate closed form } \\
\text { equations were also used. }\end{array}$ & $\begin{array}{l}\text { Calculated properties/variables: } \\
\text { grafting efficiency (Equation (1)) and } \\
\text { number average molecular weights of } \\
\text { the different polymer molecules } \\
\text { (Equations (2)-(6)). }\end{array}$ & [39] \\
\hline Solid polymeric substrates. & $\begin{array}{l}\text { Irradiation of } \\
\text { polymer/monomer } \\
\text { mixture. }\end{array}$ & Vinyl polymer & $\begin{array}{l}\text { Calculation of } r_{n}, r_{w} \text { and number full } \\
\text { chain length distributions }\left(n_{x}\right) \text { from } \\
\text { kinetic equations. }\end{array}$ & $\begin{array}{l}\text { Two limiting cases were considered in } \\
\text { the calculation of } n_{\mathrm{x}} \text { : (a) radicals } \\
\text { terminating by disproportion, no } \\
\text { significant chain transfer and complete } \\
\text { conversion; and (b) no chain transfer } \\
\text { and incomplete conversion (see } \\
\text { Equation (7)). }\end{array}$ & [40] \\
\hline Polyolefins & High temperature CTP. & Vinyl polymer & $\begin{array}{l}\text { Kinetic modeling from a given } \\
\text { polymerization scheme. }\end{array}$ & $\begin{array}{l}\text { Review paper considering chemical } \\
\text { modification of polyolefins by FRP in } \\
\text { extruders. }\end{array}$ & [231] \\
\hline Silica substrate & $\begin{array}{l}\text { Formation of a growing } \\
\text { grafted polymer chain from } \\
\text { reaction between an } \\
\text { initiator derived primary } \\
\text { free radical and a "surface } \\
\text { site." }\end{array}$ & Poly(vinylpyrrolidone) & $\begin{array}{l}\text { Use of a "conservational } \\
\text { polymerization and molecular-weight } \\
\text { distribution" (CPMWD) numerical } \\
\text { method to solve the kinetic equations. }\end{array}$ & $\begin{array}{l}\text { The proposed numerical approach } \\
\text { provides monomer conversion, } \\
\text { grafting yield, and full MWDs of active } \\
\text { and inactive homopolymer and graft } \\
\text { polymer species. }\end{array}$ & [232] \\
\hline Porous silica beads & $\begin{array}{l}\text { Chain transfer reaction to } \\
\text { fixed mercapto groups } \\
\text { present in the } \\
\text { pre-functionalized silica } \\
\text { beads. }\end{array}$ & Polystyrene & $\begin{array}{l}\text { Kinetic model that allows calculation } \\
\text { of full MWD and Đ; no details on } \\
\text { numerical approach or software used } \\
\text { were provided. }\end{array}$ & $\begin{array}{l}\text { MWDs were approximated assuming } \\
\text { them, as a first approach, to follow a } \\
\text { normal distribution function. }\end{array}$ & [233] \\
\hline PE & $\begin{array}{l}\text { CTP by FRP in melt, in a } \\
\text { twin-screw extruder (TSE). }\end{array}$ & $\begin{array}{l}\text { Poly(glycidyl methacrylate) } \\
\text { (PGMA) }\end{array}$ & $\begin{array}{l}\text { Coupling of flow characteristics from a } \\
\text { TSE simulation program and a reactor } \\
\text { model consisting of plug flow and } \\
\text { "axial dispersion" reactor cells. }\end{array}$ & $\begin{array}{l}\text { Overall GMA conversion was } \\
\text { predicted, showing general good } \\
\text { agreement with experimental data. }\end{array}$ & [234] \\
\hline PE & $\begin{array}{l}\text { CTP by FRP in melt, in a } \\
\text { twin-screw extruder (TSE). }\end{array}$ & $\begin{array}{l}\text { Poly(maleic anhydride) } \\
\text { (PMAH) }\end{array}$ & $\begin{array}{l}\text { Coupling of reaction kinetics to flow } \\
\text { equations in a twin-screw extruder. }\end{array}$ & $\begin{array}{l}\text { Satisfactory agreement between } \\
\text { calculated and experimental data. }\end{array}$ & [235] \\
\hline
\end{tabular}


Table 14. Cont.

\begin{tabular}{|c|c|c|c|c|c|}
\hline Backbone & Functionalization Method & Graft Chains & Modeling Approach & Highlights/Comments & Ref. \\
\hline Polypropylene (PP) & CTP by FRP in melt & PMAH & $\begin{array}{l}\text { A kinetic model which captures the } \\
\text { effect of MAH and initiator } \\
\text { concentrations on grafting yield and } \\
\text { MWDs during the grafting process } \\
\text { was derived. }\end{array}$ & $\begin{array}{l}\text { Model performance assessed by } \\
\text { comparing calculated and } \\
\text { experimental data from different } \\
\text { sources, for batch (internal mixer, static } \\
\text { film) and continuous systems } \\
\text { (twin-screw extruder), under a wide } \\
\text { window of operating conditions. }\end{array}$ & [236] \\
\hline $\begin{array}{l}\text { Cellulose or cellulose } \\
\text { containing fibers }\end{array}$ & $\begin{array}{l}\text { Not specified (empirical } \\
\text { approach) }\end{array}$ & $\begin{array}{l}\text { Monochlorotriazinyl- } \\
\beta \text {-cyclodextrin }\end{array}$ & $\begin{array}{l}\text { Neural network modeling } \\
\text { (semi-empirical approach). }\end{array}$ & $\begin{array}{l}\text { A good approximation of the studied } \\
\text { grafting process was obtained. }\end{array}$ & [237-239] \\
\hline PP & $\begin{array}{l}\text { CTP by FRP in solid state, } \\
\text { in presence and absence of } \\
\text { supercritical } \mathrm{CO}_{2}\left(\mathrm{scCO}_{2}\right) \text {. }\end{array}$ & PMAH & $\begin{array}{l}\text { A kinetic model focused on } \\
\text { polymerization and grafting rates was } \\
\text { used. }\end{array}$ & $\begin{array}{l}\text { The effect of presence or absence of } \\
\mathrm{scCO}_{2} \text { on grafting rate was analyzed. }\end{array}$ & [240] \\
\hline PP & $\begin{array}{l}\text { CTP by FRP in solid state, } \\
\text { with and without } \mathrm{scCO}_{2} \text {. }\end{array}$ & Not specified & $\begin{array}{l}\text { The kinetic model and grafting } \\
\text { mechanism of the } \mathrm{scCO}_{2} \text { assisted } \\
\text { grafting of PP in the solid state were } \\
\text { reviewed. }\end{array}$ & $\begin{array}{l}\text { Grafting rate controlled by diffusion in } \\
\text { absence of } \mathrm{scCO}_{2} \text { and by the chemical } \\
\text { grafting step in presence of } \mathrm{scCO}_{2} \text {. }\end{array}$ & [241] \\
\hline Polybutadiene (PB) & $\begin{array}{l}\text { CTP by FRP in production } \\
\text { of high-impact PSty (HIPS). }\end{array}$ & PSty & $\begin{array}{l}\text { An existent heterogeneous model is } \\
\text { generalized to consider bivariate } \\
\text { distributions of graft copolymer } \\
\text { topologies; each topology is } \\
\text { determined by the number of } \\
\text { trifunctional branching points per } \\
\text { molecule. }\end{array}$ & $\begin{array}{l}\text { Polymerization in two phases is } \\
\text { assumed. Adjustment of a single } \\
\text { kinetic parameter is required. Grafting } \\
\text { efficiencies obtained from solvent } \\
\text { extraction/gravimetry are contrasted } \\
\text { against estimates obtained by the } \\
\text { deconvolution of size exclusion } \\
\text { chromatograms for total polymer. }\end{array}$ & [242] \\
\hline Poly[ethylene-co-(1- octene)] & CTP by FRP in molten state & PMMA & $\begin{array}{l}\text { A model compound approach was } \\
\text { used. }\end{array}$ & $\begin{array}{l}\text { The focus was on assessing the effect } \\
\text { of temperature, polymerization time } \\
\text { and initiator content on MMA } \\
\text { polymerization rate and number } \\
\text { average chain length, in the presence } \\
\text { of alkoxyl radicals and alkanes. }\end{array}$ & [243] \\
\hline
\end{tabular}


Table 14. Cont.

\begin{tabular}{|c|c|c|c|c|c|}
\hline Backbone & Functionalization Method & Graft Chains & Modeling Approach & Highlights/Comments & Ref. \\
\hline PSty & $\begin{array}{l}\text { Use of a Friedel-Crafts } \\
\text { reaction, which gives rise to } \\
\text { a mixture of unmodified PE, } \\
\text { modified PS and a PE-g-PS } \\
\text { copolymer. }\end{array}$ & $\mathrm{PE}$ & $\begin{array}{l}\text { A model for predicting the complete } \\
\text { MWD of the homopolymers, and the } \\
\text { chemical composition distribution } \\
\text { (CCD) of the graft copolymer was } \\
\text { presented. Probability generating } \\
\text { functions were used to carry out the } \\
\text { calculations. }\end{array}$ & $\begin{array}{l}\text { The model takes into account the } \\
\text { opposing effects of rival reactions that } \\
\text { occur in the studied grafting reaction. }\end{array}$ & {$[244,245]$} \\
\hline PE & CTP by FRP & PSty & $\begin{array}{l}\text { A kinetic mechanism for the whole } \\
\text { process and application of the method } \\
\text { of moments to solve the mass balance } \\
\text { equations are proposed. }\end{array}$ & $\begin{array}{l}\text { The model is able to calculate average } \\
\text { molecular weights and the amount of } \\
\text { grafted PS. }\end{array}$ & [246] \\
\hline PP & CTP by FRP & PMAH & $\begin{array}{l}\text { A kinetic model for the free-radical } \\
\text { grafting of maleic anhydride MAH } \\
\text { onto PP, considering that the reaction } \\
\text { medium is heterogeneous due to the } \\
\text { incomplete solubility of MAH in the } \\
\text { polymer melt was developed. }\end{array}$ & $\begin{array}{l}\text { Two different approaches are } \\
\text { considered that derive from the } \\
\text { limiting cases of very fast mass } \\
\text { transfer (equilibrium) and no mass } \\
\text { transfer between the two phases. The } \\
\text { physical parameters considered in the } \\
\text { model include the solubility of MAH } \\
\text { in PP and the partition coefficient of } \\
\text { the initiator between the two phases. }\end{array}$ & [247] \\
\hline $\begin{array}{l}\text { Carboxyl functionalized } \\
\text { hydrophilic Sephadex (a } \\
\text { cross-linked dextran gel used } \\
\text { for gel filtration) derivatives. }\end{array}$ & $\begin{array}{l}\text { Covalent amine } \\
\text { conjugation method } \\
\text { analyzed using XPS. }\end{array}$ & Poly(ethylene glycol) (PEG) & $\begin{array}{l}\text { Langmuir and Langmuir-Freundlich } \\
\text { isotherm models are used to study } \\
\text { PEG-grafting kinetics. Fractional C-O } \\
\text { intensities obtained from } \\
\text { high-resolution } C 1 \text { s scans are } \\
\text { correlated with grafting. }\end{array}$ & $\begin{array}{l}\text { The models are assessed by comparing } \\
\text { calculated profiles and experimental } \\
\text { data. }\end{array}$ & [248] \\
\hline
\end{tabular}


Table 14. Cont.

\begin{tabular}{|c|c|c|c|c|c|}
\hline Backbone & Functionalization Method & Graft Chains & Modeling Approach & Highlights/Comments & Ref. \\
\hline Various surfaces & $\begin{array}{l}\text { Surface-initiated controlled } \\
\text { radical polymerization. }\end{array}$ & $\begin{array}{l}\text { Polymers from } \\
\text { 2-methacryloyloxythyl } \\
\text { phosphorylcholine (MPC), } \\
\text { methyl acrylate, acrylamide, } \\
\text { and N-isopropylacrylamide. }\end{array}$ & $\begin{array}{l}\text { A kinetic model for surface-initiated } \\
\text { atom transfer radical polymerization } \\
\text { (SI-ATRP) with addition of excess } \\
\text { deactivator in solution was presented. }\end{array}$ & $\begin{array}{l}\text { Polymer layer thickness, and } \\
\text { concentrations of radical, dormant and } \\
\text { dead polymer molecules can be } \\
\text { calculated. A simple but reliable } \\
\text { analytical solution is proposed for } \\
\text { estimation of the evolution of polymer } \\
\text { layer thickness. }\end{array}$ & [249] \\
\hline $\begin{array}{l}\text { Partially fluorinated polymers } \\
\text { such as poly(vinylidene } \\
\text { fluoride) and } \\
\text { poly(ethylene-co-tetrafluoro } \\
\text { ethene). }\end{array}$ & Radiation induced grafting & Poly(4-vinylpyridine) & $\begin{array}{l}\text { Box-Behnken factorial designs and } \\
\text { response surface method (RSM) were } \\
\text { used. }\end{array}$ & $\begin{array}{l}\text { A quadratic model was used to obtain } \\
\text { the optimal set of conditions (absorbed } \\
\text { dose, monomer concentration, grafting } \\
\text { time and reaction temperature) to } \\
\text { maximize the degree of grafting (G\%), } \\
\text { using a statistical software. Adequate } \\
\text { agreement between model predictions } \\
\text { and experimental data supported the } \\
\text { approach proposed. }\end{array}$ & {$[250,251]$} \\
\hline Poly(propylene glycol) (PPG) & CTP by FRP & $\begin{array}{l}\text { Poly(styrene-co-acrylonitrile) } \\
\text { (SAN) }\end{array}$ & $\begin{array}{l}\text { A batch kinetic model for grafting } \\
\text { copolymerization of Sty and } \\
\text { acrylonitrile (AN) in the presence of } \\
\text { PPG is modified to include } \\
\text { semi-continuous and continuous } \\
\text { processes. }\end{array}$ & $\begin{array}{l}\text { The semi-continuous model is } \\
\text { validated using experimental data, } \\
\text { although molecular weight agreement } \\
\text { is poor. The continuous process is } \\
\text { simulated but not contrasted against } \\
\text { experimental data. According to the } \\
\text { model, graft efficiency increases } \\
\text { drastically, and Mw decreases with } \\
\text { decreasing St/AN weight ratio. }\end{array}$ & [252] \\
\hline Starch & $\begin{array}{l}\text { (Mechanically activated) } \\
\text { CTP by FRP }\end{array}$ & Polyacrylamide & $\begin{array}{l}\text { A kinetic model for an inverse } \\
\text { emulsion copolymerization system } \\
\text { was used. The effects of initiator, } \\
\text { monomer, starch, and emulsifier } \\
\text { content on reaction rate of graft } \\
\text { co-polymerization (Rg) were } \\
\text { determined. The kinetic model was } \\
\text { modified to provide acceptable } \\
\text { agreement with experimental data. }\end{array}$ & $\begin{array}{l}\text { It was observed that the relationship } \\
\text { between } \mathrm{Rg} \text { and the concentration of } \\
\text { components in the polymerization } \\
\text { system can be expressed for all of the } \\
\text { four components by the following } \\
\text { equation: } \operatorname{Rg} \infty[\mathrm{mSt}] 1.24 \\
{[\mathrm{I}] 0.76[\mathrm{M}] 1.54[\mathrm{E}] 0.33 \text {, which is }} \\
\text { consistent with the kinetic equation } \\
\text { from theoretical studies: } \\
\text { Rp } \infty[\mathrm{mSt}] 0.5 \sim 1[\mathrm{I}] 0.5 \sim 1[\mathrm{M}] 1 \sim 1.5[\mathrm{E}] 0.6 \text {. }\end{array}$ & [253] \\
\hline
\end{tabular}


Table 14. Cont.

\begin{tabular}{|c|c|c|c|c|c|}
\hline Backbone & Functionalization Method & Graft Chains & Modeling Approach & Highlights/Comments & Ref. \\
\hline PB seed latex particles & CTP by emulsion FRP & SAN & $\begin{array}{l}\text { Emulsion grafting copolymerization is } \\
\text { model using a two-phase kinetic } \\
\text { model. Monomer concentrations in the } \\
\text { different phases is calculated. }\end{array}$ & $\begin{array}{l}\text { Partition of components between } \\
\text { phases calculated as in previous } \\
\text { models. The model is extended to } \\
\text { account for calculation of grafting } \\
\text { efficiency and copolymer composition } \\
\text { of free and grafted molecules. }\end{array}$ & [254] \\
\hline PE & CTP by FRP in REX & PAA & $\begin{array}{l}\text { Monomer conversion to homo- and } \\
\text { grated-polymers are calculated } \\
\text { separately using an incremental theory. } \\
\text { The approach allows calculation of } \\
\text { degree of grafting, mass of } \\
\text { homopolymer and grafting efficiency. }\end{array}$ & $\begin{array}{l}\text { The focus was on analyzing the effect } \\
\text { of temperature and initial } \\
\text { concentrations of monomer and } \\
\text { initiator on degree of grafting. Both } \\
\text { grafting and amount of homopolymer } \\
\text { increase with temperature and } \\
\text { monomer concentration. }\end{array}$ & [255] \\
\hline $\begin{array}{l}\text { Natural rubber latex (core-shell } \\
\text { particles) }\end{array}$ & CTP by emulsion FRP & PMMA & $\begin{array}{l}\text { The model consists on rate expressions } \\
\text { of polymer chain formation. A } \\
\text { decrease of CHPO by TEPA and a } \\
\text { population event of radicals between } \\
\text { core/shell phases are considered in the } \\
\text { model. }\end{array}$ & $\begin{array}{l}\text { Grafting efficiency and polymer } \\
\text { composition for free and grafted } \\
\text { polymer populations can be calculated } \\
\text { in terms of initial conditions (monomer } \\
\text { and initiator content, and rubber } \\
\text { weight ratio and temperature). }\end{array}$ & [256] \\
\hline $\begin{array}{l}\text { Vinyl/divinyl copolymer } \\
\text { synthesized by RAFT } \\
\text { chemistry. }\end{array}$ & CTP by FRP & $\begin{array}{l}\text { Non-specified RAFT } \\
\text { synthesized graft }\end{array}$ & $\begin{array}{l}\text { A RAFT crosslinking model was used } \\
\text { to address the molecular imprinting } \\
\text { step. The size of grafted brushes was } \\
\text { estimated from MWD calculations for } \\
\text { a RAFT homo-polymerization. }\end{array}$ & $\begin{array}{l}\text { The model includes the two steps } \\
\text { involved in the production of MIP } \\
\text { responsive particles. Limitations and } \\
\text { ways to improve the model are } \\
\text { discussed. }\end{array}$ & [257] \\
\hline High-density PE (HDPE) & $\begin{array}{l}\text { Melt free-radical grafting } \\
\text { (semi-empirical approach) }\end{array}$ & GMA & $\begin{array}{l}\text { Response surface, desirability function, } \\
\text { and artificial intelligence approaches } \\
\text { were combined to account for polymer } \\
\text { grafting. Input variables: monomer } \\
\text { content, initiator concentration, and } \\
\text { melt-processing time. }\end{array}$ & $\begin{array}{l}\text { An in-house code that learns and } \\
\text { mimics processing torque and grafting } \\
\text { of GMA onto HDPE was developed. } \\
\text { Optimization of the process and } \\
\text { quantification of the competition } \\
\text { between grafting and crosslinking was } \\
\text { carried out. }\end{array}$ & [258] \\
\hline
\end{tabular}


Table 14. Cont.

\begin{tabular}{|c|c|c|c|}
\hline Backbone & Functionalization Method & Graft Chains & Modeling Approach \\
\hline PE & $\begin{array}{l}\text { CTP by FRP in } \\
\text { post-polymerization } \\
\text { modification }\end{array}$ & Vinyl polymers & $\begin{array}{l}\text { A kinetic Monte-Carlo (kMC) } \\
\text { modeling approach for free radical } \\
\text { induced grafting of vinyl monomers } \\
\text { onto polyethylene (PE), assuming } \\
\text { isothermicity, perfect macromixing and } \\
\text { diffusion-controlled effects was } \\
\text { developed. }\end{array}$ \\
\hline
\end{tabular}

CTP by FRP (batch

operation)

Vinyl polymers

A kMC model was used. Reactions included: initiator dissociation, hydrogen abstraction, graft chain initiation, graft (de)propagation, crosslinking, and homo (de) polymerization

\section{Highlights/Comments}

Ref

Calculated variables: monomer

conversion, grafting selectivity and yield, MWDs of all macromolecular

species involved, average grafting

(from/to) and crosslink densities, number of grafts and crosslinks per ndividual polymer molecule, and chain length of every graft.

Assumptions: isothermicity and single-phase polymerization. It is found that the grafting kinetics is affected by hydrogen abstraction and depropagation kinetic rate constants, equilibrium coefficient Keq, and initial content of monomer, initiator, and polyolefin.

They found that functionalization, selectivity and grafting density are able to reach remarkably better value (e.g., $100 \%$ ) if multiple injection points are considered for monomer, initiator and/or a temperature profile.

The partition of monomer and initiator between the two phases is addressed by introducing overall mass transfer coefficients. The model is tested with a monomer with low to negligible

homo-propagation. Positive agreement with industrial experimental data was obtained. 
Table 15. Polymerization scheme for free-radical polymerization including CTP and crosslinking.

\begin{tabular}{|c|c|c|}
\hline Reaction Step & Kinetic Expression & Remarks \\
\hline Initiator decomposition & $I \stackrel{k_{d}}{\rightarrow} 2 R$ & \\
\hline First propagation & $R+M \stackrel{k_{i}}{\rightarrow} P_{1}$ & \\
\hline Propagation & $P_{n}+M \stackrel{k_{p}}{\rightarrow} P_{n+1}$ & $\mathrm{n}=1, \ldots \infty$ \\
\hline Termination by disproportionation & $P_{n}+P_{m} \stackrel{k_{t d}}{\rightarrow} D_{n}+D_{m}$ & $\mathrm{n}, \mathrm{m}=1, \ldots \infty$ \\
\hline Propagation through intermediate free radicals & $P_{n, b}+M \stackrel{k_{p}}{\rightarrow} P_{n+1, b}$ & $\begin{array}{l}\mathrm{n}=1, \ldots \infty \\
\mathrm{b}=0, \ldots \infty\end{array}$ \\
\hline Chain transfer to polymer & $P_{n, b}+D_{m, c} \stackrel{k_{t r p}}{\rightarrow} D_{n, b}+P_{m, c+1}$ & $\begin{array}{l}\mathrm{n}, \mathrm{m}=1, \ldots \infty \\
\mathrm{b}, \mathrm{c}=0, \ldots \infty\end{array}$ \\
\hline $\begin{array}{l}\text { Propagation through pendant double bonds (crosslinking), } \\
\text { assuming a pseudo-kinetic rate constants method } \\
\text { (pseudo-homopolymer) approach }\end{array}$ & $P_{r}+D_{s} \stackrel{k_{p}^{* 0}}{\rightarrow} P_{r+s}$ & $\mathrm{r}, \mathrm{s}=1, \ldots \infty$ \\
\hline
\end{tabular}

Table 16. Symbols of application fields.

\begin{tabular}{|c|c|}
\hline Symbol of Corresponding Application & Meaning \\
\hline & Biocomposites and biomaterials. \\
\hline & Green chemistry and innovative processes. \\
\hline & Electronic materials, electrical properties, and conjugated polymers. \\
\hline & Surface modification, hydrophilic or hydrophobic surfaces. \\
\hline
\end{tabular}


Table 16. Cont.

\begin{tabular}{|c|c|}
\hline Symbol of Corresponding Application & Meaning \\
\hline & $\begin{array}{l}\text { Flame retardancy, additives for flame retardancy in polymer blends, thermal } \\
\text { resistance materials. }\end{array}$ \\
\hline & Antimicrobial applications. \\
\hline & Adhesives, polymer networks, crosslinked polymers, gels. \\
\hline & Controlled release of drugs and chemicals. \\
\hline & Polymer composites and blends, by extrusion, casting or co-precipitation. \\
\hline & Chelating properties, membranes, effluent remediation. \\
\hline
\end{tabular}


Table 16. Cont.

Symbol of Corresponding Application Meaning

Table 17. Abbreviations used for characterization techniques.

\begin{tabular}{ll}
\hline Abbreviation & Meaning \\
\hline FTIR & Fourier transformed mid-infrared spectroscopy. \\
\hline ATR & Attenuated total reflection. \\
\hline${ }^{1}$ H-NMR & Proton nuclear magnetic resonance. \\
\hline${ }^{13}$ C-NMR & Carbon nuclear magnetic resonance. \\
\hline TT & Tensile test. \\
\hline DMA & Dynamical mechanical analysis. \\
\hline GPC & Gel permeation chromatography. \\
\hline TGA & Thermogravimetric analysis. \\
\hline DSC & Differential scanning calorimetry. \\
\hline FR & Flame retardancy. \\
\hline XPS & X-ray photoelectron spectroscopy. \\
\hline EDAX & Energy-dispersive X-ray analysis/spectroscopy. \\
\hline WAXD & Wide Angle X-ray Scattering. \\
\hline XRF & X-ray fluorescence. \\
\hline
\end{tabular}


Table 18. Abbreviations of properties and variables measured by characterization techniques.

\begin{tabular}{|c|c|c|c|}
\hline Technique & Abbreviation & Explanation & Units \\
\hline \multirow{2}{*}{ Chromatography } & MWD & Molecular weight distribution of polymers. & - \\
\hline & $\mathrm{M}_{\mathrm{n}}$ & Number average molecular weight. & g/gmol \\
\hline \multirow{5}{*}{ Thermal } & $\operatorname{Tg}$ & Glass transition temperature. & ${ }^{\circ} \mathrm{C}$ \\
\hline & MDT & Maximum degradation temperature. & ${ }^{\circ} \mathrm{C}$ \\
\hline & MFI & Melt flow index. & $\mathrm{g} / 10 \mathrm{~min}$ \\
\hline & THR & Total heat release. & $\mathrm{MJ} / \mathrm{m}^{2}$ \\
\hline & PHRR & Peak heat release rate. & $\mathrm{kW} / \mathrm{m}^{2}$ \\
\hline \multirow{2}{*}{ Mechanical } & $\sigma$ & Maximum stress measured in tensile test. & $\mathrm{MPa}$ \\
\hline & $\varepsilon$ & Maximum elongation observed in tensile test. & $\%$ \\
\hline
\end{tabular}

Table 19. Abbreviations and formulae of some chemical compounds and materials used.

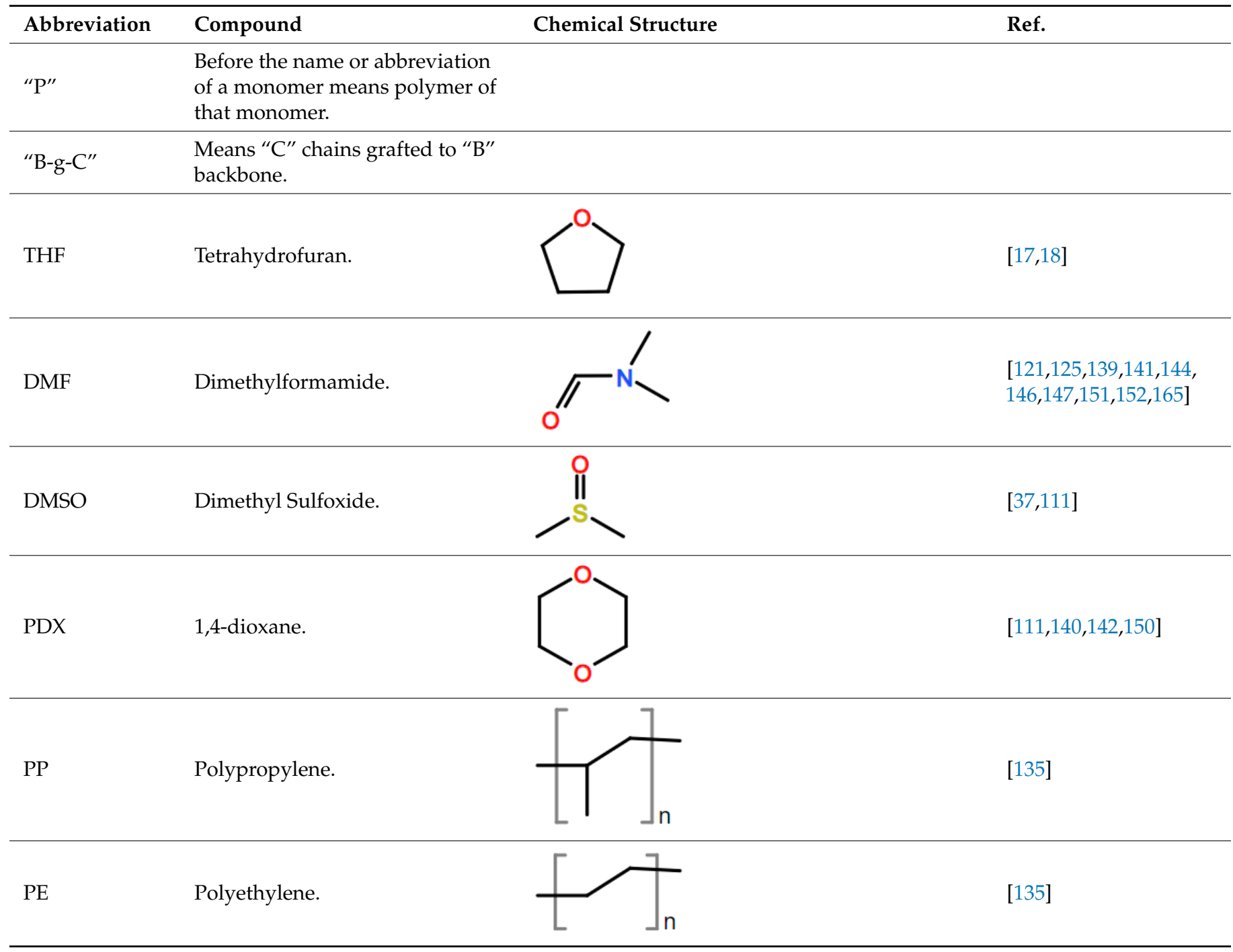


Table 19. Cont.

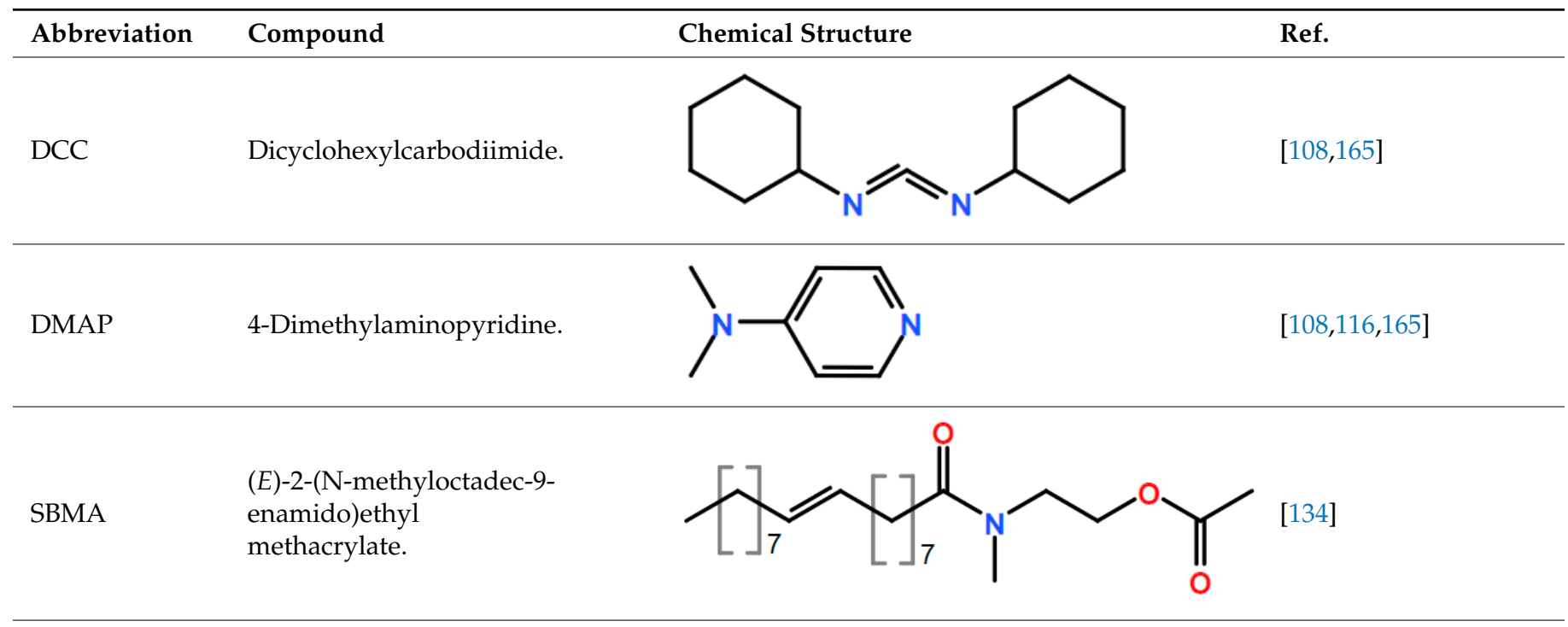

SBMAH

(E)-3-(octadec-9-enamido)propyl methacrylate.<smiles>COCCCNC(=O)C12CCC(/C=C3\[I-]CC3(C)CC1)C[Te]2</smiles>

2-(N-methyl-8-(3-octyloxiran-2-

SBMAEO yl)octanamido)ethyl methacrylate.

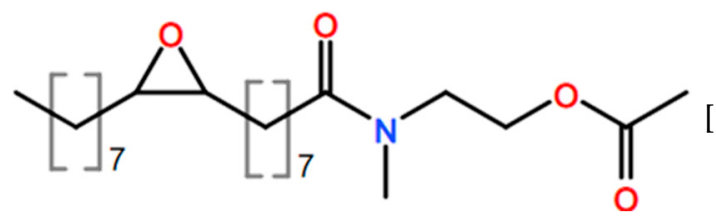

4-cyano-4-

(phenylcarbonothioylthio) pentanoic acid.<smiles>CC(C#N)(CCC(=O)O)SC(=S)c1ccccc1</smiles>

$\mathrm{POCl}_{3}$

Phosphoryl trichloride.<smiles>O=P(Cl)(Cl)Cl</smiles><smiles>O=P12OP3(=O)OP(=O)(O1)OP(=O)(O2)O3</smiles> 
Table 19. Cont.

\begin{tabular}{llll}
\hline Abbreviation & Compound & Ref. & \\
Imidazole. & \\
IPG & $\begin{array}{l}\text { (1H-imidazol-1-yl) phosphonic } \\
\text { group. }\end{array}$
\end{tabular}

N'N'-MBA N'N'-methylenebisacrylamide.<smiles>C=CC(=O)NCNC(=O)C=C</smiles>

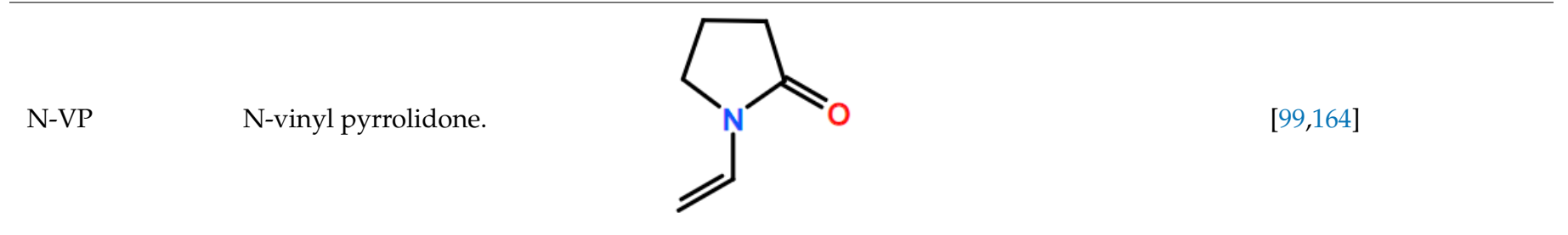

$\mathrm{Co}(\mathrm{acac})_{3} \quad$ Cobaltacetylacetonate complex.

$[99,103]$

MMA

Methyl methacrylate.<smiles>C=C(C)C(=O)OC</smiles>

$[58,107,111,117,140$,

PMMA

Poly(methyl methacrylate).

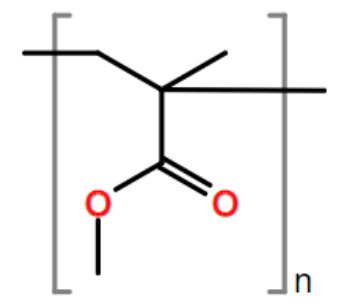

$[58,107,111,117,140$, $144,146,149]$

AcN Acrylonitrile.


Table 19. Cont.

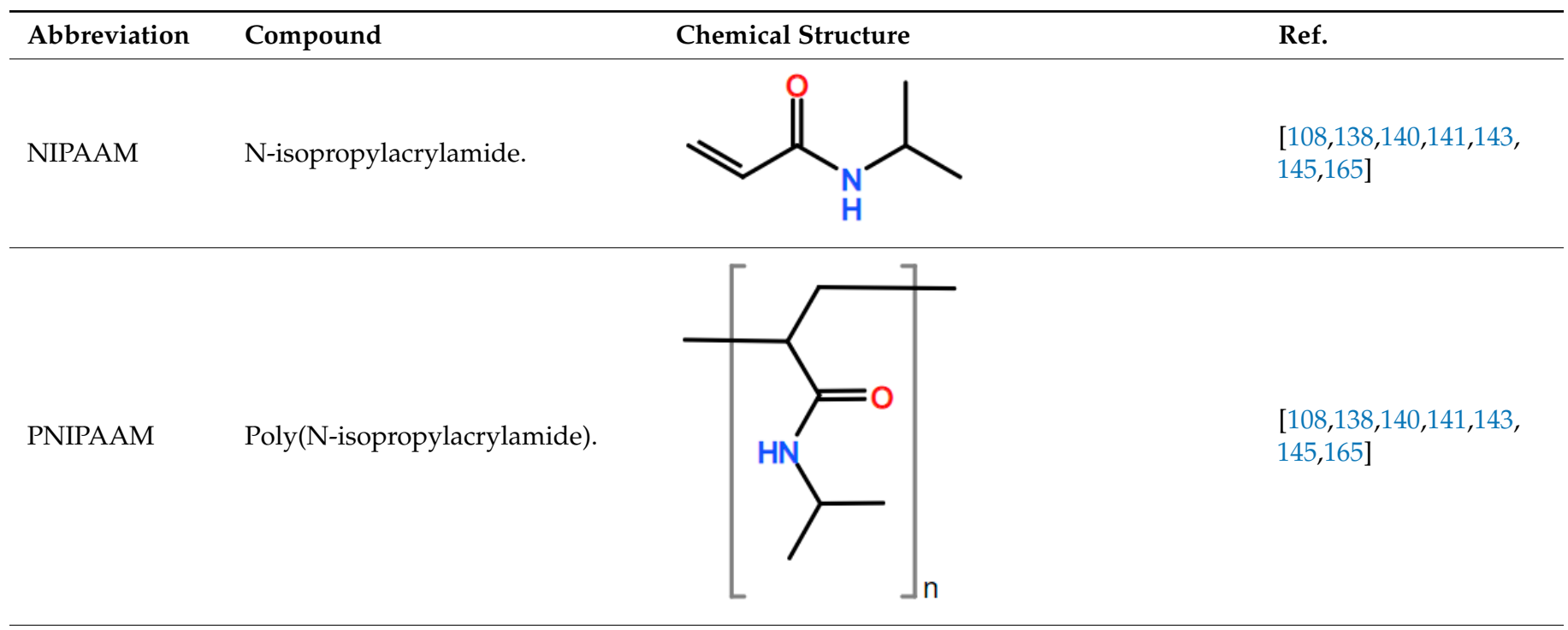

PABTC

Propionic acidyl butyl trithiocarbonate.<smiles>CCCCSC(=S)SC(C)C(=O)O</smiles>

[108]

HPC

Hydroxypropyl cellulose.

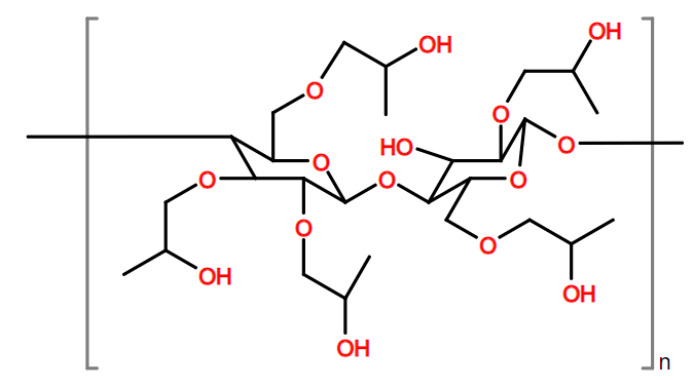

[108]

DMAc

Dimethylacetamide.<smiles>CC(=O)N(C)C</smiles>

[108]

$\begin{array}{lll}\text { CellClAc } & \text { Cellulose chloroacetate. } \\ \text { [109,122] } & \text { 2,2'-bipyridine. }\end{array}$

4NPA

N-(4-nitrophenyl) acrylamide.

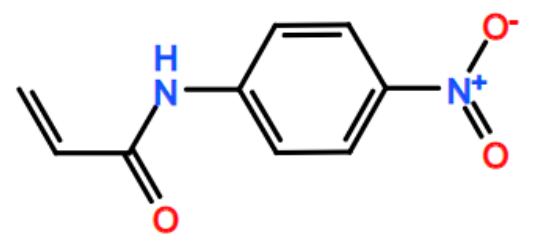

[109]

MA

Methyl acrylate.<smiles>C=CC(=O)OC</smiles> 
Table 19. Cont.

\begin{tabular}{|c|c|c|c|}
\hline Abbreviation & Compound & Chemical Structure & Ref. \\
\hline FAS & Ferrous ammonium sulphate. & $\left(\mathrm{NH}_{4}\right)_{2} \mathrm{Fe}\left(\mathrm{SO}_{4}\right)_{2} 6 \mathrm{H}_{2} \mathrm{O}$ & [110] \\
\hline KPS & Potassium persulphate. & $\mathrm{K}^{+}$ & [110] \\
\hline CAN & Ceric ammonium nitrate. & & [111] \\
\hline
\end{tabular}

$\mathrm{BPO}$

Benzoyl peroxide.<smiles>O=C(OOC(=O)c1ccccc1)c1ccccc1</smiles>

\section{[111]}<smiles>CCCCC(CC)C(=O)O[SnH3]</smiles>
$\mathrm{Sn}(\mathrm{Oct})_{2}$ Tin(II) 2-ethyl hexanoate.

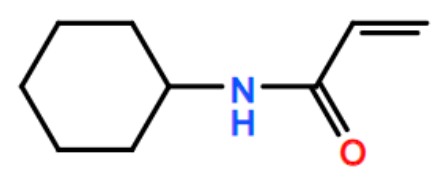

NCA N-cyclohexylacrylamide.
$\mathrm{Ag}$ [113]

\begin{tabular}{|c|c|c|}
\hline AgNPs & Silver nanoparticles. & $\mathrm{Ag}$ \\
\hline$b-C D$ & $\beta$-cyclodextrin. & \\
\hline
\end{tabular}


Table 19. Cont.

\begin{tabular}{|c|c|c|c|}
\hline Abbreviation & Compound & Chemical Structure & Ref. \\
\hline MTC-b-CD & $\begin{array}{l}\text { Monochlorotriazinyl- } \beta \text { - } \\
\text { cyclodextrin. }\end{array}$ & $\mathrm{CD}=$ beta $\mathrm{Cicl}$ & {$[113,114]$} \\
\hline APS & Ammonium persulfate. & & {$[115,117]$} \\
\hline AmimCl & $\begin{array}{l}\text { 1-allyl-3-methylimidazolium } \\
\text { chloride. }\end{array}$ & & [116] \\
\hline PBS & $\begin{array}{l}\text { Phosphate-buffered saline } \\
\text { solutions. }\end{array}$ & & [116] \\
\hline DMAc & N,N-dimethyl acetamide. & & {$[111,117]$} \\
\hline $\mathrm{Br}-\mathrm{iBuBr}$ & 2-Bromoisobutyryl bromide. & & [118] \\
\hline $\begin{array}{l}\text { L-LA } \\
\text { D-LA }\end{array}$ & $\begin{array}{l}\text { L-lactide. } \\
\text { D-lactide. }\end{array}$ & & {$[116,119,157,159]$} \\
\hline PLLA & Poly(L-lactide). & & {$[116,119,157,159]$} \\
\hline PDLA & Poly(D-lactide). & & \\
\hline
\end{tabular}


Table 19. Cont.

$\left.\begin{array}{lll}\hline \text { Abbreviation } & \text { Compound } & \text { Ref. } \\ \text { STY or Sty } & \text { Styrene. } \\ \text { PS or PSty } & \text { Polystyrene. } & \text { [58,118,140,146,147, } \\ 149]\end{array}\right]$

PMDETA

$\mathrm{N}, \mathrm{N}, \mathrm{N}^{\prime}, \mathrm{N}^{\prime}, \mathrm{N}^{\prime \prime}-$

Pentamethyldiethylenetriamine.

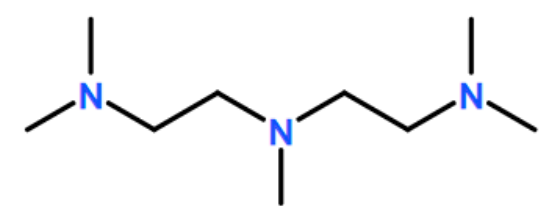

[118,140,146]

PHA

Polyhydroxyalkanoate.

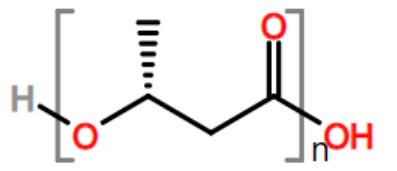

[120]

Poly(hydroxyalkanoate-grafted-

PHA-g-MA

maleic

anhydride).

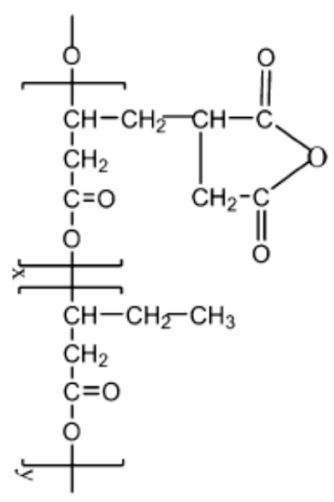<smiles>C1CN=C2NCCCN2C1</smiles>

NCHA N-cyclohexylacrylamide.<smiles>C=CC(=O)NC1CCCCC1</smiles> 
Table 19. Cont.

\begin{tabular}{|c|c|c|c|}
\hline Abbreviation & Compound & Chemical Structure & Ref. \\
\hline CL & $\varepsilon$-Caprolactone.. & & {$[37,158,159,161,162]$} \\
\hline PCL & 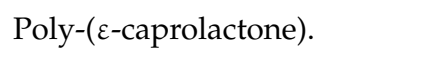 & & {$[37,158,159,161,162]$} \\
\hline $4 \mathrm{VP}$ & 4-vinylpyridine. & & {$[122,164]$} \\
\hline DAAM & Diacetone acrylamide. & & [122] \\
\hline DA & Diallylamine. & & [122] \\
\hline PETA & Pentaerythritol triacrylate. & & [123] \\
\hline TEMPO & TEMPO nitroxide. & & [124] \\
\hline HEMA & 2-hydroxyethyl methacrylate. & & [124] \\
\hline DMAEMA & $\begin{array}{l}\text { 2-dimethylaminoethyl } \\
\text { methacrylate. }\end{array}$ & & {$[138,150]$} \\
\hline
\end{tabular}


Table 19. Cont.

\begin{tabular}{|c|c|c|c|}
\hline Abbreviation & Compound & Chemical Structure & Ref. \\
\hline BA & Butyl acrylate. & & [138] \\
\hline EG & Ethylene glycol. & & [138] \\
\hline $\mathrm{AM}$ & Acrylamide. & & {$[58,138,139,151,153]$} \\
\hline AA & Acrylic Acid. & & {$[58,138,139]$} \\
\hline $\mathrm{ACX}$ & Acyl chloride xanthate. & & {$[139,153]$} \\
\hline LMA & Lauryl methacrylate. & & [140] \\
\hline & Guaiacol. & & [140] \\
\hline & Vainillin. & & [140] \\
\hline
\end{tabular}


Table 19. Cont.

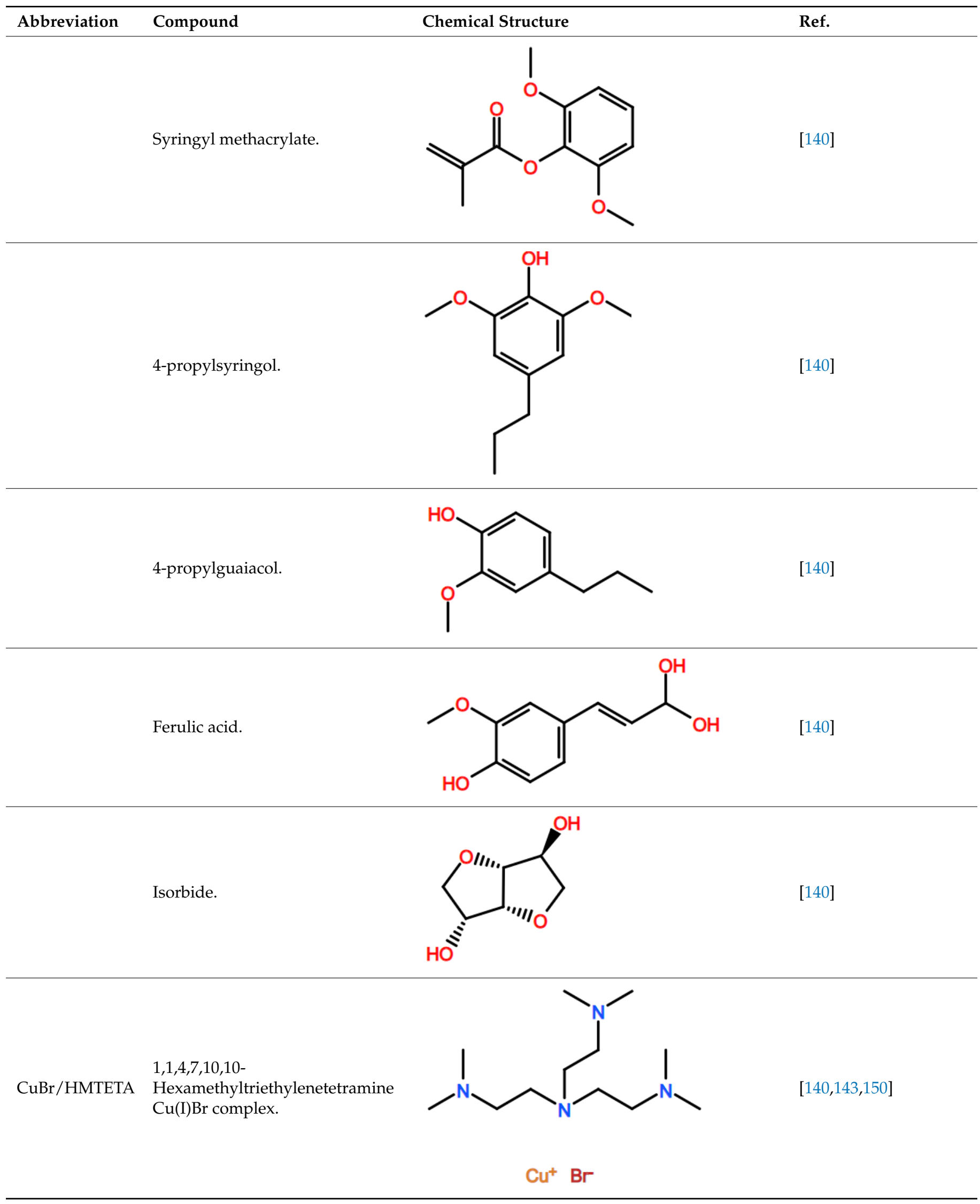


Table 19. Cont.

\begin{tabular}{lll}
\hline Abbreviation & Compound & Ref. \\
$\mathrm{CuBr} / \mathrm{PMDETA}$ & $\begin{array}{l}\mathrm{N}, \mathrm{N}_{1} \mathrm{~N}^{\prime}, \mathrm{N}^{\prime \prime}, \mathrm{N}^{\prime \prime}- \\
\mathrm{Pentamethyldiethylenetriamine} \\
\mathrm{Cu}(\mathrm{I}) \mathrm{Br} \text { complex. }\end{array}$
\end{tabular}

$\begin{array}{ll}\text { PEG-A } & \text { Poly(ethylene glycol) acrylate.n = }\end{array}$<smiles>C=CC(=O)OCCO[Tl]</smiles>
Poly(propylen glycol $)$ acrylate. $n=$ 5<smiles>C=CC(=O)OCC(C)O[TlH]</smiles>

DAEA

Dehydroabietic ethyl acrylate.<smiles>C=CC(=O)OCCOC(=O)C1(C)CCCC2(C)c3ccc(C(C)C)cc3CCC12</smiles>

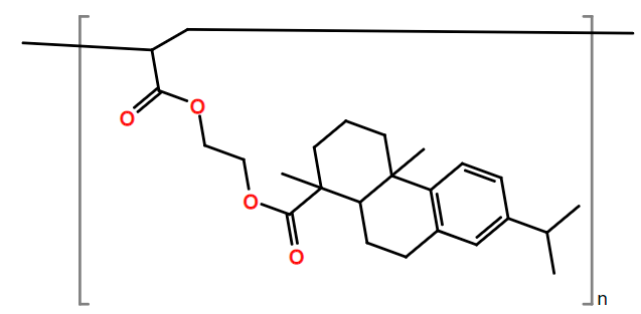

[140,142] 
Table 19. Cont.

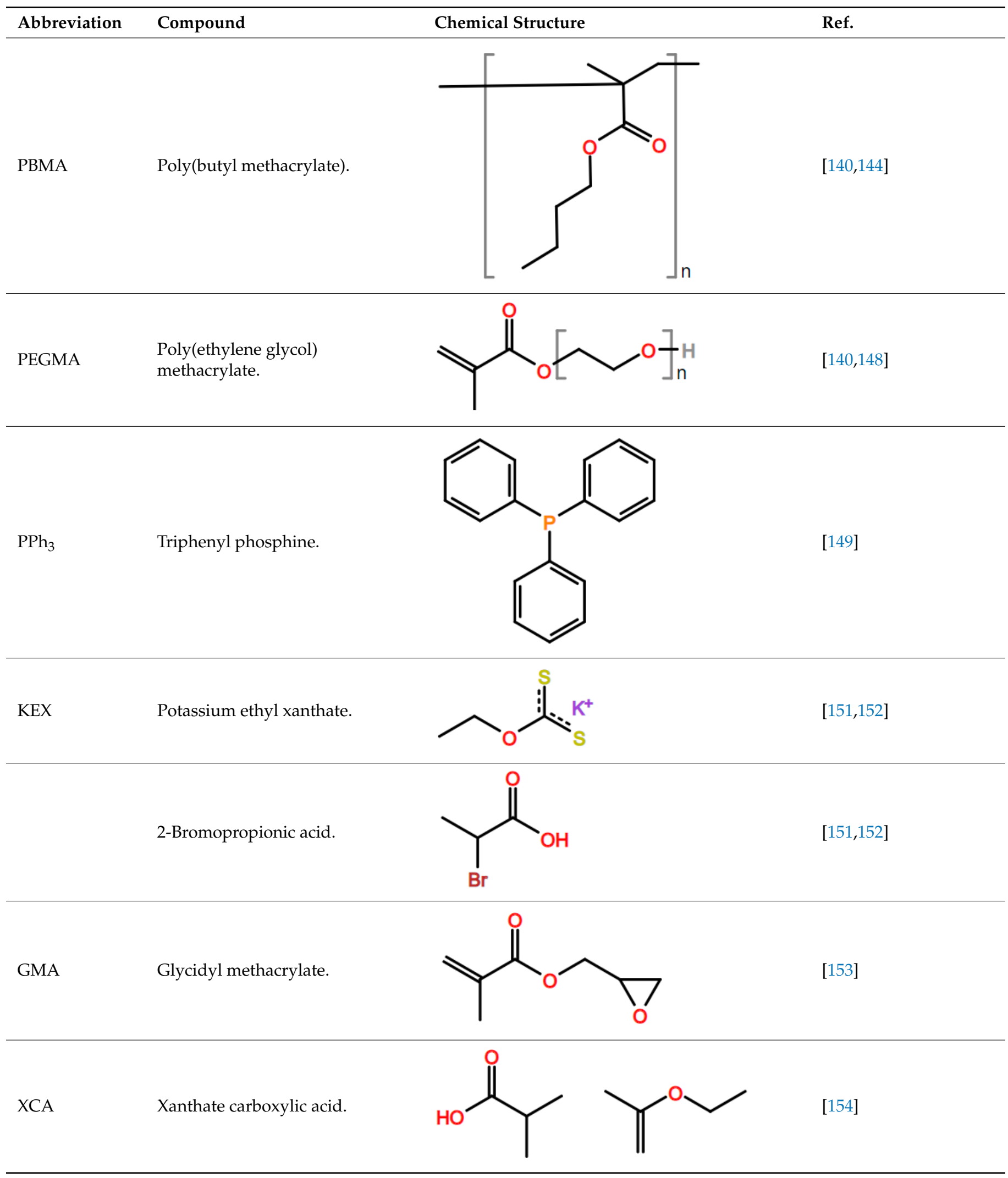


Table 19. Cont.

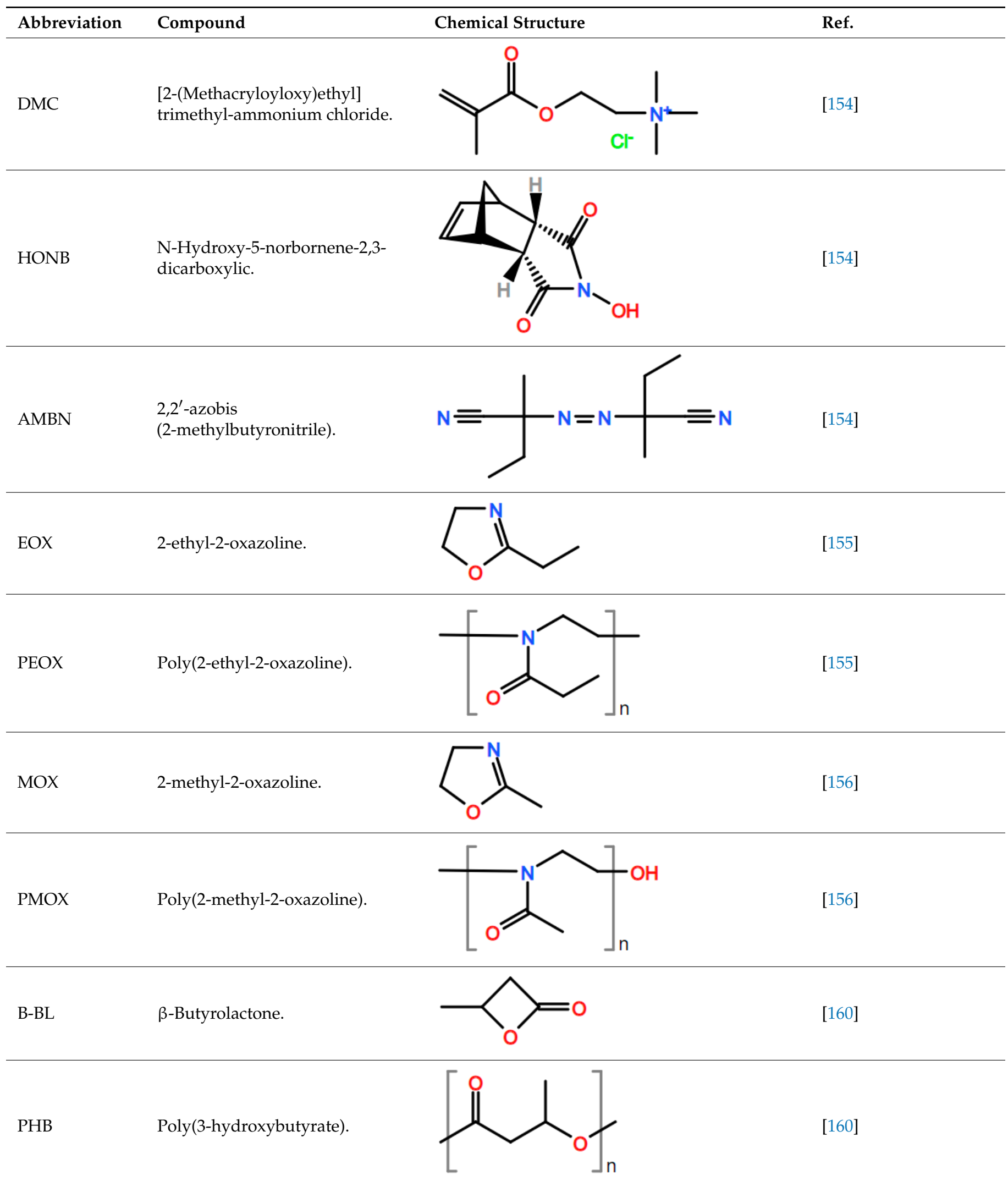


Table 19. Cont.

\begin{tabular}{llll}
\hline Abbreviation & Compound & Ref. \\
\hline EOX & Ethylene oxide. & [163] \\
\hline PEO & Poly(ethylene oxide). & [161] \\
\hline P4-t-Bu & $\begin{array}{l}\text { 1-tert-butyl-4,4,4-tris } \\
\text { (dimethylamino-2,2- } \\
\text { bis[tris(dimethylamino)- } \\
\text { phosphoranylidenamino]-215,415- } \\
\text { catenadi (phosphazene) solution } \\
\text { in hexane. }\end{array}$ & {$[163]$} \\
\hline
\end{tabular}

\section{Characterization Techniques Used for Polymer Grafted Materials}

The characterization of polymer grafted materials requires the use of a variety of methods due to the many possible combinations of backbones and polymer grafts [59]. The characterization methods can be classified as direct or indirect.

Direct methods are those used to identify changes in the chemical structure of grafted materials, such as the bonds between backbone and grafts. These methods include proton and carbon magnetic nuclear resonance, ${ }^{1} \mathrm{H}-\mathrm{NMR}$, and ${ }^{13} \mathrm{C}-\mathrm{NMR}$, respectively.

Indirect methods are based on differences in properties between the starting and grafted materials, relating these changes to the modified structures. Microscopy and thermal analysis are examples of indirect methods. A summary of the main characterization methods used for grafted materials is presented in Table 6.

The relevant information contained in selected articles is also gathered in this review to show how the characterization techniques were used to provide evidence of polymer grafting onto the corresponding backbones. Tables 7-12 summarize the use of thermal, spectroscopic, imaging and microscopy, rheological, chromatographical, and mechanical characterization techniques, respectively, in the analysis of polymer grafted materials. Finally, a summary of biological, functional, and composition characterization techniques used for grafted materials is provided in Table 13.

\section{Modeling of Polymer Grafting}

\subsection{Literature review on Modeling of Polymer Grafting}

An overview of the literature on the modeling of polymer grafting is summarized in Table 14. The backbones considered, the functionalization methods, the polymer chains grafted, and summary comments on the modeling approaches used to carry out the simulations are included in the table.

\subsection{Modeling of Polymer Branching and Crosslinking}

As observed in Table 14, most reports on the modeling of polymer grafting are related to cases where grafting involves free-radical growth of the grafts, and the generation of active sites proceeds through chain transfer to polymer reactions. In that sense, the growth of polymer grafts resembles the formation and growth of branches in polymer branching. The difference would be that the branches and primary polymer chains contain the same monomers, whereas grafts and backbones contain different monomers in polymer grafting. There are several papers focused on the modeling of polymer branching [262-268]. In some cases, as in the grafting of monochlorotriazinyl- $\beta$-cyclodextrin onto cellulose, the activation mechanism is not specified, and the modeling approach is fully empirical (neural network modeling) [237-239]. 
In general terms, the polymerization scheme of FRP including chain transfer to polymer (CTP) is given by the reactions shown in Table 15. The specific mathematical expressions containing CTP terms are given by Equations (1)-(7). I, R, and M in Table 15 are initiator, primary free radical, and monomer molecules, respectively; $P_{n}$ and $D_{m}$ denote live and dead polymer molecules, respectively, of sizes $n$ and $m . k_{i}, k_{p}, k_{t d}$, and $k_{\text {trp }}$ (also denoted as $k_{\mathrm{fp}}$ in Equations (8) and (9)) denote initiation, propagation, termination by disproportionation, and chain transfer to polymer kinetic rate constants, respectively.

Polymer branching can be modeled using a bivariate distribution of chain length and number of branches resulting from polymerizations involving branched polymers [269]. $P_{n, b}$ in Table 15 accounts for a bivariate distribution of live polymer of length $n$ and number of branches $b$. The moment equations shown below consider only the kinetic steps of propagation and chain transfer to polymer, for illustrative purposes. For a batch reactor, the application of the mass action law considering only these two kinetic steps results in Equation (1) [269]. It should be noticed that in the transfer to polymer reaction there are as many possible sites of reaction as monomeric units in the dead polymer chain participating in the reaction.

$$
\begin{gathered}
\frac{d P_{n, b}}{d t}=\ldots-k_{p}\left(P_{n, b} M+P_{n-1, b} M\right)-\mathrm{k}_{\operatorname{trp}} P_{n, b}\left(\sum_{m=1}^{\infty} \sum_{c=0}^{\infty} m D_{m, c}\right)+\mathrm{k}_{\operatorname{trp}} n D_{n, b-1} \sum_{h=1}^{\infty} \sum_{e=0}^{\infty} P_{h, c}+\ldots \\
n=1, \ldots, \infty ; b=0, \ldots, \infty
\end{gathered}
$$

The bivariate moments for active and inactive polymer are defined respectively as shown in Equations (2) and (3). Number and weight-averaged molecular weights, and the average number of branches, are given by Equations (4)-(6) [269].

$$
\begin{gathered}
\mu_{G, H}=\sum_{n=1}^{\infty} \sum_{b=0}^{\infty} n^{G} b^{H} P_{n, b} \\
\lambda_{G, H}=\sum_{n=1}^{\infty} \sum_{b=0}^{\infty} n^{G} b^{H} D_{n, b} \\
M_{n}=\frac{\mu_{1,0}+\lambda_{1,0}}{\mu_{0,0}+\lambda_{0,0}} W_{m} \\
M_{w}=\frac{\mu_{2,0}+\lambda_{2,0}}{\mu_{1,0}+\lambda_{1,0}} W_{m} \\
B_{n}=\frac{\mu_{0,1}+\lambda_{0,1}}{\mu_{0,0}+\lambda_{0,0}}
\end{gathered}
$$

The moment equations for live polymer are given by Equation (7) [269].

$$
. \frac{d \mu_{G, H}}{d t} . .-k_{p} M \mu_{G, H}+k_{p} M \sum_{R=0}^{G}\left(\begin{array}{c}
G \\
R
\end{array}\right) \mu_{G-R, H}-k_{t r p} \mu_{G, H} \lambda_{1,0}+k_{t r p} \mu_{0,0} \sum_{K=0}^{H}\left(\begin{array}{c}
H \\
K
\end{array}\right) \lambda_{G+1, H}+\ldots
$$

Another approach with which to address the modeling of polymer branching in FRP is to use the concept of branching density, denoted as $\rho$, which is given by the ratio of the number of branching points to that of monomeric units, and it can be estimated using Equation (8), which when solved leads to Equation (9) [270]. $\mathrm{k}_{\mathrm{fp}}$ and $\mathrm{k}_{\mathrm{p}}$ in Equations (8) and (9) are chain transfer to polymer and propagation kinetic rate constants, respectively, and $\mathrm{x}$ is monomer conversion.

$$
\begin{gathered}
\frac{d(x \rho)}{d x}=\frac{k_{f p} x}{k_{p}(1-x)} \\
\beta=-\frac{k_{f p}}{k_{p}}\left[1+\frac{\ln (1-x)}{x}\right]
\end{gathered}
$$

CTP and terminal double bond (TDB) polymerization produce tri-functional (long) branches, in addition to increasing the weight-averaged molecular weight and broadening the MWD. A reaction "similar" to TDB polymerization is the polymerization with internal 
(pendant) double bonds (double bonds "internal" in dead polymer chains, appearing therein due to (co)polymerization of di-functional (divinyl) monomers (e.g., butadiene). Internal double bond (IDB) polymerization produces tetra-functional (long) branches and leads eventually to the formation of crosslinked polymer (gel). Both molecular weight averages increase due to IDB polymerization and the MWD broadens considerably [43]. Crosslinking can be considered as interconnected branching, and in that sense, its growth by CTP and its modeling in terms of a crosslink density, denoted as $\rho_{a}$, can also be taken as a useful basis for the modeling of polymer grating by CTP and propagation through the intermediate free radicals. Balance equations for polymer radicals of size $r\left(R_{r}^{*}\right)$ and $\rho_{\mathrm{a}}$ for a case of copolymerization with crosslinking of vinyl/divinyl monomers, using the pseudo-kinetic rate constants method, are given by Equations (10) and (11), respectively, where $P_{s}$ is a dead polymer of size $r$; $Q_{1}$ is first-order moment for the dead polymer; and $\mathrm{k}_{\mathrm{cp}}$ and $\mathrm{k}_{\mathrm{cs}}$ are primary and secondary cyclization rate constants, respectively [271].

$$
\begin{gathered}
\frac{1}{\nabla} \frac{\mathrm{d}\left(\mathrm{V}\left[\mathrm{R}_{\mathrm{r}}^{*}\right]\right)}{\mathrm{dt}}=\mathrm{k}_{\mathrm{p}}[\mathrm{M}]\left[\mathrm{R}_{\mathrm{r}-1}^{*}\right]+\mathrm{k}_{\mathrm{f} \mathrm{p}} \mathrm{r}\left[\mathrm{P}_{\mathrm{r}}\right]\left[\mathrm{R}^{*}\right]+\mathrm{k}_{\mathrm{p}}^{*} \sum_{\mathrm{s}=1}^{\mathrm{r}-1} \mathrm{~s}\left[\mathrm{R}_{\mathrm{r}-\mathrm{s}}^{*}\right]\left[\mathrm{P}_{\mathrm{s}}\right] \\
-\left(\mathrm{k}_{\mathrm{p}}^{*}\right)[\mathrm{M}]\left[\mathrm{R}_{\mathrm{r}}^{*}\right]-\left(\mathrm{k}_{\mathrm{td}}+\mathrm{k}_{\mathrm{tc}}\right)\left[\mathrm{R}^{*}\right]\left[\mathrm{R}_{\mathrm{r}}^{*}\right]-\left(\mathrm{k}_{\mathrm{p}}^{*}+\mathrm{k}_{\mathrm{fp}}\right) \mathrm{Q}_{1}\left[\mathrm{R}_{\mathrm{r}}^{*}\right] \\
\frac{\mathrm{d}\left[\mathrm{x} \overline{\rho_{\mathrm{a}}}\right]}{\mathrm{dt}}=\frac{\mathrm{k}_{\mathrm{p}}^{*}\left[\overline{\mathrm{F}_{2}}\left(1-\mathrm{k}_{\mathrm{cp}}\right)-\overline{\rho_{\mathrm{a}}}\left(1+\mathrm{k}_{\mathrm{cs}}\right)\right] \mathrm{x}}{\mathrm{k}_{\mathrm{p}}(1-\mathrm{dx})} \frac{\mathrm{dx}}{\mathrm{dt}}
\end{gathered}
$$

\subsection{Main Modeling Equations for Polymer Grafting}

Grafting efficiency $(\varepsilon)$ and number average molecular weights for the different polymer populations $\left(\mathrm{W}_{\mathrm{IH}}, \mathrm{W}_{\mathrm{SH}}, \mathrm{W}_{\mathrm{II}}, \mathrm{W}_{\mathrm{IS}}\right.$, and $\left.\mathrm{W}_{\mathrm{SS}}\right)$ for the grafting of vinyl polymers onto pre-formed polymer with highly active chain transfer sites of (pendant mercaptan groups) are given by Equations (12)-(17) [39]. Subscripts IH and SH in the molecular weights shown in Equations (12)-(17) account for primary chains formed by chain transfer or by disproportionation termination (without distinguishing between terminally saturated and unsaturated chains) of polymer radicals starting with I and S fragments, respectively. Subscripts II, IS, and SS, on the other hand, account for primary chains produced by combination of the appropriate pair of polymer radicals starting with I and S fragments, respectively. $\mathrm{r}$ in Equations (12)-(18) is the ratio of propagation to termination kinetic rate constants, namely, $\mathrm{r}=\mathrm{k}_{\mathrm{p}} / 2 \mathrm{k}_{\mathrm{t}}$.

$$
\begin{gathered}
\varepsilon=\frac{W_{S H}+W_{S S}+W_{I S}}{W_{S H}+W_{S S}+W_{I S}+W_{I H}+W_{I I}} \\
W_{I H}=m[M]_{0} \int_{0}^{\propto}\left\{\frac{y_{0}}{y_{0}+(1-\alpha)^{C_{S}}}-\frac{(1-r) y_{0}^{2}}{\left[y_{0}+(1-\alpha)^{C_{S}}\right]^{2}}\right\} \mathrm{d} \propto \\
W_{S H}=m[M]_{0} \int_{0}^{\propto}\left\{\frac{(1-\propto)^{C_{S}}}{y_{0}+(1-\alpha)^{C_{S}}}-\frac{(1-r) y_{0}(1-\alpha)^{C_{S}}}{\left[y_{0}+(1-\alpha)^{C_{S}}\right]^{2}}\right\} \mathrm{d} \propto \\
W_{I I}=m[M]_{0} \int_{0}^{\alpha}\left\{\frac{(1-r) y_{0}^{3}}{\left[y_{0}+(1-\propto)^{C_{S}}\right]^{3}}\right\} \mathrm{d} \propto \\
W_{I S}=2 m[M]_{0} \int_{0}^{\propto}\left\{\frac{(1-r) y_{0}^{2}(1-\alpha)^{C_{S}}}{\left[y_{0}+(1-\propto)^{C_{S}}\right]^{3}}\right\} \mathrm{d} \propto
\end{gathered}
$$




$$
W_{S S}=m[M]_{0} \int_{0}^{\alpha}\left\{\frac{(1-r) y_{0}(1-\alpha)^{2 C_{S}}}{\left[y_{0}+(1-\alpha)^{C_{S}}\right]^{3}}\right\} \mathrm{d} \propto
$$

An example of calculation of the mole fraction chain length distribution (number distribution) $\left(n_{x}\right)$ for the case of polymer grafting of vinyl polymers onto solid polymeric substrates, considering no chain transfer and incomplete conversion, is shown in Equation (18) [40].

$$
n_{x, 1}=\frac{\left[R_{0}\right]}{r[M]_{0}\left\{1-\left(\frac{[M]}{[M]_{0}}\right)^{1 / r}\right\}}\left\{1+\frac{\left[R_{0}\right](1-r) x}{r[M]_{0}}\right\}^{\frac{r-2}{1-r}}
$$

When addressing the modeling of polymer grafting of vinyl polymer onto polyolefins in extruders by CTP, Hamielec et al. [231] proposed a polymerization scheme and the corresponding kinetic equations where the prepolymer molecule bears abstractable hydrogens on its backbone and a second compound, denoted as additive (A), is bound to the prepolymer backbone via reaction with a free radical. The backbone radical then transfers its radical center to the active molecules. The radical is finally terminated with other radicals. Proper kinetic equations were written down for the participating species, and a degree of grafting, $\mathrm{g}$, which is the average of number of grafted molecules per monomer unit on the prepolymer backbone, was defined as shown in Equation (19), where $Q_{1}$ is the concentration of monomer units on prepolymer backbones which remains constant during branching, $\mathrm{K}_{\mathrm{a}, 3}$ is the kinetic coefficient for the grafting (additive addition) reaction, and $\mathrm{R}_{03}$ designates a primary radical with radical center on backbone $\mathrm{R}_{0}$. Further mathematical treatment by the authors leads to Equation (20) for calculation of the full chain length distribution of the polymer population, $\mathrm{w}(\mathrm{r}, \mathrm{s})$, where $\mathrm{w}_{0}(\mathrm{r})$ is the initial chain length distribution of the prepolymer [231].

$$
\begin{gathered}
\frac{d g}{d t}=\frac{K_{a, 3} R_{0,3} A}{Q_{1}} \\
w(r, s)=\left(1+\frac{s}{r}\right) \frac{w_{0}(r)}{(1+g) s !}(g r)^{s} e^{-g r}
\end{gathered}
$$

As stated above in Table 14, Gianoglio Pantano et al. [245] developed a very detailed model for grafting of PSty onto PE. Among the concentrations of species calculated by the model, the concentration of poly(ethylene-g-styrene) is calculated using Equation (21), and the concentration of grafted PS, denoted as Gr, is obtained from Equation (22). G(t) is a matrix array of "infinite" size whose elements contain the molar concentrations of the individual species with degree of polymerization indicated by its subscripts. VG is a vector that contains the corresponding reaction rate terms, $\mathrm{I}_{1,0}$ is a bivariate moment of order 1 for $\mathrm{PS}$ and order 0 for $\mathrm{PE}$, which represents the mass of PS grafted onto $\mathrm{PE}$, and $\mathrm{M}^{\mathrm{PS}}{ }_{1}$ is the molar mass of PS.

$$
\begin{gathered}
\frac{d \boldsymbol{G}(t)}{d t}=V G ; G(\mathbf{0})=\mathbf{0} \\
G r=100 \frac{I_{1,0}}{M_{1}^{P S}(t=0)}
\end{gathered}
$$

Very detailed simulation studies for the grafting of vinyl polymers onto PE using kMC were presented by Hernández-Ortiz et al. [41,259-261]. Some of the key reactions considered in this study are shown in Figure 7 and the modeling strategy is summarized in Figure 8 [259]. 


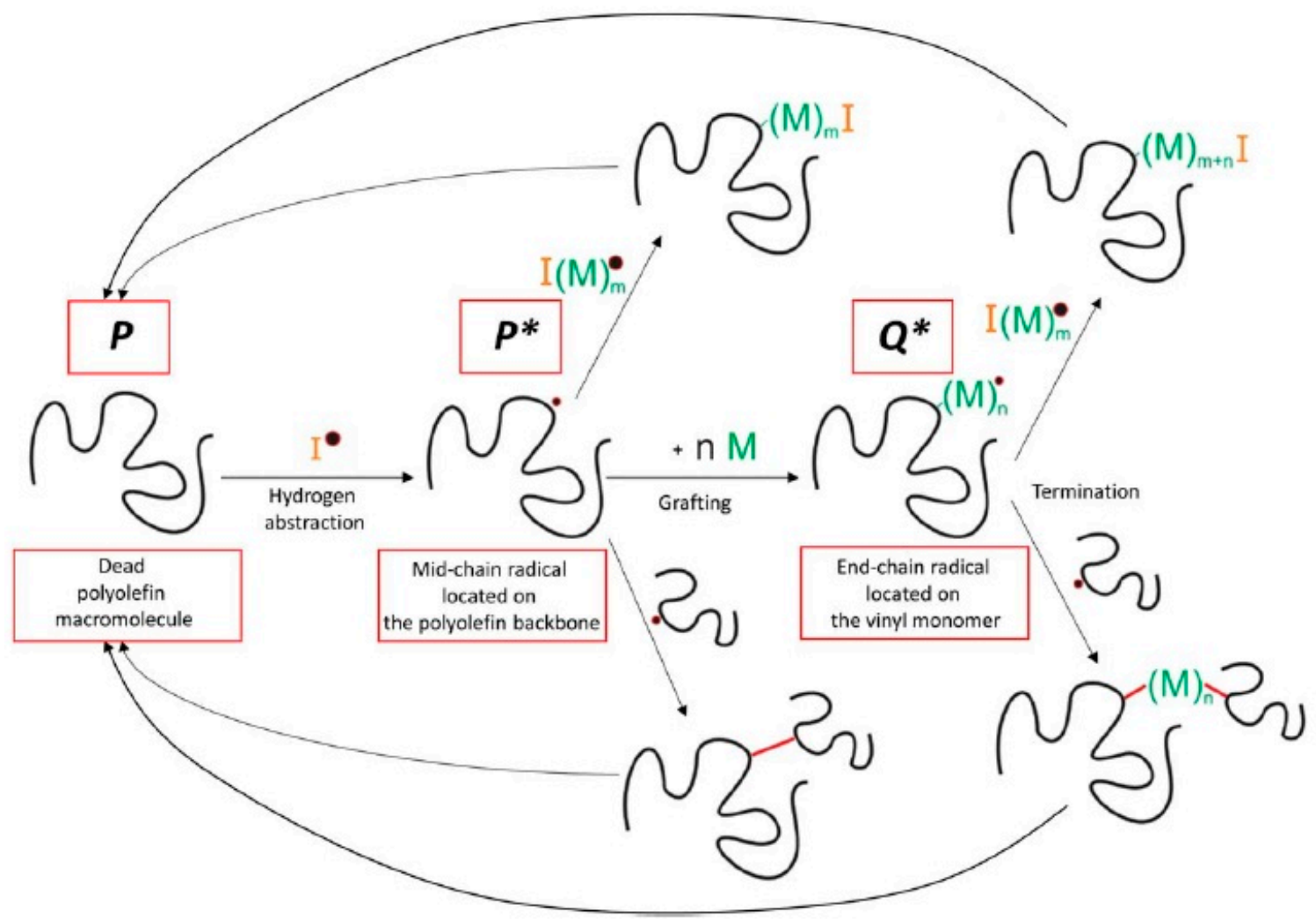

Figure 7. Some of the key reactions present in the grafting of polyolefins with vinyl monomer M. Reprinted with permission from Hernández-Ortiz et al., AIChE J., 63(11), 4944-4961 [259]. Copyright 2017 John Wiley and Sons, New York. 


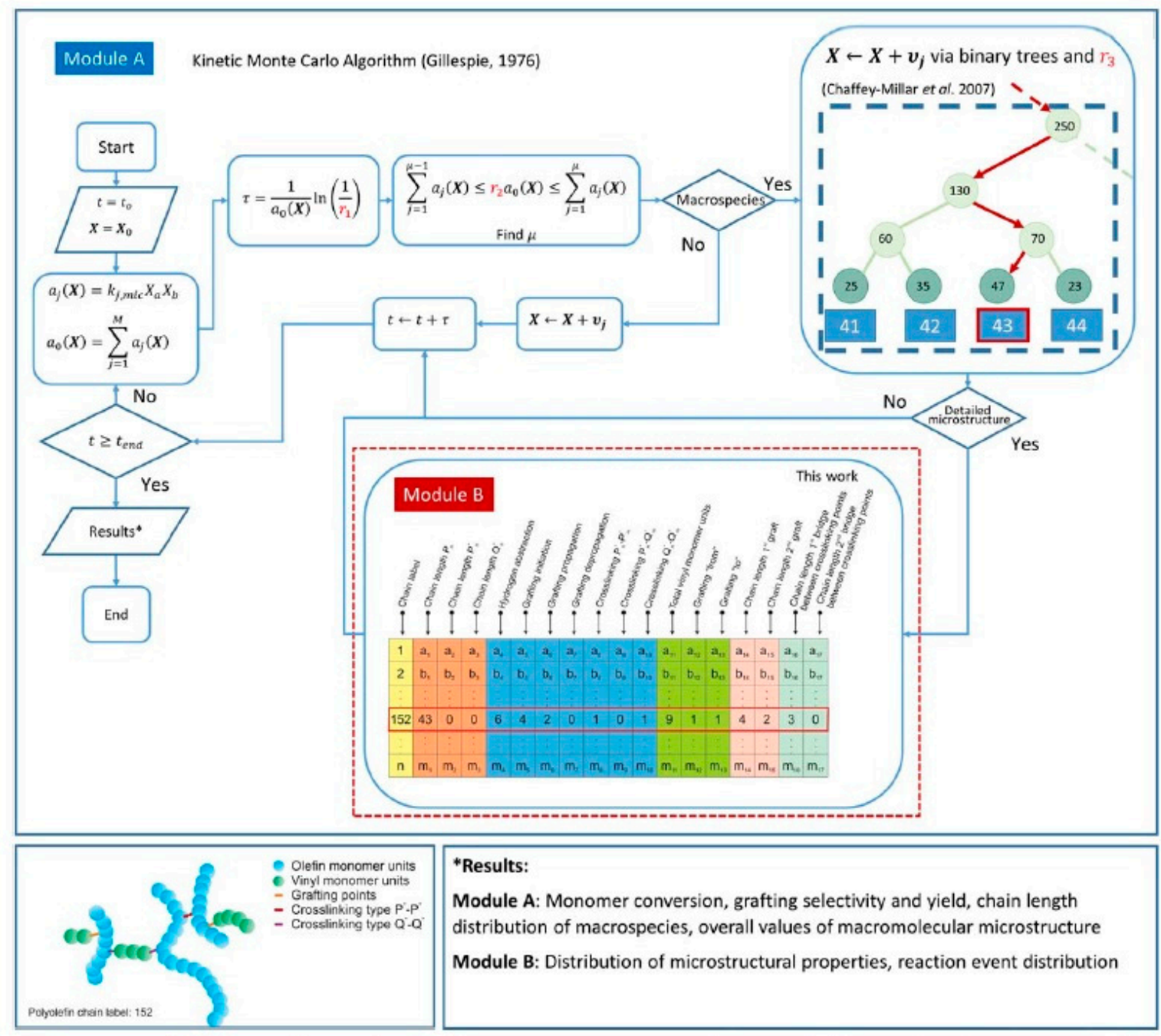

Figure 8. Simulation approach using kMC for the grafting of polyolefins with vinyl monomer M. Reprinted with permission from Hernández-Ortiz et al., AIChE J., 63(11), 4944-4961 [259]. Copyright 2017 John Wiley and Sons, New York.

\section{Nomenclature, Symbols, Abbreviations, and Chemical Structures}

As mentioned earlier, symbols and abbreviations used in this contribution are defined and summarized in Tables 16-19.

\section{Conclusions}

Polymer grafting is a useful route for the synthesis of materials with interesting mechanical, thermal, dilute solution, and melt properties, and the ability to be compatible with otherwise incompatible mixtures. Systematic studies on polymer chemistry and characterization, and even modeling studies of polymer grafting started in about the 1950s. The interest in the grafting of natural biopolymers has escalated in the last 20 or so years due to environmental and sustainability issues.

Most of the studies on modeling of polymer grafting have focused on insertion of active sites on the backbone by CTP and growth of grafts by FRP or variants of FRP, such as RDRP. The monomers of major use for polymer grafting purposes are acrylic monomers (MMA, NIPAAM, AM, AA, BA), styrene (STY) and vinyl ethers [140,184]. When fine control of grafted structures is not required, polymer grafting by FRP may be enough. However, when precise control of polymer grafts (size and separation) is required, RDRP techniques, such as NMP, ATRP, and RAFT, are more adequate. RDRP polymer grafting techniques usually proceed by the "grafting-from" route $[140,180,184]$. One disadvantage 
of RDRP techniques is that they require longer reaction times. For instance, polymer grafting by RAFT polymerization lasts from 8 to $48 \mathrm{~h}$, plus the time required to prepare the related microcontrollers, which in many cases includes an esterification step through Steglich or anhydride procedures. Polymer grafting by ATRP takes from $24 \mathrm{~h}$ to several days.

Polymer grafting by ROP procedures using L-lactide (L-LA) and $\varepsilon$-caprolactone (CL) for the synthesis of poly(l-lactic acid) and poly( $\varepsilon$-caprolactone) polymer grafts is gaining importance $[181,182,189]$. Other monomers used are 2-ethyl-2-oxazoline, to produce PEOX, and ethylene glycol, to produce PEG. These reactions are commonly conducted at temperatures higher than $80^{\circ} \mathrm{C}$, which complicates solvent selection, when using metal catalysts such as $\mathrm{Sn}(\mathrm{Oct})_{2}$. Solvents should dissolve monomer and polymer, perform adequately at the selected temperatures, and show "green" characteristics. DMF, DMSO, p-dioxane, and toluene are some of the solvents most commonly used for polymer grafting by ROP. However, the recent advent of metal-free and organocatalyzed ROP has facilitated the polymerization at room temperature. For example, poly(lactide) materials can be made by ROP of L-LA at ambient conditions in the presence of 1,5,7-triazabicyclo[4.4.0]dec-5-ene (TBD) and 1,8-diazabicyclo[5.4.0]undec-7-ene (DBU) [272].

Polymer grafting is also important in the synthesis of "dendrigraft copolymers." A large variety of heterogeneous dendrigraft copolymer architectures with core-shell and core-shell-corona morphologies can be produced, at significantly lower costs than for conventional dendrimer syntheses [18].

As observed in Table 14, the modeling of polymer grafting has focused on CTP or site formation by irradiation with FRP chemistry, in conventional flasks, stirred-tank reactors, or extruders. Other chemical routes have been addressed using semi-empirical approaches only. Therefore, there is still much to do in and contribute to this area.

Author Contributions: E.V.-L. and A.P. conceived the original idea (with discussions with A.R.-A., J.P.-A., M.G.H.-L., and A.M.). E.V.-L., M.G.H.-L., and A.M. assured funding acquisition through a project where experimental and theoretical work on polymer grafting of biopolymers from lignocellulosic biomasses has been carried out and provided background and motivation for this contribution. M.Á.V.-H., G.S.C.-D., A.R.-A., and E.V.-L. carried out critical literature reviews on different topics of the review. M.Á.V.-H., A.R.-A., and E.V.-L. wrote the original draft of the paper; E.V.-L., A.M., A.P., and Y.M. read and corrected different versions of the manuscript and provided extra references and discussion points. All authors have read and agreed to the published version of the manuscript.

Funding: This research was funded by: (a) Consejo Nacional de Ciencia y Tecnología (CONACYT, México), PhD scholarships granted to M.A.V.-H. and G.S.C.-D.; (b) DGAPA-UNAM, Projects PAPIIT IG100718, IV100119, TA100818, and TA102120, granted to E.V.-L.—the first two- and to A.R.-A.- the last two; and PASPA sabbatical support to E.V.-L. while at the University of Waterloo, in Ontario, Canada; (c) Facultad de Química, UNAM, research funds granted to E.V.-L. (PAIP 5000-9078) and A.R.A. (PAIP 5000-9167); (d) NSERC funding to A.P.; and (e) the Department of Chemical Engineering, University of Waterloo, Canada, partial sabbatical support to E.V.-L. with research funds from A.P. No funding was received for APC.

Institutional Review Board Statement: Not applicable.

Informed Consent Statement: Not applicable.

Data Availability Statement: Data sharing is not applicable to this article.

Conflicts of Interest: The authors declare no conflict of interest. The funders had no role in the design of the study; in the collection, analyses, or interpretation of data; in the writing of the manuscript, or in the decision to publish the results.

\section{References}

1. Hadjichristidis, N.; Pitsikalis, M.; Iatrou, H.; Driva, P.; Chatzichris, M.; Sakellariou, G.; Lohse, D. Graft copolymers. In Encyclopedia of Polymer Science and Technology, 2nd ed.; Matyjaszewski, K., Ed.; John Wiley \& Sons: Hoboken, NJ, USA, 2010; pp. 1-38, ISBN 978-047-144-026-0. 
2. Slagman, S.; Zuilhof, H.; Franssen, M.C.R. Laccase-Mediated Grafting on Biopolymers and Synthetic Polymers: A Critical Review. ChemBioChem 2017, 19, 288-311. [CrossRef]

3. Stannett, V.T. Block and graft copolymerization. In Journal of Polymer Science: Polymer Letters, 1st ed.; Ceresa, R.J., Ed.; John Wiley \& Sons: Hoboken, NJ, USA, 1973; Volume 1, pp. 669-670. [CrossRef]

4. Meier, D.J. Theory of block copolymers. I. Domain formation in A-B block copolymers. J. Polym. Sci. C Polym. Symp. 1969, 26, 81-98. [CrossRef]

5. Helfand, E.; Block Copolymer Theory. III. Statistical Mechanics of the Microdomain Structure. Macromolecules 1975, 8, 552-556. [CrossRef]

6. Helfand, E.; Wasserman, Z.R. Block Copolymer Theory. 4. Narrow Interphase Approximation. Macromolecules 1976, 9, 879-888. [CrossRef]

7. Helfand, E. Block copolymers, polymer-polymer interfaces, and the theory of inhomogeneous polymers. Acc. Chem. Res. 1975, 8, 295-299. [CrossRef]

8. Blanchette, J.A.; Nielsen, L.E. Characterization of graft polymers. J. Polym. Sci. 1956, 20, 317-326. [CrossRef]

9. Merret, F.M. Graft polymers with preset molecular configurations. J. Polym. Sci. 1957, 24, 467-477. [CrossRef]

10. Gluckman, M.S.; Kampf, M.J.; O’brien, L.J.; Fox, T.G.; Graham, R.K. Graft copolymers from polymers having pendant mercaptan groups. II. Synthesis and characterization. J. Polym. Sci. 1959, 37, 411-423. [CrossRef]

11. Miller, M.L. Block and graft polymers I. Graft polymers from acrylamide and acrylonitrile. Can. J. Chem. 1957, 36, 303-308. [CrossRef]

12. Beevers, R.B.; White, E.F.T.; Brown, L. Physical properties of vinyl polymers. Part 3.-X-ray scattering in block, random and graft methyl methacrylate + acrylonitrile copolymers. Trans. Faraday Soc. 1960, 56, 1535-1541. [CrossRef]

13. Oster, G.; Oster, G.K.; Moroson, H. Ultraviolet induced crosslinking and grafting of solid high polymers. J. Polym. Sci. 1959, XXXIV, 671-684. [CrossRef]

14. Kobayashi, Y. Gamma-ray-induced graft copolymerization of styrene onto cellulose and some chemical properties of the grafted polymer. J. Polym. Sci. 1961, 51, 359-372. [CrossRef]

15. Bridgeford, D.J. Catalytic Deposition and Grafting of Olefin Polymers into Cellulosic Materials. Ind. Eng. Chem. Prod. Res. Dev. 1962, 1, 45-52. [CrossRef]

16. Huang, R.Y.-M.; Immergut, B.; Immergut, E.H.; Rapson, W.H. Grafting vinyl polymers onto cellulose by high energy radiation. I. High energy radiation-induced graft copolymerization of styrene onto cellulose. J. Polym. Sci. A Gen. Pap. 1963, 1, $1257-1270$. [CrossRef]

17. McManus, N.; Zhu, S.-H.; Tzoganakis, C.; Penlidis, A. Grafting of ethylene-ethyl acrylate-maleic anhydride terpolymer with amino-terminated polydimethylsiloxane during reactive processing. J. Appl. Polym. Sci. 2006, 101, 4230-4237. [CrossRef]

18. Cadena, L.-E.; Gauthier, M. Phase-segregated dendrigraft copolymer architectures. Polymers 2010, 2, 596-622. [CrossRef]

19. Aridi, T.; Gauthier, M. Chapter 6. Arborescent polymers with a mesoscopic scale. In Complex Macromolecular Architectures: Synthesis, Characterization, and Self-Assembly, 1st ed.; Hadjichristidis, N., Hirao, A., Tezuka, Y., Du Prez, F., Eds.; John Wiley \& Sons: Hoboken, NJ, USA, 2011; pp. 169-194. ISBN 978-047-082-514-3.

20. Moingeon, F.; Wu, Y.; Cadena-Sánchez, L.; Gauthier, M. Synthesis of arborescent styrene homopolymers and copolymers from epoxidized substrates. J. Polym. Sci. A Polym. Chem. 2012, 50, 1819-1826. [CrossRef]

21. Whitton, G.; Gauthier, M. Arborescent polypeptides from $\gamma$-benzyl 1 -glutamic acid. J. Polym. Sci. A Polym. Chem. 2013, 51, 5270-5279. [CrossRef]

22. Aridi, T.; Gauthier, M. Synthesis of arborescent polymers by click grafting. Mater. Res. Soc. Symp. Proc. 2014, 1613, 23-31. [CrossRef]

23. Dockendorff, J.; Gauthier, M. Synthesis of arborescent polystyrene-g-[poly(2-vinylpyridine)-b- polystyrene] core-shell-corona copolymers. J. Polym. Sci. A Polym. Chem. 2014, 52, 1075-1085. [CrossRef]

24. Whitton, G.; Gauthier, M. Arborescent micelles: Dendritic poly $(\gamma$-benzyl 1 -glutamate $)$ cores grafted with hydrophilic chain segments. J. Polym. Sci. A Polym. Chem. 2016, 54, 1197-1209. [CrossRef]

25. Gauthier, M.; Whitton, G. Arborescent unimolecular micelles: Poly( $\gamma$-benzyl L-glutamate) core grafted with a hydrophilic shell by copper(I)-catalyzed azide-alkyne cycloaddition coupling. Polymers $\mathbf{2 0 1 7}, 9,540$. [CrossRef]

26. Gauthier, M.; Aridi, T. Synthesis of arborescent polystyrene by "click" grafting. J. Polym. Sci. A Polym. Chem. 2019, 57, 1730-1740. [CrossRef]

27. Roy, D.; Semsarilar, M.; Guthrie, J.T.; Perrier, S. Cellulose modification by polymer grafting: A review. Chem. Soc. Rev. 2009, 38, 2046-2064. [CrossRef] [PubMed]

28. Wohlhauser, S.; Delepierre, G.; Labet, M.; Morandi, G.; Thielemans, W.; Weder, C.; Zoppe, J.O. Grafting Polymers from Cellulose Nanocrystals: Synthesis, Properties, and Applications. Macromolecules 2018, 51, 6157-6189. [CrossRef]

29. Jenkins, D.W.; Hudson, S.M. Review of vinyl graft copolymerization featuring recent advances toward controlled radical-based reactions and illustrated with chitin/chitosan trunk polymers. Chem. Rev. 2001, 101, 3245-3274. [CrossRef] [PubMed]

30. Thakur, V.K.; Thakur, M.K. Recent Advances in Graft Copolymerization and Applications of Chitosan: A Review. ACS Sustain. Chem. Eng. 2014, 2, 2637-2652. [CrossRef]

31. Kaur, L.; Gupta, G.D. A review on microwave assisted grafting of polymers. Int. J. Pharm. Sci. Res. 2017, 8, 422-426. [CrossRef] 
32. Brodin, M.; Vallejos, M.; Opedal, M.T.; Area, M.C.; Chinga-Carrasco, G. Lignocellulosics as sustainable resources for production of bioplastics-A review. J. Clean. Prod. 2017, 162, 646-664. [CrossRef]

33. Niphadkar, S.; Bagade, P.; Ahmed, S. Bioethanol production: Insight into past, present and future perspectives. Biofuels 2018, 9 , 229-238. [CrossRef]

34. Banerjee, J.; Singh, R.; Vijayaraghavan, R.; MacFarlane, D.; Patti, A.F.; Arora, A. Bioactives from fruit processing wastes: Green approaches to valuable chemicals. Food Chem. 2017, 225, 10-22. [CrossRef]

35. Neuling, U.; Kaltschmitt, M. Review of Biofuel Production-Feedstock, Processes and Markets. J. Oil Palm Res. 2019, 29 , 137-167. [CrossRef]

36. Vega-Hernández, M.Á.; Rosas-Aburto, A.; Vivaldo-Lima, E.; Vázquez-Torres, H.; Cano-Díaz, G.S.; Pérez-Salinas, P.; HernándezLuna, M.G.; Alcaraz-Cienfuegos, J.; Zolotukhin, M.G. Development of polystyrene composites based on blue agave bagasse by in situ RAFT polymerization. J. Appl. Polym. Sci. 2019, 136, 47089. [CrossRef]

37. Farhat, W.; Venditti, R.; Ayoub, A.; Prochazka, F.; Fernández-de-Alba, C.; Mignard, N.; Taha, M.; Becquart, F. Towards thermoplastic hemicellulose: Chemistry and characteristics of poly-( $\varepsilon$-caprolactone) grafting onto hemicellulose backbones. Mater. Des. 2018, 153, 298-307. [CrossRef]

38. Sun, Y.; Ma, Z.; Xu, X.; Liu, X.; Liu, L.; Huang, G.; Liu, L.; Wang, H.; Song, P. Grafting Lignin with Bioderived Polyacrylates for Low-Cost, Ductile, and Fully Biobased Poly(lactic acid) Composites. ACS Sustain. Chem. Eng. 2020, 8, 2267-2276. [CrossRef]

39. Fox, T.G.; Gluckman, M.S.; Gornick, F.; Graham, R.K.; Gratch, S. Graft copolymers from polymers having pendant mercaptan groups. I. Kinetic considerations. J. Polym. Sci. 1959, XXXVII, 397-409. [CrossRef]

40. Zimmerman, J. Molecular weight distributions of vinyl polymers grafted to a solid polymeric substrate by irradiation (theoretical). J. Polym. Sci. 1960, XLIV, 107-116. [CrossRef]

41. Hernández-Ortiz, J.C.; Van Steenberge, P.H.M.; Duchateau, J.N.E.; Toloza, C.; Schreurs, F.; Reyniers, M.-F.; Marin, G.B.; D’hooge, D.R. A two-phase stochastic model to describe mass transport and kinetics during reactive processing of polyolefins. Chem. Eng. J. 2019, 377, 119980. [CrossRef]

42. Gandhi, A.; Verma, S.; Imam, S.S.; Vyas, M. A review on techniques for grafting of natural polymers and their applications. Plant Arch 2019, 19, 972-978.

43. Wei, L.; McDonald, A.G. A Review on Grafting of Biofibers for Biocomposites. Materials 2016, 9, 303. [CrossRef]

44. De Jesús Muñoz Prieto, E.; Rivas, B.; Sánchez, J. Natural polymer grafted with syntethic monomer by microwave for water treatment-A review. Cienc. Desarro. 2012, 4, 219-240. [CrossRef]

45. Barsbay, M.; Güven, O. A short review of radiation-induced raft-mediated graft copolymerization: A powerful combination for modifying the surface properties of polymers in a controlled manner. Radiat. Phys. Chem. 2009, 78, 1054-1059. [CrossRef]

46. Francis, R.; Joy, N.; Aparna, E.P.; Vijayan, R. Polymer Grafted Inorganic Nanoparticles, Preparation, Properties, and Applications: A Review. Polym. Rev. 2014, 54, 268-347. [CrossRef]

47. Garcia-Valdez, O.; Champagne, P.; Cunningham, M.F. Graft modification of natural polysaccharides via reversible deactivation radical polymerization. Prog. Polym. Sci. 2018, 76, 151-173. [CrossRef]

48. Sun, H.; Yang, L.; Thompson, M.P.; Schara, S.; Cao, W.; Choi, W.C.; Hu, Z.; Zang, N.; Tan, W.; Gianneschi, N.C. Recent Advances in Amphiphilic Polymer-Oligonucleotide Nanomaterials via Living/Controlled Polymerization Technologies. Bioconjugate Chem. 2019, 30, 1889-1904. [CrossRef] [PubMed]

49. Chung, T.C. Synthesis of functional polyolefin copolymers with graft and block structures. Prog. Polym. Sci. 2002, $27,39-85$. [CrossRef]

50. Zhou, T.; Zhu, Y.; Li, X.; Liu, X.; Yeung, K.W.K.; Wu, S.; Wang, X.; Cui, Z.; Yang, X.; Chu, P.K. Surface functionalization of biomaterials by radical polymerization. Prog. Mater. Sci. 2016, 83, 191-235. [CrossRef]

51. Ayyavoo, J.; Nguyen, T.P.N.; Jun, B.-M.; Kim, I.-C.; Kwon, Y.N. Protection of polymeric membranes with antifouling surfacing via surface modifications. Colloids Surf. A Physicochem. Eng. Asp. 2016, 506, 190-201. [CrossRef]

52. Weber, C.; Hoogenboom, R.; Schubert, U.S. Temperature responsive bio-compatible polymers based on poly(ethylene oxide) and poly(2-oxazoline)s. Prog. Polym. Sci. 2012, 37, 686-714. [CrossRef]

53. Sun, H.; Choi, W.; Zang, N.; Battistella, C.; Thompson, M.P.; Cao, W.; Zhou, X.; Forman, C.; Gianneschi, N.C. Bioactive Peptide Brush Polymers via Photoinduced Reversible-Deactivation Radical Polymerization. Angew. Chem. Int. Ed. 2019, 58, 17359-17364. [CrossRef]

54. Kumar, R.; Sharma, R.K.; Singh, A.P. Grafted cellulose: A bio-based polymer for durable applications. Polym. Bull. 2018, 75, 2213-2242. [CrossRef]

55. Sharma, S.; Kumar, A. Lignin. Biosynthesis and Transformation for Industrial Applications; Springer Series on Polymer and Composite Materials; Springer Nature: Cham, Switzerland, 2020; pp. 1-252. ISBN 978-303-040-663-9.

56. Mourya, V.K.; Inamdar, N.N. Chitosan-modifications and applications. React. Funct. Polym. 2008, 68, 1013-1051. [CrossRef]

57. Argüelles-Monal, W.M.; Lizardi-Mendoza, J.; Fernández-Quiroz, D.; Recillas-Mota, M.T.; Montiel-Herrera, M. Chitosan Derivatives: Inducing new functionalities with a controlled molecular architecture for innovative materials. Polymers 2018, 10, 342. [CrossRef]

58. Kurita, K. Controlled functionalization of the polysaccharide chitin. Prog. Polym. Sci. 2001, 26, 1921-1971. [CrossRef]

59. Lele, V.V.; Kumari, S.; Niju, H. Syntheses, characterization and applications of graft copolymers of sago starch. Starch 2018, 70, 1700133. [CrossRef] 
60. Radhakrishnan, B.; Ranjan, R.; Brittain, W.J. Surface initiated polymerization from silica nanoparticles. Soft Matter 2006, 2, 386-396. [CrossRef] [PubMed]

61. Foster, J.C.; Radzinsky, S.C.; Matson, J.B. Graft polymer synthesis by RAFT transfer-to. J. Polym. Sci. A Polym. Chem. 2017, 55, 2865-2876. [CrossRef]

62. Bhattacharya, A. Radiation and industrial polymers. Prog. Polym. Sci. 2000, 25, 371-401. [CrossRef]

63. Desmet, T.; Morent, R.; De Geyter, N.; Leys, C.; Schacht, E.; Dubruel, P. Nonthermal plasma technology as a versatile strategy for polymeric biomaterials surface modification: A review. Biomacromolecules 2009, 10, 2351-2378. [CrossRef]

64. Ngo, T.H.A.; Tran, D.T.; Dinh, C.H. Surface photochemical graft polymerization of acrylic acid onto polyamide thin film composite membranes. J. Appl. Polym. Sci. 2017, 134, 44418. [CrossRef]

65. Singh, V.; Tiwari, A.; Tripathi, D.N.; Sanghi, R. Microwave assisted synthesis of guar-g-polyacrylamide. Carbohydr. Polym. 2004, 58, 1-6. [CrossRef]

66. Bhattacharya, A.; Misra, B.N. Grafting: A versatile means to modify polymers technics, factors and applications. Prog. Polym. Sci. 2004, 29, 767-814. [CrossRef]

67. Sosnik, A.; Gotelli, G.; Abraham, G.A. Microwave-assisted polymer synthesis (MAPS) as a tool in biomaterials science: How new and how powerful. Prog. Polym. Sci. 2011, 36, 1050-1078. [CrossRef]

68. Kumar, D.; Pandey, J.; Raj, V.; Kumar, P. A review on the modification of polysaccharide through graft copolymerization for various potential applications. Open Med. Chem. J. 2017, 11, 109-126. [CrossRef]

69. Fan, G.; Zhao, J.; Zhang, Y.; Guo, Z. Grafting modification of kevlar fiber using horseradish peroxidase. Polym. Bull. 2006, 56, 507-515. [CrossRef]

70. Cannatelli, M.D.; Ragauskas, A.J. Conversion of lignin into value-added materials and chemicals via laccase-assisted copolymerization. Appl. Microbiol. Biotechnol. 2016, 100, 8685-8691. [CrossRef] [PubMed]

71. Ran, J.; Wu, L.; Zhang, Z.; Xu, T. Atom transfer radical polymerization (ATRP): A versatile and forceful tool for functional membranes. Prog. Polym. Sci. 2014, 39, 124-144. [CrossRef]

72. Crawford, D.E. Extrusion-back to the future: Using an established technique to reform automated chemical synthesis. Beilstein J. Org. Chem. 2017, 13, 65-75. [CrossRef]

73. Moad, G. The synthesis of polyolefin graft copolymers by reactive extrusion. Prog. Polym. Sci. 1999, 24, 81-142. [CrossRef]

74. Moad, G. Chemical modification of starch by reactive extrusion. Prog. Polym. Sci. 2011, 36, 218-237. [CrossRef]

75. Russell, K.E. Free radical graft polymerization and copolimerization at higher temperatures. Prog. Polym. Sci. 2002, 27, 1007-1038. [CrossRef]

76. Monties, B. Les Polymères Végétaux: Polymères Pariétaux et Alimentaires non Azotés, 1st ed.; Gauthier-Villars: Paris, France, 1980; ISBN 978-204-010-480-1.

77. Casarrubias-Cervantes, R.A. Análisis Fisicoquímico de Procesos de Pretratamiento de Materiales Lignocelulósicos para su Uso en Polímeros Conductores. Bachelor Degree, Facultad de Química-Universidad Nacional Autónoma de México, Ciudad Universitaria, CDMX, 2019, Biblioteca Digital UNAM. Available online: http://132.248.9.195/ptd2019/abril/0788572/Index.html (accessed on 25 May 2020).

78. Koshijima, T.; Muraki, E. Radiation Grafting of Methyl Methacrylate onto Lignin. J. Jpn. Wood Res. Soc. 1964, 10, $110-115$.

79. Koshijima, T.; Muraki, E. Degradation of Lignin-Methyl Metacrylate Graft Copolymer by $\gamma$-Ray Irradiation. J. Jpn. Wood Res. Soc. 1964, 10, 116-119.

80. Koshijima, T.; Timell, T.E. Factors Affecting Number Average Molecular Weights Determination of Hardwood Xylan. J. Jpn. Wood Res. Soc. 1966, 12, 166-172.

81. Koshijima, T.; Muraki, E. Solvent Effects upon Radiation-Induced Graft-copolymerization of Styrene onto Lignin. J. Jpn. Wood Res. Soc. 1966, 12, 139.

82. Koshijima, T. Oxidation of Lignin-Styrene Graft polymer. J. Jpn. Wood Res. Soc. 1966, 12, 114.

83. Koshijima, T.; Muraki, E. Radial Grafting on Lignin (II). Grafting of styrene into Lignin by Initiators. J. Jpn. Wood Res. Soc. 1967, 13, 355-358.

84. Koshijima, T.; Muraki, E.; Naito, K.; Adachi, K. Radical Grafting on Lignin. IV. Semi-Conductive Properties of Lignin-Styrene Graftpolymer. J. Jpn. Wood Res. Soc. 1968, 14, 52-54.

85. Meister, J.J. Modification of Lignin. J. Macromol. Sci. Polymer Rev. 2002, 42, 235-289. [CrossRef]

86. Hon, D.N.S. Chemical Modification of Lignocellulosic Materials, 1st ed.; Marcel Dekker, Inc.: New York, NY, USA, 1996; ISBN 978-082-479-472-9.

87. McDowall, D.J.; Gupta, B.S.; Stannett, V.T. Grafting of vinyl monomers to cellulose by ceric ion initiation. Prog. Polym. Sci. 1984, 10, 1-50. [CrossRef]

88. Bhattacharyya, S.N.; Maldas, D. Graft copolymerization onto cellulosics. Prog. Polym. Sci. 1984, 10, 171-270. [CrossRef]

89. Hon, D.N.S. Graft Copolymerization of Lignocellulosic Fibers; ACS Symposium Series 187; American Chemical Society: Washington, DC, USA, 1982; ISBN 978-084-120-721-9.

90. Mansour, O.Y.; Nagaty, A. Grafting of synthetic polymers to natural polymers by chemical processes. Prog. Polym. Sci. 1985, 11, 91-165. [CrossRef]

91. Feldman, D.; Lacasse, M.; Bernaczuk, L.M. Lignin-polymer sytems and some applications. Prog. Polym. Sci. 1986, 12, 271-299. [CrossRef] 
92. Matyjaszewski, K.; Möller, M. Celluloses and polyoses/hemicelluloses. In Polymer Science: A Comprehensive Reference; Elsevier: Amsterdam, The Netherlands, 2012; ISBN 978-008-087-862-1.

93. Pantelakis, S.; Tserpes, K. Revolutionizing Aircraft Materials and Processes; Springer Nature AG: Cham, Switzerland, 2020; ISBN 978-303-035-346-9.

94. Rol, F.; Belgacem, M.N.; Gandinia, A.; Bras, J. Recent advances in surface-modified cellulose nanofibrils. Prog. Polym. Sci. 2019, 88, 241-264. [CrossRef]

95. Zahran, M.K.; Morsy, M.; Mahmoud, R. Grafting of acrylic monomers onto cotton fabric using an activated cellulose thiocarbonateazobisisobutyronitrile redox system. J. Appl. Polym. Sci. 2003, 91, 1261-1274. [CrossRef]

96. Chauhan, G.S.; Lal, H.; Sharma, R.; Sarwade, B.D. Grafting of a styrene-acrylonitrile binary monomer mixture onto cellulose extracted from pine needles. J. Appl. Polym. Sci. 2001, 83, 2000-2007. [CrossRef]

97. Sabaa, M.W.; Mokhtar, S.M. Chemically induced graft copolymerization of itaconic acid onto cellulose fibers. Polym. Test. 2002, 21, 337-343. [CrossRef]

98. Gupta, K.C.; Sahoo, S. Grafting of N,N'-methylenebisacrylamide onto cellulose using Co(III)-acetylacetonate complex in aqueous medium. J. Appl. Polym. Sci. 2000, 76, 906-912. [CrossRef]

99. Gupta, K.C.; Sahoo, S. Co(III) acetylacetonate-complex-initiated grafting of N-vinyl pyrrolidone on cellulose in aqueous media. J. Appl. Polym. Sci. 2001, 81, 2286-2296. [CrossRef]

100. Gupta, K.C.; Khandekar, K. Temperature-Responsive Cellulose by Ceric(IV) Ion-Initiated Graft Copolymerization of NIsopropylacrylamide. Biomacromolecules 2003, 4, 758-765. [CrossRef]

101. Gupta, K.C.; Khandekar, K. Graft copolymerization of acrylamide-methylacrylate comonomers onto cellulose using ceric ammonium nitrate. J. Appl. Polym. Sci. 2002, 86, 2631-2642. [CrossRef]

102. Gupta, K.C.; Khandekar, K. Graft copolymerization of acrylamide onto cellulose in presence of comonomer using ceric ammonium nitrate as initiator. J. Appl. Polym. Sci. 2006, 101, 2546-2558. [CrossRef]

103. Gupta, K.C.; Sahoo, S. Graft Copolymerization of Acrylonitrile and Ethyl Methacrylate Comonomers on Cellulose Using Ceric Ions. Biomacromolecules 2001, 2, 239-247. [CrossRef]

104. Gupta, K.C.; Sahoo, S.; Khandekar, K. Graft Copolymerization of Ethyl Acrylate onto Cellulose Using Ceric Ammonium Nitrate as Initiator in Aqueous Medium. Biomacromolecules 2002, 3, 1087-1094. [CrossRef] [PubMed]

105. Gupta, K.C.; Khandekar, K. Ceric(IV) ion-induced graft copolymerization of acrylamide and ethyl acrylate onto cellulose. Polym. Int. 2005, 55, 139-150. [CrossRef]

106. Toledano-Thompson, T.; Loría-Bastarrachea, M.I.; Aguilar-Vega, M.J. Characterization of henequen cellulose microfibers treated with an epoxide and grafted with poly(acrylic acid). Carbohydr. Polym. 2005, 62, 67-73. [CrossRef]

107. Mansour, O.Y.; Nagieb, Z.A.; Basta, A.H. Graft polymerization of some vinyl monomers onto alkali-treated cellulose. J. Appl. Polym. Sci. 1991, 43, 1147-1158. [CrossRef]

108. Semsarilar, M.; Ladmiral, V.; Perrier, S. Synthesis of a cellulose supported chain transfer agent and its application to RAFT polymerization. J. Polym. Sci. A Polym. Chem. 2010, 48, 4361-4365. [CrossRef]

109. Cankaya, N.; Temüz, M. Characterization and monomer reactivity ratios of grafted cellulose with n-(4-nitrophenyl)acrylamide and methyl methacrylate by atom transfer radical polymerization. Cell. Chem. Technol. 2012, 46, 551-558.

110. Thakur, V.K.; Thakur, M.K.; Gupta, R.K. Rapid synthesis of graft copolymers from natural cellulose fibers. Carbohydr. Polym. 2013, 98, 820-828. [CrossRef]

111. Routray, C.; Tosh, B. Graft copolymerization of methyl methacrylate (mma) onto cellulose acetate in homogeneous medium: Effect of solvent, initiator and homopolymer inhibitor. Cell. Chem. Technol. 2013, 47, 171-190.

112. Cankaya, N.; Temüz, M.M. Monomer reactivity ratios of cellulose grafted with N-cyclohexylacrylamide and methyl methacrylate by atom transfer radical polymerization. Cell. Chem. Technol. 2014, 48, 209-215.

113. Popescu, O.; Dunca, S.; Grigoriu, A. Antibacterial action of silver applied on cellulose fibers grafted with monochlorotriazinyl- $\beta$ cyclodextrin. Cell. Chem. Technol. 2013, 47, 247-255.

114. Popescu, O.; Grigoriu, A.; Diaconescu, R.M.; Vasluianu, E. Optimization of the cellulosic materials functionalization with monochlorotriazinyl- $\beta$-cyclodextrin in basic medium. Ind. Textilá 2012, 63, 68-75.

115. Sun, Z.; Chen, F. Homogeneous grafting copolymerization of methylmethacrylate onto cellulose using ammonium persulfate. Cell. Chem. Technol. 2014, 48, 217-223.

116. Dai, L.; Shen, Y.; Li, D.; Xiao, S.; He, J. Cellulose-graft-poly(l-lactide) as a degradable drugdelivery system: Synthesis, degradation and drug release. Cell. Chem. Technol. 2014, 48, 237-245.

117. Xiaoming, S.; Songlin, W.; Shanshan, G.; Fushan, C.; Fusheng, L. Study on grafting copolymerization of methyl methacrylate onto cellulose under heterogeneous conditions. Cell. Chem. Technol. 2016, 50, 65-70.

118. Yin, Y.; Jiang, T.X.; Wang, H.; Gao, W. Modification of cellulose nanocrystal via SI-ATRP of styrene and themechanism of its reinforcement of polymethylmethacrylate. Carbohydr. Polym. 2016, 142, 206-212. [CrossRef] [PubMed]

119. Paula, E.L.; Roig, F.; Mas, A.; Habas, J.P.; Mano, V.; Vargas Pereira, F.; Robin, J.J. Effect of surface-grafted cellulose nanocrystals on the thermal and mechanical properties of PLLA based nanocomposites. Eur. Polym. J. 2016, 84, 173-187. [CrossRef]

120. Zhao, C.; Li, J.; He, B.; Zhao, L. Fabrication of hydrophobic biocomposite by combining cellulosic fibers with polyhydroxyalkanoate. Cellulose 2017, 24, 2265-2274. [CrossRef] 
121. Badawy, S.M. Functional cellulosic filter papers prepared by radiation-induced graft copolymerization for chelation of rare earth elements. Cell. Chem. Technol. 2017, 51, 551-558.

122. Çankaya, N.; Temüz, M.M.; Yakuphanoglu, F. Grafting of some monomers onto cellulose by atom transfer radical polymerization. Electrical conductivity and thermal properties of resulting copolymers. Cell. Chem. Technol. 2018, 52, $19-26$.

123. Müssig, J.; Kelch, M.; Gebert, B.; Hohe, J.; Luke, M.; Bahners, T. Improvement of the fatigue behaviour of cellulose/polyolefin composites using photo-chemical fibre surface modification bio-inspired by natural role models. Cellulose 2020, 27, 5815-5827. [CrossRef]

124. Chen, Y.; Yu, Z.; Han, Y.; Yang, S.; Fan, D.; Li, G.; Wang, S. Combination of water soluble chemical grafting and gradient freezing to fabricate elasticity enhanced and anisotropic nanocellulose aerogels. Appl. Nanosci. 2020, 10, 411-419. [CrossRef]

125. Eraghi Kazzaz, A.; Hosseinpour Feizi, Z.; Fatehi, P. Grafting strategies for hydroxy groups of lignin for producing materials. Green Chem. 2019, 21, 5714-5752. [CrossRef]

126. Abe, A.; Dusek, K.; Kobayashi, S. Biopolymers. Lignin, Proteins, Bioactive Nanocomposites, 1st ed.; Springer: Heidelberg/Berlin, Germany, 2010; Volume 232, ISBN 978-364-213-630-6.

127. Huang, J.; Fu, S.; Gan, L. Lignin Chemistry and Applications, 1st ed.; Elsevier: Amsterdam, The Netherlands, 2019; ISBN 978-012813-963-9.

128. Marton, J. Lignin. Structure and Reactions, 1st ed.; Advances in Chemistry Series 59; American Chemical Society: Washington, DC, USA, 1966; ISBN 978-084-122-239-7.

129. Glasser, W.G.; Sarkanen, S. Lignin. Properties and Materials, 1st ed.; ACS Symposium Series 397; American Chemical Society: Washington, DC, USA, 1989; ISBN 978-084-121-248-0.

130. Lewis, N.G.; Sarkanen, S. Lignin and Lignan Biosynthesis; ACS Symposium Series 697; American Chemical Society: Washington, DC, USA, 1998; ISBN 084-123-566-X.

131. Katahira, R.; Elder, T.J.; Beckham, G.T. Chapter 1 A brief introduction to lignin structure. In Lignin Valorization. Emerging Approaches, 1st ed.; Beckham, G.T., Ed.; The Royal Society of Chemistry: Croydon, London, UK, 2018; pp. 1-20, ISBN 978-178-801035-1.

132. Laurichesse, S.; Avérous, L. Chemical modification of lignins: Towards biobased polymers. Prog. Polym. Sci. 2014, 39, 1266-1290. [CrossRef]

133. Figueiredo, P.; Lintinen, K.; Hirvonen, J.T.; Kostiainen, M.A.; Santos, H.A. Properties and chemical modifications of lignin: Towards lignin-based nanomaterials for biomedical applications. Prog. Mater. Sci. 2018, 93, 233-269. [CrossRef]

134. Xu, Y.; Yuan, L.; Wang, Z.; Wilbon, P.A.; Wang, C.; Chu, F.; Tang, C. Lignin and soy oil-derived polymeric biocomposites by "grafting from" RAFT polymerization. Green Chem. 2016, 18, 4974-4981. [CrossRef]

135. Yu, Y.; Fu, S.; Song, P.; Lou, X.; Jin, Y.; Lu, F.; Wu, Q.; Ye, J. Functionalized lignin by grafting phosphorus-nitrogen improves the thermal stability and flame retardancy of polypropylene. Polym. Degrad. Stabil. 2012, 97, 541-546. [CrossRef]

136. Prieur, B.; Meub, M.; Wittermann, M.; Klein, R.; Bellayer, S.; Fontaine, G.; Bourbigot, S. Phosphorylation of lignin: Characterization and investigation of the thermal decomposition. RSC Adv. 2017, 7, 16866-16877. [CrossRef]

137. Prieur, B.; Meub, M.; Wittemann, M.; Klein, R.; Bellayer, S.; Fontaine, G.; Bourbigot, S. Phosphorylation of lignin to flame retard acrylonitrile butadiene styrene (ABS). Polym. Degrad. Stabil. 2016, 127, 32-43. [CrossRef]

138. Liu, H.; Chung, H. Lignin-Based Polymers via Graft Copolymerization. J. Polym. Sci. A Polym. Chem. 2017, 55, 3515-3528. [CrossRef]

139. Gupta, C.; Washburn, N.R. Polymer-grafted lignin surfactants prepared via Reversible Addition-Fragmentation Chain-Transfer polymerization. Langmuir 2014, 30, 9303-9312. [CrossRef]

140. Ganewatta, M.S.; Lokupitiya, H.N.; Tang, C. Lignin biopolymers in the age of controlled polymerization. Polymers 2019, 11, 1176. [CrossRef]

141. Kim, Y.S.; Kadla, J.F. Preparation of a thermoresponsive lignin-based biomaterial through atom transfer radical polymerization. Biomacromolecules 2010, 11, 981-988. [CrossRef] [PubMed]

142. Wang, J.; Yao, J.; Korich, K.; Li, S.; Ma, S.; Ploehn, H.J.; Iovine, P.M.; Wang, C.; Chu, F.; Tang, C. Combining renewable gum rosin and lignin: Towards hydrophobic polymer composites by controlled polymerization. J. Polym. Sci. A Polym. Chem. 2011, 49, 3728-3738. [CrossRef]

143. Diao, B.; Zhang, Z.; Zhu, J.; Li, J. Biomass-based thermogelling copolymers consisting of lignin and grafted poly (Nisopropylacrylamide), poly (ethylene glycol), and poly (propylene glycol). RSC Adv. 2014, 4, 42996-43003. [CrossRef]

144. Yu, J.; Wang, J.; Wang, C.; Liu, Y.; Xu, Y.; Tang, C.; Chu, F. UV-Absorbent Lignin-Based Multi-Arm Star Thermoplastic Elastomers. Macromol. Rapid Commun. 2015, 36, 398-404. [CrossRef]

145. Gao, G.; Dallmeyer, J.I.; Kadla, J.F. Synthesis of lignin nanofibers with ionic-responsive shells: Water-expandable lignin-based nanofibrous mats. Biomacromolecules 2012, 13, 3602-3610. [CrossRef] [PubMed]

146. Hilburg, S.L.; Elder, A.N.; Chung, H.; Ferebee, R.L.; Bockstaller, M.R.; Washburn, N.R. A universal route towards thermoplastic lignin composites with improved mechanical properties. Polymer 2014, 55, 995-1003. [CrossRef]

147. Shah, T.; Gupta, C.; Ferebee, R.L.; Bockstaller, M.R.; Washburn, N.R. Extraordinary toughening and strengthening effect in polymer nanocomposites using lignin-based fillers synthesized by ATRP. Polymer 2015, 72, 406-412. [CrossRef]

148. Kai, D.; Low, Z.W.; Liow, S.S.; Abdul Karim, A.; Ye, H.; Jin, G.; Li, K.; Loh, X.J. Development of lignin supramolecular hydrogels with mechanically responsive and self-healing properties. ACS Sustain. Chem. Eng. 2015, 3, 2160-2169. [CrossRef] 
149. Li, H.; Pang, Z.; Gao, P.; Wang, L. Fe (III)-catalyzed grafting copolymerization of lignin with styrene and methyl methacrylate through AGET ATRP using triphenyl phosphine as a ligand. RSC Adv. 2015, 5, 54387-54394. [CrossRef]

150. Liu, X.; Yin, H.; Zhang, Z.; Diao, B.; Li, G. Functionalization of lignin through ATRP grafting of poly(2-dimethylaminoethyl methacrylate) for gene delivery. Colloids Surf. B Biointerfaces 2015, 125, 230-237. [CrossRef] [PubMed]

151. Silmore, K.S.; Gupta, C.; Washburn, N.R. Tunable Pickering emulsions with polymer-grafted lignin nanoparticles (PGLNs). J. Colloid Interface Sci. 2016, 466, 91-100. [CrossRef] [PubMed]

152. Gupta, C.; Nadelman, E.; Washburn, N.R.; Kurtis, K.E. Lignopolymer Superplasticizers for Low-CO2 Cements. ACS Sustain. Chem. Eng. 2017, 5, 4041-4049. [CrossRef]

153. Gupta, C.; Sverdlove, M.J.; Washburn, N.R. Molecular architecture requirements for polymer-grafted lignin superplasticizers. Soft Matter 2015, 11, 2691-2699. [CrossRef]

154. Liu, Z.; Lu, X.; Xie, J.; Feng, B.; Han, Q. Synthesis of a novel tunable lignin-based star copolymer and its flocculation performance in the treatment of kaolin suspension. Sep. Purif. Technol. 2019, 210, 355-363. [CrossRef]

155. Nemoto, T.; Konishi, G.-I.; Tojo, Y.; An, Y.C.; Funaoka, M. Functionalization of lignin: Synthesis of lignophenol-graft-poly (2-ethyl-2-oxazoline) and its application to polymer blends with commodity polymer. J. Appl. Polym. Sci. 2012, 123, 2636-2642. [CrossRef]

156. Mahata, D.; Jana, M.; Jana, A.; Mukherjee, A.; Mondal, N.; Saha, T.; Sen, S.; Nando, G.B.; Mukhopadhyay, C.K.; Chakraborty, R.; et al. Lignin-graft-polyoxazoline conjugated triazole a novel anti-infective ointment to control persistent inflammation. Sci. Rep. 2017, 7, 46412. [CrossRef] [PubMed]

157. Chung, Y.L.; Olsson, J.V.; Li, R.J.; Frank, C.W.; Waymouth, R.M.; Billington, S.L.; Sattely, E.S. A renewable lignin-lactide copolymer and application in biobased composite. ACS Sustain. Chem. Eng. 2013, 1, 1231-1238. [CrossRef]

158. Liu, X.; Zong, E.; Jiang, J.; Fu, S.; Wang, J.; Xu, B.; Li, W.; Lin, X.; Xu, Y.; Wang, C.; et al. Preparation and characterization of Lignin-graft-poly (E-caprolactone) copolymers based on lignocellulosic butanol residue. Int. J. Biol. Macromol. 2015, 81, 521-529. [CrossRef]

159. Sun, Y.; Yang, L.; Lu, X.; He, C. Biodegradable and renewable poly (lactide)-lignin composites: Synthesis, interface and toughening mechanism. J. Mater. Chem. A 2015, 3, 3699-3709. [CrossRef]

160. Kai, D.; Zhang, K.; Liow, S.S.; Loh, X.J. New dual functional phb-grafted lignin copolymer: Synthesis, mechanical properties, and biocompatibility studies. ACS Appl. Bio Mater. 2018, 2, 127-134. [CrossRef]

161. Pérez-Camargo, R.A.; Saenz, G.; Laurichesse, S.; Casas, M.T.; Puiggalí, J.; Avérous, L.; Müller, A.J. Nucleation crystallization, and thermal fractionation of poly ( $\varepsilon$-caprolactone)-grafted-lignin: Effect of grafted chains length and lignin content. J. Polym. Sci. Part B Polym. Phys. 2015, 53, 1736-1750. [CrossRef]

162. Laurichesse, S.; Avérous, L. Synthesis, thermal properties, rheological and mechanical behaviors of lignins-grafted-poly( $\varepsilon$ caprolactone). Polymer 2013, 54, 3882-3890. [CrossRef]

163. Schmidt, B.V.K.J.; Molinari, V.; Esposito, D.; Tauer, K.; Antonietti, M. Lignin-based polymeric surfactants for emulsion polymerization. Polymer 2017, 112, 418-426. [CrossRef]

164. Tapdiqov, S.Z. A drug-loaded gel based on graft radical co-polymerization of n-vinylpyrrolidone and 4-vinylpyridine with chitosan. Cell. Chem. Technol. 2020, 54, 429-438. [CrossRef]

165. Cheaburu-Yilmaz, C.N. On the development of chitosan-graft-poly(n-isopropylacrylamide) by raft polymerization technique. Cell. Chem. Technol. 2020, 54, 1-10. [CrossRef]

166. Kadokawa, J.-I. Preparation and Grafting Functionalization of Self-Assembled Chitin Nanofiber Film. Coatings 2016, 6, 27. [CrossRef]

167. Mahmoud, G.A.; Sayed, A.; Thabit, M.; Safwat, G. Chitosan biopolymer based nanocomposite hydrogels for removal of methylene blue dye. SN Appl. Sci. 2020, 2, 968. [CrossRef]

168. Xu, Y.; Liu, B.; Zou, L.; Sun, C.; Li, W. Preparation and characterization of PLLA/chitosan-graft-poly( $(\varepsilon$-caprolactone) (CS-g-PCL) composite fibrous mats: The microstructure, performance and proliferation assessment. Int. J. Biol. Macromol. 2020, 162, 320-332. [CrossRef] [PubMed]

169. Kadokawa, J.-I. Fabrication of nanostructured a nd microstructured chitin materials through gelation with suitable dispersion media. RSC Adv. 2015, 5, 12736-12746. [CrossRef]

170. Stefan, J.; Lorkowska-Zawicka, B.; Kaminski, K.; Szczubialka, K.; Nowakowska, M.; Korbut, R. The current view on biological potency of cationically modified chitosan. J. Physiol. Pharmacol. 2014, 65, 341-347. [PubMed]

171. Jiang, T.; Deng, M.; James, R.; Nair, L.S.; Laurencin, C.T. Micro- and nanofabrication of chitosan structures for regenerative engineering. Acta Biomater. 2014, 10, 1632-1645. [CrossRef]

172. Lai, G.-J.; Shalumon, K.T.; Chen, S.-H.; Chen, J.P. Composite chitosan/silk fibroin nanofibers for modulation of osteogenic differentiation and proliferation of human mesenchymal stem cells. Carbohydr. Polym. 2014, 111, 288-297. [CrossRef]

173. Jayakumar, R.; Menon, D.; Manzoor, K.; Nair, S.V.; Tamura, H. Biomedical applications of chitin and chitosan based nanomaterialsA short review. Carbohydr. Polym. 2010, 82, 227-232. [CrossRef]

174. Deng, Z.; Wang, T.; Chen, X.; Liu, Y. Applications of chitosan based biomaterials: A focus on dependent antimicrobial properties. Mar. Life Sci. Technol. 2020, 2, 398-413. [CrossRef]

175. Wen, J.; Li, Y.; Wang, L.; Chen, X.; Cao, Q.; He, N. Carbon Dioxide Smart Materials Based on Chitosan. Prog. Chem. 2020, 32, 417-422. (In Chinese) 
176. Sashiwa, H.; Aiba, S.-I. Chemically modified chitin and chitosan as biomaterials. Prog. Polym. Sci. 2004, 29, 887-908. [CrossRef]

177. Crini, G.; Badot, P.-M. Application of chitosan, a natural aminopolysaccharide, for dye removal from aqueous solutions by adsorption processes using batch studies: A review of recent literature. Prog. Polym. Sci. 2008, 33, 399-447. [CrossRef]

178. Mittal, H.; Ray, S.S.; Kaith, B.S.; Bhatia, J.K.; Sharma, S.J.; Alhassan, S.M. Recent progress in the structural modification of chitosan for applications in diversified biomedical fields. Eur. Polym. J. 2018, 109, 402-434. [CrossRef]

179. Iyer, B.V.S.; Yashin, V.V.; Hamer, M.J.; Kowalewski, T.; Matyjaszewski, K.; Balazsa, A.C. Ductility, toughness and strain recovery in self-healing dualcross-linked nanoparticle networks studied by computer simulations. Prog. Polym. Sci. 2015, 40, 121-137. [CrossRef]

180. Derry, M.J.; Fielding, L.A.; Armes, S.P. Polymerization-induced self-assembly of block copolymer nanoparticles via RAFT non-aqueous dispersion polymerization. Prog. Polym. Sci. 2016, 52, 1-18. [CrossRef]

181. Bednarek, M. Branched aliphatic polyesters by ring-opening (co)polymerization. Prog. Polym. Sci. 2016, 58, 27-58. [CrossRef]

182. Yildirim, I.; Weber, C.; Schubert, U.S. Old meets new: Combination of PLA and RDRP to obtain sophisticated macromolecular architectures. Prog. Polym. Sci. 2018, 76, 111-150. [CrossRef]

183. Wang, W.; Lu, W.; Goodwin, A.; Wang, H.; Yin, P.; Kang, N.-G.; Hong, K.; Mays, J.W. Recent advances in thermoplastic elastomers from living polymerizations: Macromolecular architectures and supramolecular chemistry. Prog. Polym. Sci. 2019, 95, 1-31. [CrossRef]

184. Mocny, P.; Klok, H.-A. Complex polymer topologies and polymer-nanoparticle hybrid films prepared via surface-initiated controlled radical polymerization. Prog. Polym. Sci. 2020, 100, 101185. [CrossRef]

185. Vivaldo-Lima, E.; Jaramillo-Soto, G.; Penlidis, A. Nitroxide-mediated polymerization (NMP). In Encyclopedia of Polymer Science and Technology, 1st ed.; John Wiley \& Sons: New York, NY, USA, 2016; pp. 1-48. ISBN 978-047-144-026-0.

186. Olivier, A.; Meyer, F.; Raquez, J.-M.; Damman, P.; Dubois, P. Surface-initiated controlled polymerization as a convenient method for designing functional polymer brushes: From self-assembled monolayers to patterned surfaces. Prog. Polym. Sci. 2012, 37, 157-181. [CrossRef]

187. Radzevicius, P.; Krivorotova, T.; Makuska, R. Synthesis by one-pot RAFT polymerization and properties of amphiphilic pentablock copolymers with repeating blocks of poly(2-hydroxyethyl methacrylate) and poly(butyl methacrylate). Eur. Polym. J. 2017, 87, 69-83. [CrossRef]

188. Chmielarz, P.; Fantin, M.; Park, S.; Isse, A.A.; Gennaro, A.; Magenau, A.J.; Sobkowiak, A.; Matyjaszewski, K. Electrochemically mediated atom transfer radical polymerization (eATRP). Prog. Polym. Sci. 2017, 69, 47-78. [CrossRef]

189. Maharana, T.; Pattanaik, S.; Routaray, A.; Nath, N.; Sutar, A.K. Synthesis and characterization of poly(lactic acid) based graft copolymers. React. Funct. Polym. 2015, 93, 47-67. [CrossRef]

190. Mehta, A.; Pandey, J.P.; Sen, G. Synthesis of Diallyl dimethyl ammonium chloride grafted polyvinyl pyrrolidone (PVP-gDADMAC) and its applications. Mater. Sci. Eng. B Solid State Mater. Adv. Technol. 2021, 263, 114750. [CrossRef]

191. El-Sayed, N.; Awad, H.; El-Sayed, G.M.; Nagieb, Z.A.; Kamel, S. Synthesis and characterization of biocompatible hydrogel based on hydroxyethyl cellulose-g-poly(hydroxyethyl methacrylate). Polym. Bull. 2020, 77, 6333-6347. [CrossRef]

192. Mahdavi, H.; Mazinani, N.; Heidari, A.A. Poly(vinylidene fluoride) (PVDF)/PVDF-g-polyvinylpyrrolidone (PVP)/TiO2 mixed matrix nanofiltration membranes: Preparation and characterization. Polym. Int. 2020, 69, 1187-1195. [CrossRef]

193. Oliveira, T.S.; Brazil, T.R.; Guerrini, L.M.; Rezende, M.C.; Oliveira, M.P. Synthesis and characterization of poly (acrylonitrile-glignin) by semi-batch solution polymerization and evaluation of their potential application as carbon materials. J. Polym. Res. 2020, 27, 340. [CrossRef]

194. Zhang, M.; Yang, P.; Lan, G.; Liu, Y.; Cai, Q.; Xi, J. High crosslinked sodium carboxyl methylstarch-g-poly (acrylic acid-coacrylamide) resin for heavy metal adsorption: Its characteristics and mechanisms. Environ. Sci. Pollut. Res. 2020, 27, 38617-38630. [CrossRef]

195. Wang, L.; Zhang, X.; Xu, J.; Wang, Q.; Fan, X. Synthesis of partly debranched starch-g-poly(2-acryloyloxyethyl trimethyl ammonium chloride) catalyzed by horseradish peroxidase and the effect on adhesion to polyester/cotton yarn. Process Biochem. 2020, 97, 176-182. [CrossRef]

196. Xu, R.-M.; Yang, T.T.; Vidovic, E.; Jia, R.-N.; Zhang, J.-M.; Mi, Q.-Y.; Zhang, J. Cellulose Acetate Thermoplastics with High Modulus, Dimensional Stability and Anti-migration Properties by Using CA-g-PLA as Macromolecular Plasticizer. Chin. J. Polym. Sci. 2020, 38, 1141-1148. [CrossRef]

197. Wu, Q.; Tiraferri, A.; Li, T.; Xie, W.; Chang, H.; Bai, Y.; Liu, B. Superwettable PVDF/PVDF-g-PEGMA Ultrafiltration Membranes. ACS Omega 2020, 5, 23450-23459. [CrossRef] [PubMed]

198. Peighambardoust, S.J.; Aghamohammadi-Bavil, O.; Foroutan, R.; Arsalani, N. Removal of malachite green using carboxymethyl cellulose-g-polyacrylamide/montmorillonite nanocomposite hydrogel. Int. J. Biol. Macromol. 2020, 159, 1122-1131. [CrossRef]

199. Gürsel, U.; Taran, S.; Gökçen, M.; Ari, Y.; Alli, A. Ultraviolet illumination responsivity of the Au/n-Si diodes with and without poly (linolenic acid)-g-poly (caprolactone)-g-poly (t-butyl acrylate) interfacial layer. Surf. Rev. Lett. 2020, 27, 1950207. [CrossRef]

200. Song, P.; Guo, R.; Ma, W.; Wang, L.; Ma, F.; Wang, R. Synthesis of CO2-based polycarbonate-g-polystyrene copolymers via NMRP. Chem. Commun. 2020, 56, 9493-9496. [CrossRef] [PubMed]

201. Klimovica, K.; Pan, S.; Lin, T.-W.; Peng, X.; Ellison, C.J.; LaPointe, A.M.; Bates, F.S.; Coates, G.W. Compatibilization of iPP/HDPE Blends with PE-g-iPP Graft Copolymers. ACS Macro. Lett. 2020, 9, 1161-1166. [CrossRef] 
202. Li, W.; Yu, Z.; Wu, Y.; Liu, Q. Preparation, characterization of feather protein-g-poly(sodium allyl sulfonate) and its application as a low-temperature adhesive to cotton and viscose fibers for warp sizing. Eur. Polym. J. 2020, 136, 109945. [CrossRef]

203. Czarnecka, E.; Nowaczyk, J. Semi-Natural superabsorbents based on Starch-g-poly(acrylic acid): Modification, synthesis and application. Polymers 2020, 12, 1794. [CrossRef]

204. Bhosale, R.R.; Gangadharappa, H.V.; Osmani, R.A.M.; Gowda, D.V. Design and development of polymethylmethacrylate-grafted gellan gum (PMMA-g-GG)-based pH-sensitive novel drug delivery system for antidiabetic therapy. Drug Deliv. and Transl. Res. 2020, 10, 1002-1018. [CrossRef] [PubMed]

205. Patel, R.; Patel, M.; Sung, J.-S.; Kim, J.H. Preparation and characterization of bioinert amphiphilic P(VDF-co-CTFE)-g-POEM graft copolymer. Polym. Plast. Technol. Mater. 2020, 59, 1077-1087. [CrossRef]

206. Li, W.; Wu, Y.; Wu, J.; Ni, Q. Preparation, characterization of poly(acrylic acid)-g-feather protein-g-poly(methyl acrylate) and application in improving adhesion of protein to PLA fibers for sizing. React. Funct. Polym. 2020, 152, 104607. [CrossRef]

207. Deng, J.-R.; Zhao, C.-L.; Wu, Y.-X. Antibacterial and pH-responsive Quaternized Hydroxypropyl Cellulose-g-Poly(THF-coepichlorohydrin) Graft Copolymer: Synthesis, Characterization and Properties. Chin. J. Polym. Sci. 2020, 38, 704-714. [CrossRef]

208. Cuggino, J.C.; Ambrosioni, F.E.; Picchio, M.L.; Nicola, M.; Jiménez Kairuz, A.F.; Gatti, G.; Minari, R.J.; Calderon, M.; Alvarez Igarzabal, C.I.; Gugliotta, L.M. Thermally self-assembled biodegradable poly(casein-g-N-isopropylacrylamide) unimers and their application in drug delivery for cancer therapy. Int. J. Biol. Macromol. 2020, 154, 446-455. [CrossRef] [PubMed]

209. Kenawy, E.R.; Seggiani, M.; Cinelli, P.; Elnaby, H.M.H.; Azaam, M.M. Swelling capacity of sugarcane bagasse-g-poly(acrylamide)/ attapulgite superabsorbent composites and their application as slow release fertilizer. Eur. Polym. J. 2020, 133, 109769. [CrossRef]

210. Jiang, P.; Ji, H.; Li, G.; Chen, S.; Lv, L. Structure formation in pH-sensitive micro porous membrane from well-defined ethyl cellulose-g-PDEAEMA via non-solvent-induced phase separation process. J. Macromol. Sci. Pure Appl. Chem. 2020, 57, 461-471. [CrossRef]

211. Huang, Q.; Xu, Z.; Cai, C.; Lin, J. Micelles with a Loose Core Self-Assembled from Coil-g-Rod Graft Copolymers Displaying High Drug Loading Capacity. Macromol. Chem. Phys. 2020, 221, 2000121. [CrossRef]

212. Wang, Z.; Wu, L.; Zhou, D.; Ji, P.; Zhou, X.; Zhang, Y.; He, P. Synthesis and Water Absorbing Properties of KGM-g-P(AA-AM(DMAEA-EB)) via Grafting Polymerization Method. Polym. Sci. Ser. B 2020, 62, 238-244. [CrossRef]

213. Erdoğan, M.K.; Akdemir, Ö.; Hamitbeyli, A.; Karakışla, M. Preparation of hydrophilic woven fabrics: Surface modification of poly(ethylene terephthalate) by grafting of poly(vinyl alcohol) and poly(vinyl alcohol)-g-(N-vinyl-2-pyrrolidone). J. Appl. Polym. Sci. 2020, 137, 48584. [CrossRef]

214. Worzakowska, M. The preparation, physicochemical and thermal properties of the high moisture, solvent and chemical resistant starch-g-poly(geranyl methacrylate) copolymers. J. Thermal. Anal. Calorim. 2020, 140, 189-198. [CrossRef]

215. Delorme, V.; Lichon, L.; Mahindad, H.; Hunger, S.; Laroui, N.; Daurat, M.; Godefroy, A.; Coudane, J.; Gary-Bobo, M.; Van Den Berghe, H. Reverse poly( $\varepsilon$-caprolactone)-g-dextran graft copolymers. Nano-carriers for intracellular uptake of anticancer drugs. Carbohydr. Polym. 2020, 232, 115764. [CrossRef] [PubMed]

216. Kang, D.A.; Kim, K.; Karade, S.S.; Kim, H.; Kim, J.H. High-performance solid-state bendable supercapacitors based on PEGBEMg-PAEMA graft copolymer electrolyte. Chem. Eng. J. 2020, 384, 123308. [CrossRef]

217. Tian, B.; Cai, Y.; Zhang, X.; Fan, H.; Li, B.-G. Design of Well-Defined Polyethylene-g-poly-methyltrifluorosiloxane Graft Copolymers via Direct Copolymerization of Ethylene with Polyfluorosiloxane Macromonomers. Ind. Eng. Chem. Res. 2020, 59, $4557-4567$. [CrossRef]

218. Öztürk, T.; Meyvac1, E.; Arslan, T. Synthesis and characterization of poly(vinyl chloride-g- $\varepsilon$-caprolactone) brush type graft copolymers by ring-opening polymerization and "click" chemistry. J. Macromol. Sci. Pure Appl. Chem. 2020, 57, 171-180. [CrossRef]

219. Zha, X.; Sadi, M.S.; Yang, Y.; Luo, T.; Huang, N. Adhesion of cornstarch-g-poly (2-hydroxyethyl acrylate) to cotton fibers in sizing. J. Adhes. Sci. Technol. 2020, 34, 461-479. [CrossRef]

220. Nicolas, C.; Zhang, W.; Choppé, E.; Fontaine, L.; Montembault, V. Polynorbornene-g-poly(ethylene oxide) Through the Combination of ROMP and Nitroxide Radical Coupling Reactions. J. Polym. Sci. 2020, 58, 645-653. [CrossRef]

221. Ilhan, E.; Karahaliloglu, Z.; Kilicay, E.; Hazer, B.; Denkbas, E.B. Potent bioactive bone cements impregnated with polystyrene-gsoybean oil-AgNPs for advanced bone tissue applications. Mater. Technol. 2020, 35, 179-194. [CrossRef]

222. Chen, Y.; Li, Q.; Li, Y.; Zhang, Q.; Huang, J.; Wu, Q.; Wang, S. Fabrication of cellulose nanocrystal-g-poly(acrylic acid-coacrylamide) aerogels for efficient $\mathrm{Pb}$ (II) removal. Polymers 2020, 12, 333. [CrossRef]

223. Mo, X.-Z.; Wei, F.-X.; Tan, D.-F.; Pang, J.-Y.; Lan, C.-B. The compatibilization of PLA-g-TPU graft copolymer on polylactide/thermoplastic polyurethane blends. J. Polym. Res. 2020, 27, 33. [CrossRef]

224. Guleria, A.; Kumari, G.; Lima, E.C. Cellulose-g-poly-(acrylamide-co-acrylic acid) polymeric bioadsorbent for the removal of toxic inorganic pollutants from wastewaters. Carbohydr. Polym. 2020, 228, 115396. [CrossRef] [PubMed]

225. He, M.; Li, T.; Hu, M.; Chen, C.; Liu, B.; Crittenden, J.; Chu, L.-Y.; Ng, H.Y. Performance improvement for thin-film composite nanofiltration membranes prepared on PSf/PSf-g-PEG blended substrates. Sep. Purif. Technol. 2020, 230, 115855. [CrossRef]

226. Savaş, B.; Öztürk, T. Synthesis and characterization of poly(vinyl chloride-g-methyl methacrylate) graft copolymer by redox polymerization and $\mathrm{Cu}$ catalyzed azide-alkyne cycloaddition reaction. J. Macromol. Sci. Pure Appl. Chem. 2020, 1-7. [CrossRef] 
227. Ahuja, D.; Rainu, A.S.; Singh, M.; Kaushik, A. Encapsulation of NPK fertilizer for slow release using sodium carboxymethyl cellulose-g-poly (AA-C0-AM-C0-AMPS)/ Montmorillonite clay-based nanocomposite hydrogels for sustainable agricultural applications. Trends Carbohydr. Res. 2020, 12, 15-23.

228. Lu, Y.; Wu, F.; Duan, W.; Zhou, X.; Kong, W. Engineering a "PEG-g-PEI/DNA nanoparticle-in- PLGA microsphere" hybrid controlled release system to enhance immunogenicity of DNA vaccine. Mater. Sci. Eng. C 2020, 106, 110394. [CrossRef]

229. Grebenik, E.A.; Surin, A.M.; Bardakova, K.N.; Dermina, T.S.; Minaev, N.V.; Veryasova, N.N.; Artyukhova, M.A.; Krasilnikova, I.A.; Bakaeva, Z.V.; Sorokina, E.G.; et al. Chitosan-g-oligo(L,L-lactide) copolymer hydrogel for nervous tissue regeneration in glutamate excitotoxicity: In vitro feasibility evaluation. Biomed. Mater. 2020, 15, 015011. [CrossRef] [PubMed]

230. Barth, H.G.; Jackson, C.; Boyes, B.E. Size Exclusion Chromatography. Anal. Chem. 1994, 66, 595-620. [CrossRef] [PubMed]

231. Hamielec, A.; Gloor, P.; Zhu, S. Kinetics of, free radical modification of polyolefins in extruders-Chain scission, crosslinking and grafting. Can. J. Chem. Eng. 1991, 69, 611-618. [CrossRef]

232. Chaimberg, M.; Cohen, Y. Kinetic Modeling of Free-Radical Graft Polymerization. AIChE J. 1994, 40, 294-311. [CrossRef]

233. Guillot, J.; Leroux, D. Modelling of size-exclusion chromatograms from molecular weight distribution calculations. Application to the grafting of polymers onto functionalized silica. Macromol. Chem. Phys. 1994, 195, 1463-1470. [CrossRef]

234. Hojabr, S.; Baker, W.; Russell, K.; McLellan, P.; Huneault, M. Melt grafting of glycidyl methacrylate onto polyethylene: An experimental and mathematical modeling study. Int. Polym. Proc. 1998, 13, 118-128. [CrossRef]

235. Machado, A.; Gaspar-Cunha, A.; Covas, J. Modelling of the grafting of maleic anhydride onto polyethylene in an extruder. Mater. Sci. Forum 2004, 455-456, 763-766. [CrossRef]

236. Giudici, R. Mathematical modeling of the crafting of maleic anhydride onto polypropylene. Macromol. Symp. 2007, 259, 354-364. [CrossRef]

237. Diaconescu, R.; Grigoriu, A.-M.; Luca, C. Neural network modeling of monochlorotriazinyl- $\beta$-cyclodextrin grafting on cellulosic supports. Cell. Chem. Technol. 2007, 41, 385-390.

238. Luca, C.; Grigoriu, A.-M.; Diaconescu, R.; Secula, M. Modeling and simulation of monochlorotriazinyl- $\beta$-cyclodextrin paper grafting by artificial neural network. Rev. Chim. 2011, 62, 1033-1038.

239. Grigoriu, A.; Racu, C.; Diaconescu, R.; Grigoriu, A.-M. Modeling of the simultaneous process of wet spinning-grafting of bast fibers using artificial neural networks. Textile Res. J. 2012, 82, 324-335. [CrossRef]

240. Tong, G.-S.; Liu, T.; Hu, G.-H.; Hoppe, S.; Zhao, L.; Yuan, W.-K. Modelling of the kinetics of the supercritical CO2 assisted grafting of maleic anhydride onto isotactic polypropylene in the solid state. Chem. Eng. Sci. 2007, 62, 5290-5294. [CrossRef]

241. Wang, J.; Ran, Y.; Ding, L.; Wang, D. Advances in supercritical CO2 assisted grafting of polypropylene in solid state. Chem. React. Eng. Technol. 2008, 24, 173-177.

242. Casis, N.; Estenoz, D.; Vega, J.; Meira, G. Bulk prepolymerization of styrene in the presence of polybutadiene: Determination of grafting efficiency by size exclusion chromatography combined with a new extended model. J. Appl. Polym. Sci. 2009, 111, 1508-1522. [CrossRef]

243. Badel, T.; Beyou, E.; Bounor-Legaré, V.; Chaumont, P.; Flat, J.; Michel, A. Free radical graft copolymerization of methyl methacrylate onto polyolefin backbone: Kinetics modeling through model compounds approach. Macromol. Chem. Phys. 2009, 210, 1087-1095. [CrossRef]

244. Gianoglio Pantano, I.; Asteasuain, M.; Sarmoria, C.; Brandolin, A. Graft copolymers for blend compatibilization. Mathematical modeling of the grafting process. In Proceedings of the 2010 AIChE Annual Meeting Conference Proceedings, Salt Lake City, UT, USA, 7-12 November 2010; pp. 1-14.

245. Gianoglio Pantano, I.; Asteasuain, M.; Sarmoria, C.; Brandolin, A. Graft Copolymers for Blend Compatibilization: Mathematical Modeling of the Grafting Process. Macromol. React. Eng. 2012, 6, 406-418. [CrossRef]

246. Gianoglio Pantano, I.A.; Brandolin, A.; Sarmoria, C. Mathematical modeling of the graft reaction between polystyrene and polyethylene. Polym. Degrad. Stabil. 2011, 96, 416-425. [CrossRef]

247. Aguiar, L.; Pessôa-Filho, P.; Giudici, R. Mathematical modeling of the grafting of maleic anhydride onto poly(propylene): Model considering a heterogeneous medium. Macromol. Theory Simul. 2011, 20, 837-849. [CrossRef]

248. Damodaran, V.; Fee, C.; Popat, K. Modeling of PEG grafting and prediction of interfacial force profile using x-ray photoelectron spectroscopy. Surf. Interface Anal. 2012, 44, 144-149. [CrossRef]

249. Zhou, D.; Gao, X.; Wang, W.-J.; Zhu, S. Termination of surface radicals and kinetic modeling of ATRP grafting from flat surfaces by addition of deactivator. Macromolecules 2012, 45, 1198-1208. [CrossRef]

250. Nasef, M.; Shamsaei, E.; Ghassemi, P.; Aly, A.; Yahaya, A. Modeling, prediction, and multifactorial optimization of radiationinduced grafting of 4-vinylpyridine onto poly(vinylidene fluoride) films using statistical simulator. J. Appl. Polym. Sci. 2013, 127, 1659-1666. [CrossRef]

251. Nasef, M.M.; Ali, A.; Saidi, H.; Ahmad, A. Modeling and optimization aspects of radiation induced grafting of 4-vinylpyridene onto partially fluorinated films. Radiat. Phys. Chem. 2014, 94, 123-128. [CrossRef]

252. Wu, L.L.; Bu, Z.; Gong, C.; Li, B.-G.; Hungenberg, K.-D. Graft Copolymerization of Styrene and Acrylonitrile in the Presence of Poly(propylene glycol): Modeling and Simulation of Semi-Batch and Continuous Processes. Macromol. React. Eng. 2012, 6, 384-394. [CrossRef]

253. Xie, X.-L.; Tong, Z.-F.; Huang, Z.-Q.; Zhang, Y.-Q. Kinetics model of graft co-polymerization of acrylamide onto mechanicallyactivated starch in inverse emulsion. J. Chem. Eng. Chin. Univ. 2014, 28, 567-573. 
254. Liu, X.; Nomura, M. Kinetic modeling and simulation of emulsion grafting copolymerization of styrene and acrylonitrile in the presence of polybutadiene seed latex particles. Ind. Eng. Chem. Res. 2014, 53, 17580-17588. [CrossRef]

255. Zhang, M.; Jia, Y. Kinetic study on free radical grafting of polyethylene with acrylic acid by reactive extrusion. J. Appl. Polym. Sci. 2014, 131, 40990. [CrossRef]

256. Sirirat, T.; Vatanatham, T.; Hansupalak, N.; Rempel, G.; Arayapranee, W. Kinetics and modeling of methyl methacrylate graft copolymerization in the presence of natural rubber latex. Korean J. Chem. Eng. 2015, 32, 980-992. [CrossRef]

257. Oliveira, D.; Dias, R.; Costa, M. Modeling RAFT Gelation and Grafting of Polymer Brushes for the Production of Molecularly Imprinted Functional Particles. Macromol. Symp. 2016, 370, 52-65. [CrossRef]

258. Saeb, M.; Rezaee, B.; Shadman, A.; Formela, K.; Ahmadi, Z.; Hemmati, F.; Kermaniyan, T.; Mohammadi, Y. Controlled grafting of vinylic monomers on polyolefins: A robust mathematical modeling approach. Des. Monomers Polym. 2017, 20, 250-268. [CrossRef]

259. Hernández-Ortiz, J.; Van Steenberge, P.; Reyniers, M.-F.; Marin, G.; D’hooge, D.; Duchateau, J.; Remerie, K.; Toloza, C.; Vaz, A.; Schreurs, F. Modeling the reaction event history and microstructure of individual macrospecies in postpolymerization modification. AIChE J. 2017, 63, 4944-4961. [CrossRef]

260. Hernández-Ortiz, J.; Van Steenberge, P.; Duchateau, J.; Toloza, C.; Schreurs, F.; Reyniers, M.-F.; Marin, G.; D’hooge, D. Sensitivity Analysis of Single-Phase Isothermal Free Radical-Induced Grafting of Polyethylene. Macromol. Theory Simul. 2018, 27, 1800036. [CrossRef]

261. Hernández-Ortiz, J.; Van Steenberge, P.; Duchateau, J.; Toloza, C.; Schreurs, F.; Reyniers, M.-F.; Marin, G.; D’hooge, D. The Relevance of Multi-Injection and Temperature Profiles to Design Multi-Phase Reactive Processing of Polyolefins. Macromol. Theory Simul. 2019, 28, 1900035. [CrossRef]

262. Penlidis, A.; MacGregor, J.F.; Hamielec, A.E. Dynamic modeling of emulsion polymerization reactors. AIChE J. 1985, 31, 881-889. [CrossRef]

263. Iedema, P.D.; Grcev, S.; Hoefsloot, H.C.J. Molecular weight distribution modeling of radical polymerization in a CSTR with long chain branching through transfer to polymer and terminal double bond. Macromolecules 2003, 36, 458-476. [CrossRef]

264. Dias, R.; Costa, M. A new look at kinetic modeling of nonlinear free radical polymerizations with terminal branching and chain transfer to polymer. Macromolecules 2003, 36, 8853-8863. [CrossRef]

265. Krallis, A.; Kiparissides, C. Mathematical modeling of the bivariate molecular weight-Long chain branching distribution of highly branched polymers: A population balance approach. Chem. Eng. Sci. 2007, 62, 5304-5311. [CrossRef]

266. Kryven, I.; Iedema, P.D. A novel approach to population balance modeling of reactive polymer modification leading to branching. Macromol. Theory Simul. 2013, 22, 89-106. [CrossRef]

267. Wang, R.; Luo, Y.; Li, B.-G.; Zhu, S. Modeling of Branching and Gelation in RAFT Copolymerization of Vinyl/Divinyl Systems. Macromolecules 2009, 42, 85-94. [CrossRef]

268. Yaghini, N.; Iedema, P.D. Molecular weight and branching distribution modeling in radical polymerization with transfer to polymer and scission under gel conditions and allowing for multiradicals. Macromolecules 2014, 47, 4851-4863. [CrossRef]

269. Penlidis, A.; Vivaldo-Lima, E.; Hernández-Ortiz, J.; Saldívar-Guerra, E. Chapter 12: Polymer Reaction Engineering. In Handbook of Polymer Synthesis, Characterization, and Processing, 1st ed.; John Wiley \& Sons: New York, NY, USA, 2013; pp. 251-271, ISBN 978-047-063-032-7.

270. Zhu, S.; Hamielec, A. Polymerization kinetic modeling and macromolecular. In Polymer Science: A Comprehensive Reference, 1st ed.; Matyjaszewski, K., Möller, M., Eds.; Elsevier B.V: London, UK, 2012; Chapter 4.32; Volume 4, pp. 779-831, ISBN 978-008-087-862-1.

271. Quintero-Ortega, I.; Vivaldo-Lima, E.; Luna-Bárcenas, G.; Alvarado, J.; Louvier-Hernández, J.; Sanchez, I. Modeling of the Free-Radical Copolymerization Kinetics with Cross Linking of Vinyl/Divinyl Monomers in Supercritical Carbon Dioxide. Ind. Eng. Chem. Res. 2005, 44, 2823-2844. [CrossRef]

272. Dong, P.; Sun, H.; Quan, D. Synthesis of poly(L-lactide-co-5-amino-5-methyl-1,3-dioxan-2-ones) [P(L-LA-co-TAc)] containing amino groups via organocatalysis and post-polymerization functionalization. Polymer 2016, 97, 614-622. [CrossRef] 